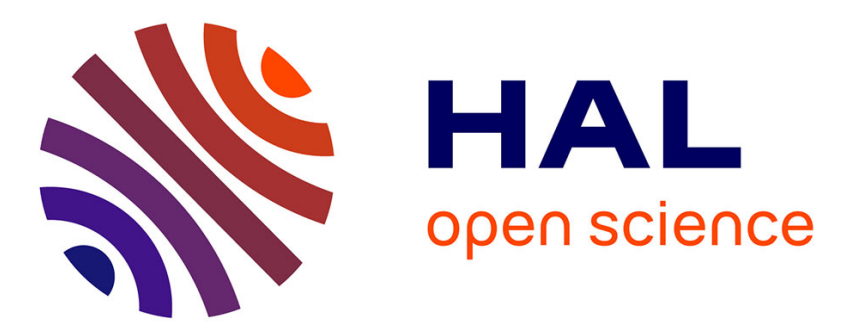

\title{
Stimuler et pérenniser les investissements des jeunes dans l'agriculture et les systèmes alimentaires
}

\author{
Yannick Fiedler
}

\section{To cite this version:}

Yannick Fiedler. Stimuler et pérenniser les investissements des jeunes dans l'agriculture et les systèmes alimentaires: Recommandations politiques basées sur les enseignements tirés de onze pays africains. [Rapport de recherche] Food and Agriculture Organization of the United Nations. 2021, pp.68. hal03386055

\section{HAL Id: hal-03386055 \\ https://hal-sciencespo.archives-ouvertes.fr/hal-03386055}

Submitted on 19 Oct 2021

HAL is a multi-disciplinary open access archive for the deposit and dissemination of scientific research documents, whether they are published or not. The documents may come from teaching and research institutions in France or abroad, or from public or private research centers.
L'archive ouverte pluridisciplinaire HAL, est destinée au dépôt et à la diffusion de documents scientifiques de niveau recherche, publiés ou non, émanant des établissements d'enseignement et de recherche français ou étrangers, des laboratoires publics ou privés. 

pour l'alimentation et l'agriculture

STIMULER ET PÉRENNISER LES

INVESTISSEMENTS DES JEUNES DANS

LAGRICULTURE ET LES SYSTÈMES ALIMENTAIRES

RECOMMANDATIONS POLITIQUES BASÉES SUR LES ENSEIGNEMENTS TIRÉS DE ONZE PAYS AFRICAINS

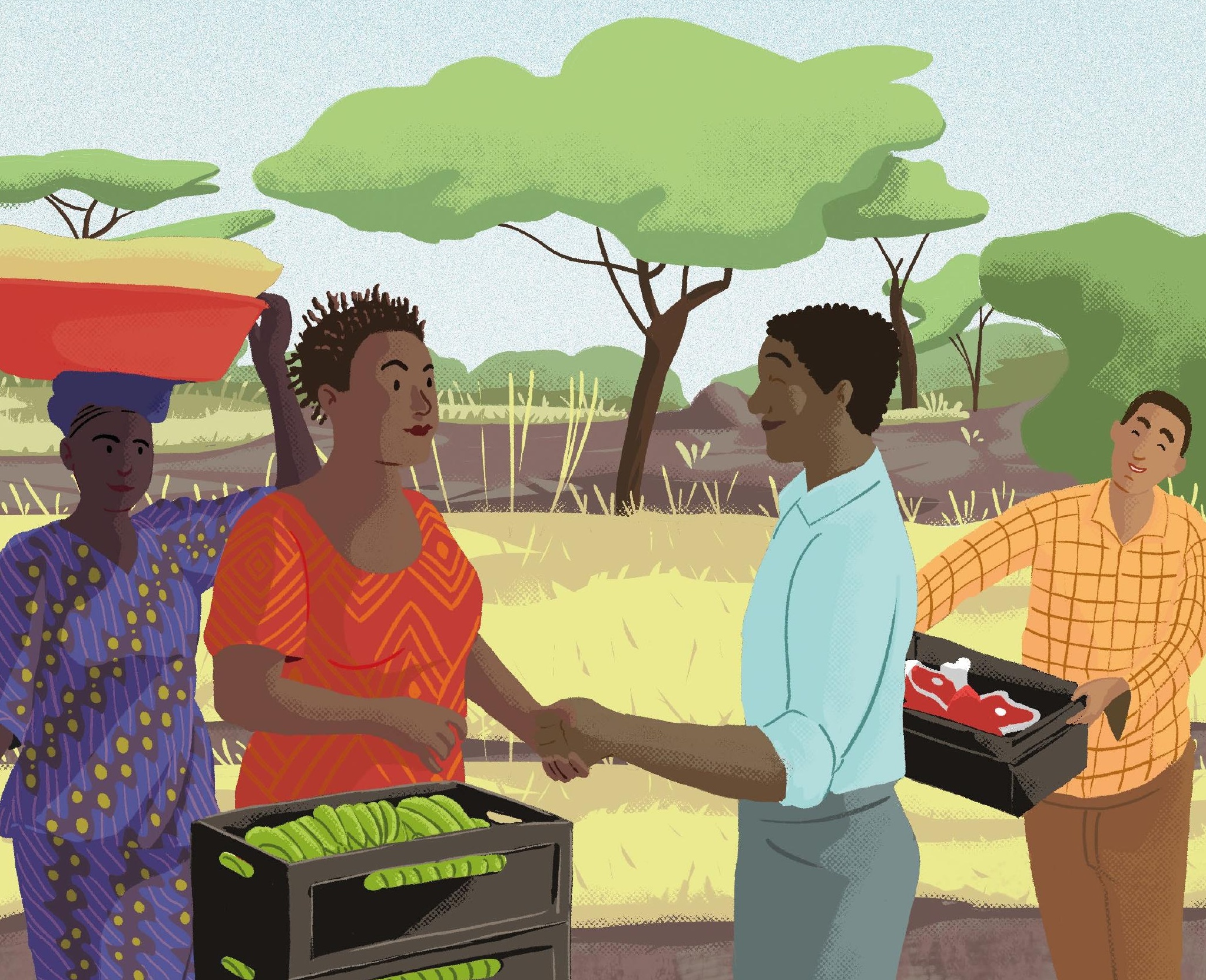





\section{STIMULER ET PÉRENNISER LES INVESTISSEMENTS DES JEUNES DANS LAGRICULTURE ET LES SYSTÈMES ALIMENTAIRES}

RECOMMANDATIONS POLITIQUES BASÉES SUR LES ENSEIGNEMENTS TIRÉS DE ONZE PAYS AFRICAINS

Yannick Fiedler

Organisation des Nations Unies pour l'alimentation et l'agriculture Rome, 2020 
Citer comme suit:

Fiedler, Y. 2020. Stimuler et pérenniser les investissements des jeunes dans l'agriculture et les systèmes alimentaires - Recommandations politiques basées sur les enseignements tirés de onze pays africains. Rome, FAO. https://doi.org/10.4060/cb1124fr

Les appellations employées dans ce produit d'information et la présentation des données qui y figurent n'impliquent de la part de l'Organisation des Nations Unies pour l'alimentation et l'agriculture (FAO) aucune prise de position quant au statut juridique ou au stade de développement des pays, territoires, villes ou zones ou de leurs autorités, ni quant au tracé de leurs frontières ou limites. Le fait qu'une société ou qu'un produit manufacturé, breveté ou non, soit mentionné ne signifie pas que la FAO approuve ou recommande ladite société ou ledit produit de préférence à d'autres sociétés ou produits analogues qui ne sont pas cités.

Les opinions exprimées dans ce produit d'information sont celles du/des auteur(s) et ne reflètent pas nécessairement les vues ou les politiques de la FAO.

ISBN 978-92-5-133653-3

(C) FAO, 2020

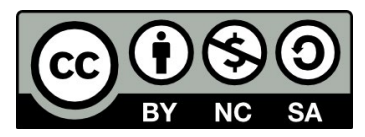

Certains droits réservés. Cette œuvre est mise à la disposition du public selon les termes de la Licence Creative Commons AttributionPas d'Utilisation Commerciale-Partage dans les Mêmes Conditions 3.0 Organisations Intergouvernementales (CC BYNCSA 3.0 IGO; https://creativecommons.org/licenses/by-nc-sa/3.0/igo/legalcode.fr).

Selon les termes de cette licence, cette œuvre peut être copiée, diffusée et adaptée à des fins non commerciales, sous réserve que la source soit mentionnée. Lorsque l'œurre est utilisée, rien ne doit laisser entendre que la FAO cautionne tels ou tels organisation, produit ou service. L'utilisation du logo de la FAO n'est pas autorisée. Si l'œuvre est adaptée, le produit de cette adaptation doit être diffusé sous la même licence Creative Commons ou sous une licence équivalente. Si l'œuvre est traduite, la traduction doit obligatoirement être accompagnée de la mention de la source ainsi que de la clause de non-responsabilité suivante: «La traduction n'a pas été réalisée par I'Organisation des Nations Unies pour l'alimentation et l'agriculture (FAO). La FAO n'est pas responsable du contenu ni de l'exactitude de la traduction. L'édition originale [langue] est celle qui fait foi.»

Tout litige relatif à la présente licence ne pouvant être résolu à l'amiable sera réglé par voie de médiation et d'arbitrage tel que décrit à l'Article 8 de la licence, sauf indication contraire contenue dans le présent document. Les règles de médiation applicables seront celles de l'Organisation mondiale de la propriété intellectuelle (http://www.wipo.int/amc/fr/mediation/rules) et tout arbitrage sera mené conformément au Règlement d'arbitrage de la Commission des Nations Unies pour le droit commercial international (CNUDCI).

Matériel attribué à des tiers. II incombe aux utilisateurs souhaitant réutiliser des informations ou autres éléments contenus dans cette œuvre qui y sont attribués à un tiers, tels que des tableaux, des figures ou des images, de déterminer si une autorisation est requise pour leur réutilisation et d'obtenir le cas échéant la permission de l'ayant-droit. Toute action qui serait engagée à la suite d'une utilisation non autorisée d'un élément de l'œuvre sur lequel une tierce partie détient des droits ne pourrait l'être qu'à l'encontre de l'utilisateur.

Ventes, droits et licences. Les produits d'information de la FAO sont disponibles sur le site web de la FAO (www.fao.org/publications) et peuvent être obtenus sur demande adressée par courriel à: publications-sales@fao.org. Les demandes visant un usage commercial doivent être soumises à: www.fao.org/contact-us/licence-request. Les questions relatives aux droits et aux licences doivent être adressées à: copyright@fao.org. 


\section{TABLE DES MATIÈRES}

Avant-propos vii

- Office fédéral suisse de l'agriculture vii

- Organisation des Nations Unies pour l'alimentation et l'agriculture viii

$\begin{array}{ll}\text { Remerciements } & \text { ix }\end{array}$

Abréviations $\quad$ xi

Résumé

$\begin{array}{ll}\text { Introduction } & 1\end{array}$

$\begin{array}{ll}\text { I. MÉTHODOLOGIE } & 7\end{array}$

II. UN APERÇU SOCIO-ÉCONOMIQUE DE L'ÉTAT DE LA JEUNESSE ET 11 DE LAGRICULTURE

1. Agriculture et productivité agricole 11

2. Éducation des jeunes et chômage des jeunes 14

III. PERMETTRE AUX JEUNES D'ACCÉDER AUX FACTEURS DE 17 PRODUCTION ET AUX SERVICES D'APPUI TECHNIQUE

$\begin{array}{ll}\text { 1. Accès aux services financiers } & 17\end{array}$

1.1. Accès aux services financiers commerciaux et informels $\quad 17$

1.2. Incitations financières: défis, opportunités et bonnes pratiques 20

1.3. Recommandations politiques: améliorer l'accès aux services financiers 25

2. Accès à la terre $\quad 27$

2.1. Accès des jeunes à la terre par le biais d'un l'héritage, du marché et de 28 programmes de distribution

2.2. Recommandations politiques: améliorer l'accès à la terre 
3. Information et services techniques 31

3.1. Accès aux incubateurs, aux services d'encadrement et de conseil 32

3.2. Recommandations politiques: améliorer l'accès aux services techniques et 33 d'information

IV. IMPLIQUER LES JEUNES DANS LA CONCEPTION DES POLITIQUES, DES LOIS ET DES MESURES D'INCITATION

1. Participation des jeunes aux processus décisionnels: analyse de la situation 36

2. Recommandations: permettre aux jeunes de participer à l'élaboration 38 des politiques

V. CINQ RECOMMANDATIONS POLITIQUES DANS LE BUT DE PERMETTRE AUX JEUNES ENTREPRENEURS AGRICOLES D'INVESTIR DANS L'AGRICULTURE ET LES SYSTÈMES ALIMENTAIRES

Remarques finales et perspectives

Références 


\section{Figures:}

- Figure 1: Valeur ajoutée brute de l'agriculture (\% du PIB), emploi dans l'agriculture (\% de l'emploi total) et valeur ajoutée agricole par travailleur (en USD) en Guinée, au Malawi, au Mali, au Mozambique et en Ouganda

- Figure 2: Valeur ajoutée brute de l'agriculture (\% du PIB), emploi dans l'agriculture (\% de l'emploi total) et valeur ajoutée agricole par travailleur (en USD) en Côte d'Ivoire, en Mauritanie et au Sénégal

- Figure 3: Valeur ajoutée brute de l'agriculture (\% du PIB), emploi dans l'agriculture (\% de l'emploi total) et valeur ajoutée agricole par travailleur (en USD) en Namibie, en Afrique du Sud et en Tunisie

- Figure 4: Éducation - taux de scolarisation par pays

- Figure 5: Emploi vulnérable, sous-emploi et chômage des jeunes

- Figure 6: Jeunes qui déclarent avoir des comptes bancaires (\% du total des jeunes)

- Figure 7: Accès des jeunes agri-entrepreneurs et des jeunes agriculteurs aux services financiers, comme indiqué dans les évaluations des capacités ( 0 = pas d'accès, $5=$ excellent accès)

- Figure 8: Crédit à l'agriculture (\% du total des crédits) et valeur ajoutée brute de l'agriculture (\% du PIB)

- Figure 9: Accès des jeunes à la terre tel qu'identifié dans les processus d'évaluation et de planification ( $0=$ pas d'accès; $5=$ excellent accès)

- Figure 10: Accès à l'information et aux services techniques tels qu'identifiés dans les processus d'évaluation et de planification $(0=$ pas d'accès; $5=$ excellent accès)

- Figure 11: Participation des jeunes aux mécanismes de coordination/ plateformes multi-acteurs $(0=$ pas de participation; $5=$ excellente participation)

\section{Encadrés:}

- Encadré 1: Que sont les incitations à l'investissement? 5

- Encadré 2: Les prêts fonciers tunisiens 22

- Encadré 3: Le modèle économique de I'Union nationale des mutuelles 24 d'investissement et de crédit oasien et des zones pluviales (UNMICO) 



\section{Avant-propos}

\section{Office fédéral suisse de l'agriculture}

La mise en place de systèmes alimentaires durables est essentielle à la réalisation du Programme de développement durable à I'horizon 2030, le principal cadre international pour rendre ce monde meilleur. Cela exige de modifier radicalement la façon dont nous produisons et consommons les aliments. La Suisse est convaincue que ce n'est que si nous réussissons à impliquer les jeunes en tant que professionnels des systèmes alimentaires que cette transformation sera couronnée de succès.

Pourtant, dans le monde, peu de jeunes entrevoient un avenir dans l'agriculture ou les zones rurales. L'âge moyen des agriculteurs augmente et il y a peu de chances que les jeunes générations remplacent les agriculteurs vieillissants, ce qui entraîne ce que l'on appelle un «écart de génération» dans les secteurs agricole et agro-alimentaire.

Les jeunes hésitent d'une part à considérer l'agriculture comme une option de subsistance viable et l'associent d'autre part à de faibles rendements, un travail acharné et un statut social bas. D'autre part, les jeunes entrepreneurs souhaitant réussir dans les filières agricoles et agro-alimentaires sont confrontés à de nombreuses difficultés, notamment à un accès insuffisant aux terres, au crédit et aux marchés. Ces obstacles sont multidimensionnels et nécessitent des interventions à différents niveaux.

En soutenant le programme-cadre FAO RAI, la Suisse contribue à la recherche d'investissements responsables nécessaires pour attirer et garantir le succès des jeunes dans les systèmes agricoles et alimentaires. Avec un financement suisse, la FAO a mis au point un outil pour identifier les capacités existantes et nécessaires qui permettront aux jeunes de réaliser et de bénéficier de ces investissements.

À la demande de la FAO, le Comité national suisse de la FAO a examiné cet outil. Le Comité est un organe consultatif du gouvernement fédéral suisse pour les questions relatives à la sécurité alimentaire et aux systèmes alimentaires durables. La version révisée de l'outil a été testée en Tunisie. Les résultats ont été traduits en une vision stratégique et un ensemble d'interventions concrètes visant à créer un environnement propice à l'investissement pour les jeunes agri-entrepreneurs dans d'autres contextes et pays.

L'Office fédéral de l'agriculture (OFAG) tient à exprimer sa profonde gratitude à la FAO pour son dévouement et ses efforts continus visant à promouvoir et à soutenir la participation des jeunes à l'agriculture et aux systèmes alimentaires.

La jeunesse est l'avenir de la sécurité alimentaire. Le rôle de la jeunesse en tant qu'agent de la transformation de l'agriculture et des systèmes alimentaires doit attirer une attention accrue au niveau mondial pour induire un changement positif tout au long du Programme 2030.

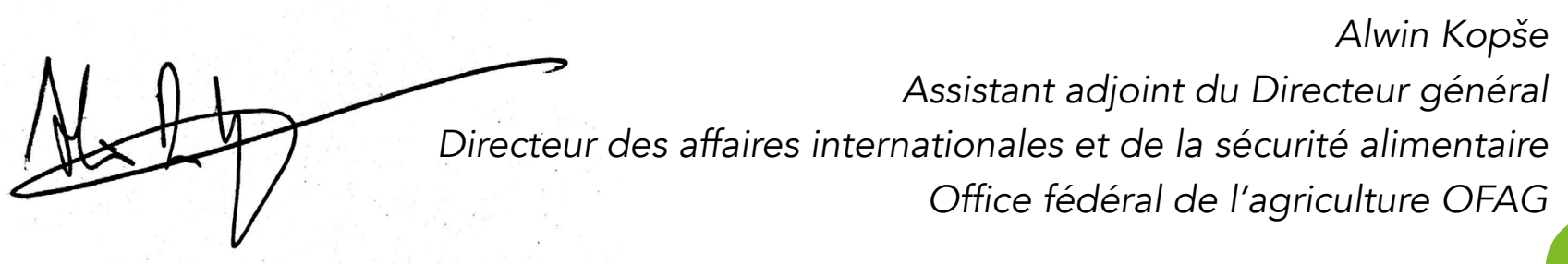




\section{Avant-propos}

\section{L'Organisation des Nations Unies pour l'alimentation et l'agriculture}

Investir dans la jeunesse et donner aux jeunes agri-entrepreneurs les moyens d'investir de manière responsable dans leurs fermes et entreprises sont des actions fondamentales pour atteindre de nombreux Objectifs de développement durable. Les jeunes sont l'avenir de la sécurité alimentaire pour tous, mais de nombreux jeunes n'entrevoient aucun avenir pour eux-mêmes dans l'agriculture en raison des nombreux obstacles auxquels ils sont confrontés. Les taux élevés de chômage des jeunes, en particulier dans les zones rurales, exigent des réponses politiques déterminées pour stimuler une croissance économique inclusive. Une action concertée est maintenant plus urgente que jamais, car les impacts socio-économiques de la pandémie de COVID-19 ont encore accru la vulnérabilité des jeunes femmes et hommes du monde entier.

Avec le soutien de la Suisse, la FAO a mis au point des outils pratiques d'évaluation des capacités et de planification stratégique pour aider les pays à déterminer les principales lacunes en matière de politiques et de capacités, ainsi que des solutions pratiques pour donner aux jeunes les moyens d'investir dans l'agriculture et les systèmes alimentaires et d'assurer la durabilité de leurs investissements. Ces outils ont été utilisés dans onze pays africains entre 2017 et 2020 à travers des processus participatifs qui ont permis aux jeunes de s'exprimer sur les sujets qui les concernent le plus. Des recommandations pratiques et des plans d'action ont été rédigés afin que chaque pays puisse s'en servir pour orienter les processus politiques au niveau national.

Ce rapport présente les principales conclusions et leçons apprises, ainsi que les principales recommandations politiques issues des preuves empiriques recueillies. Il souligne l'importance d'institutionnaliser les besoins spécifiques des jeunes et d'intégrer des mécanismes spécifiques aux jeunes dans les politiques de promotion des investissements et les plans d'incitation pour rendre les investissements dans le secteur agroalimentaire plus attractifs et viables pour les jeunes femmes et hommes. Plus important encore, il souligne qu'une participation effective des jeunes au dialogue politique est une condition préalable à des politiques efficaces et fructueuses de promotion des investissements centrées sur les jeunes.

Je souhaite exprimer ma sincère gratitude à l'Office fédéral suisse de l'agriculture pour son soutien continu et son engagement constant à travailler ensemble pour relever deux des défis les plus pressants de notre époque: assurer une sécurité alimentaire durable tout en créant des opportunités pour les jeunes dans le secteur agroalimentaire. Je voudrais également remercier nos institutions partenaires, nos collègues de la FAO et les experts indépendants qui ont travaillé conjointement pour faire de cet effort de collaboration un succès.

Marcela Villarreal, PhD

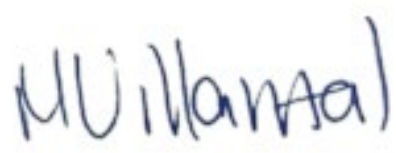

Directrice, Partenariats et collaboration au sein du système des Nations Unies

Organisation des Nations Unies pour l'alimentation et l'agriculture 


\section{Remerciements}

Ce rapport a été rédigé par Yannick Fiedler, Chargé de programme de la FAO. II résume les principales conclusions et leçons tirées des travaux de la FAO visant à donner aux jeunes agri-entrepreneurs les moyens d'investir dans l'agriculture et les systèmes alimentaires avec onze pays africains. II s'appuie donc sur un ensemble de preuves empiriques qui n'auraient pas pu être produites sans le soutien inestimable de nombreuses institutions et individus.

Le soutien de l'Office fédéral suisse de l'agriculture (OFAG) a été essentiel pour lancer et étendre ces travaux. Depuis 2017, I'OFAG a apporté d'importantes contributions financières et techniques qui ont permis à la FAO de créer et de déployer des outils innovants d'évaluation et de planification stratégique. Le soutien et les commentaires continus d'Alwin Kopše, Madeleine Kaufmann et Kate Dassesse ont contribué incontestablement à affiner nos propres approches.

L'auteur tient également à exprimer sa sincère gratitude aux nombreux chercheurs qui se sont engagés dans les processus d'évaluation et qui ont rédigé d'excellents rapports et documents d'information. En particulier, l'auteur voudrait reconnaître spécifiquement les contributions inestimables de Mohamed Elloumi, Abdallah Ben Saad, Emna Ouertani et Ahmed Yangui de l'Institut National de la Recherche Agronomique de Tunisie (INRAT). Ces participants ont mené à bien le processus global de planification stratégique en Tunisie, affiné les outils d'évaluation de la FAO, et ont permis à l'auteur de mieux comprendre les défis et opportunités liés à l'autonomisation des jeunes de manière plus générale. En outre, les contributions importantes des experts en la matière qui ont préparé des documents d'information et participé aux évaluations des capacités sont fortement appréciées: Elina Amadhila (Namibie), Kama Berté (Côte d'Ivoire), Cyriaque Hakizimana (Afrique du Sud), Stephen Lwasa (Ouganda), Joao Mutondo (Mozambique) and Kumbukani Ng'ambi (Malawi).

D'autre part, la grande qualité des évaluations qui ont alimenté ce rapport n'aurait pas pu être atteinte sans l'engagement ferme des bureaux de pays et régionaux de la FAO et des participants aux différents ateliers, notamment des représentants des organisations de jeunesse, du gouvernement, de la société civile, du secteur privé et des institutions financières, ainsi que des deux institutions partenaires qui ont participé aux évaluations en Tunisie (Haute école spécialisée bernoise) et en Afrique australe (Centre d'information, de formation et de sensibilisation pour l'Afrique). Leur temps, leurs efforts et leur volonté de contribuer à l'identification des difficultés et des opportunités auxquels font face les jeunes agri-entrepreneurs sont chaleureusement reconnus.

Enfin, I'auteur tient à exprimer sa plus sincère gratitude aux collègues suivants qui ont contribué à ce rapport: Michael Riggs, chef d'équipe RAl, a fourni ses conseils et ses commentaires tout au long du processus. Jana Herold, Emma McGhie et Tony Nsanganira de la FAO, Alwin Kopše et Kate Dassesse de l'OFAG, Mohamed Elloumi de I'INRAT, Barbara Eiselen de la HAFL et Mercy Moyo de l'ITOCA ont examiné différentes versions de ce rapport et ont fourni des commentaires utiles. La mise en page et la conception graphique ont été effectuées par Carolina Saiz, et la traduction en langue française par Elizabeth Frolet. 



\section{Abréviations}

AgriBEE Agricultural Black Economic Empowerment

ANPEJ Agence nationale pour la promotion de l'emploi des jeunes

APIA Agence de promotion des investissements agricoles de Tunisie

AsiaDHRRA Asian Partnership for the Development of Human Resources in Rural Asia (Partenariat asiatique pour le développement des ressources humaines en Asie rurale)

BTS Banque tunisienne de solidarité

- CAYC Commercial Agricultural Youth Chamber of South Africa (Chambre commerciale de la jeunesse agricole d'Afrique du Sud)

CFS-RAI (selon son acronyme anglais) Principes du Comité de la sécurité alimentaire mondiale pour un investissement responsable dans l'agriculture et les systèmes alimentaires

CNOP-Mali Coordination nationale des Organisations paysannes du Mali

CNUCED Conférence des Nations Unies sur le commerce et le développement

- CPGD Centre for Research on Governance and Development (Centre pour la recherche sur la gouvernance et le développement)

CTA Centre technique pour la coopération agricole et rurale

- DBN Development Bank of Namibia (Banque de développement de la Namibie)

DT Dinar tunisien

FAIJ Support for Youth Initiatives Fund of Mozambique (Fonds pour le soutien des initiatives de la jeunesse)

FAO Organisation des Nations Unies pour l'alimentation et l'agriculture

FENAJER Fédération nationale des jeunes ruraux du Mali

- FENOPJERCI Fédération nationale des organisations professionnelles de jeunesse rurale de Côte d'Ivoire (National Federation of Professional Rural Youth Organizations of Côte d'Ivoire)

FIDA Fonds international de développement agricole

- FONGIP Fonds de Garantie des Investissements Prioritaires (Senegalese Guarantee Fund for Investments in Priority Sectors) 
- GIZ

INRAT

- MAFISA

MIJA

- NDI

- NYCS

ODD

- OFAG

- OMS

- ONG

- ONU

- PAM

- PIB

- PME

- RAPEA

- R-CAT

- TPE-PME

UNICEF

- UNMICO

- USD

VAB

- YARD
Deutsche Gesellschaft für Internationale Zusammenarbeit (Agence allemande de coopération internationale)

Institut national de la recherche agronomique de Tunisie

Micro Agricultural Financial Institutions of South Africa (micro institutions financières dans le domaine agricole en Afrique du Sud)

Modèle d'insertion des jeunes dans l'agriculture

National Democratic Institute (Institut démocratique national)

Namibian Youth Credit Scheme (Programme namibien de crédit destiné aux jeunes)

Objectif de développement durable

Office fédéral suisse pour l'agriculture

Organisation mondiale de la santé

Organisation non gouvernementale

Organisation des Nations Unies

Programme alimentaire mondial

Produit intérieur brut

Petites et moyennes entreprises

Réseau africain pour la promotion de l'entrepreneuriat agricole

Outil d'évaluation rapide des capacités

Très petites, petites et moyennes entreprises

Fonds des Nations Unies pour l'enfance

Union nationale des mutuelles d'investissement et de crédit oasien et des zones pluviales

Dollar des États-Unis

Valeur ajoutée brute

Youth in Agriculture and Rural Development (les jeunes dans l'agriculture et le développement rural/Afrique du Sud) 


\section{Résumé}

La réalisation des Objectifs de développement durable (ODD) nécessite des efforts importants pour mobiliser des financements et des investissements dans des secteurs qui contribuent à favoriser le développement durable pour les personnes, la planète et la prospérité. Le secteur agroalimentaire est reconnu comme l'un des dix secteurs $d^{\prime}$ investissement prioritaires des ODD, en raison de son fort potentiel à contribuer à l'éradication de la pauvreté et de la faim, ainsi qu'à la création d'une croissance durable et d'emplois décents.

Le secteur privé devrait fournir l'essentiel des investissements nécessaires pour atteindre les ODD. Cependant, des interventions stratégiquement ciblées du secteur public seront essentielles pour stimuler une croissance durable générée, à son tour, par les investissements du secteur privé. Les investissements publics, les stratégies de promotion des investissements et un environnement général favorable sont des catalyseurs des investissements privés alignés sur les stratégies nationales de développement.

À cet égard, il est de plus en plus reconnu que les mesures qui autonomisent les jeunes agri-entrepreneurs devraient être un élément clé d'une telle stratégie de promotion des investissements centrée sur le développement durable. La réalisation même de la sécurité alimentaire des générations futures, la transformation durable des systèmes alimentaires et la lutte contre le chômage et les migrations de détresse dépendent toutes de la mise en œuvre réussie de stratégies qui rendent le secteur agroalimentaire plus attractif pour les jeunes. Cela, à son tour, nécessite des réponses politiques intelligentes qui aideront les jeunes investisseurs à surmonter les nombreux obstacles auxquels ils sont confrontés - le manque d'accès au financement, à la terre, à l'information et aux services techniques - pour ne citer que les plus cruciaux.

Depuis 2017, c'est en effectuant des analyses de capacités participatives et des processus de planification stratégique avec et pour les jeunes que la FAO a fourni un soutien à l'Afrique et à l'Asie du Sud-Est dans le but d'identifier les principales difficultés et les bonnes pratiques qui se présentent aux jeunes agri-entrepreneurs. Ce rapport résume les principales conclusions et leçons tirées du travail de la FAO avec onze pays africains Côte d'Ivoire, Guinée Conakry, Malawi, Mali, Mauritanie, Mozambique, Namibie, Sénégal, Afrique du Sud, Tunisie et Ouganda — en ayant recours à la Boîte à outils de planification stratégique «Permettre aux jeunes agri-entrepreneurs d'investir dans l'agriculture et les systèmes alimentaires».

Ce rapport a pour objet de présenter une vue d'ensemble des principaux défis politiques et des bonnes pratiques qui ont été identifiés dans ces évaluations, ainsi que des recommandations générales pour les décideurs. Tout d'abord, il présente les principaux indicateurs socio-économiques des onze pays africains et propose un regroupement en différents groupes. Ensuite, il examine les défis politiques et les bonnes pratiques qui contribueront à faciliter l'accès des jeunes au financement, à la terre, aux services et informations techniques. Finalement, il analyse l'engagement actuel des organisations de 
jeunes agri-entrepreneurs dans les processus d'élaboration des politiques. Et pour terminer, le rapport propose cinq recommandations clés pour les décideurs.

Le corpus de données empiriques généré par ce travail suggère que le renforcement des mesures d'incitation à l'investissement qui autonomisent spécifiquement les jeunes agrientrepreneurs est essentiel pour accroître l'attractivité du secteur agroalimentaire pour les jeunes et pour stimuler leurs investissements. Ces mesures de soutien comprennent des incitations financières, notamment des programmes de prêts concessionnels et non concessionnels ainsi que des garanties de prêts, des informations et services techniques et des programmes de distribution des terres. Ces incitations ne seront cependant efficaces que si la politique générale et les cadres juridiques autonomisent les jeunes agri-entrepreneurs, au lieu de les désavantager par manque d'attention. À son tour, tant l'efficacité des incitations que la création d'un tel environnement favorable aux jeunes agrientrepreneurs dépendent de l'implication active et de la participation des organisations de jeunes agri-entrepreneurs aux processus d'élaboration des politiques.

Alors que des efforts importants ont été consentis et que des ressources ont été mobilisées dans un ou plusieurs de ces domaines par les onze pays sur lesquels se base cette étude, le rapport conclut qu'il serait souhaitable de se concentrer davantage sur les jeunes dans la promotion des investissements agricoles et qu'en outre, les obstacles qui peuvent compromettre l'efficacité des programmes d'incitation existants demeurent.

En particulier, le rapport fournit aux décideurs politiques les cinq recommandations clés suivantes qui contribueront à responsabiliser les jeunes agri-entrepreneurs: (i) Élaborer des critères de ciblage clairs qui déterminent les conditions d'accès aux programmes $d^{\prime}$ incitation pour garantir que ces appuis correspondent aux besoins de développement national et maximisent le rendement sur l'investissement, tant pour les jeunes bénéficiaires que pour les agences gouvernementales. (ii) Fournir des ensembles d'incitations plutôt que des interventions de soutien autonomes ou isolées. En combinant des prêts avec des subventions d'investissement, des services d'incubation, d'encadrement et de mentorat, les chances d'avoir un retour sur investissement durable dans la jeunesse peuvent être beaucoup plus élevées qu'à travers des programmes isolés et des efforts dispersés. iii) Veiller à ce que la politique générale et le cadre juridique renforcent les investissements des jeunes agri-entrepreneurs au lieu de les entraver. Même lorsque des régimes d'incitation cohérents et bien structurés sont en place, ceux-ci ne sont efficaces que si la politique générale et le cadre juridique autonomisent les jeunes agri-entrepreneurs. (iv) Mettre au point une stratégie de communication adaptée aux jeunes et au contexte qui cherche à atteindre les jeunes en utilisant leurs moyens de communication préférés, tels que les réseaux sociaux, les plates-formes Web, la télévision ou les radios rurales. (v) Faire participer les jeunes aux processus d'élaboration des politiques, à la fois en les impliquant activement dans les consultations multipartites et le dialogue politique, et en envisageant de soutenir une partie tierce neutre pour renforcer leurs capacités là où les organisations de jeunesse ne sont pas encore bien développées. 


\section{Introduction}

\section{Nous devons augmenter considérablement les investissements dans l'agriculture et le développement rural pour atteindre les Objectifs de développement durable.}

La réalisation des Objectifs de développement durable (ODD) nécessite une augmentation significative des investissements. Selon la Conférence des Nations Unies sur le commerce et le développement (CNUCED), au niveau d'investissement de 2014, les pays en développement sont confrontés à un déficit d'investissement annuel d'environ 2,5 billions d'USD (CNUCED, 2014). II est particulièrement urgent d'augmenter les investissements dans les secteurs qui contribuent à l'éradication de la pauvreté et de la faim - qui constituent les deux premiers ODD. Selon les données disponibles les plus récentes, plus de 730 millions de personnes vivent dans l'extrême pauvreté (Banque mondiale, 2020a) et 690 millions de personnes sont toujours touchées par la faim chronique (FAO et al., 2020). La pandémie de COVID-19 et les mesures d'endiguement connexes sont susceptibles d'exacerber davantage les souffrances de ceux qui souffrent déjà. La Banque mondiale estime que 71 à 100 millions de personnes supplémentaires seront poussées dans l'extrême pauvreté (Banque mondiale, 2020b). La FAO, le FIDA, I'UNICEF, le PAM et l'OMS prévoient que 83 à 132 millions de personnes supplémentaires seront sous-alimentées en 2020 en raison des impacts économiques de la pandémie (FAO et al., 2020).

Le secteur agroalimentaire a un potentiel particulièrementélevé pour contribuer à la réduction de la pauvreté et de la faim. Il est donc reconnu comme l'un des dix secteurs d'investissement prioritaires des ODD (CNUCED, 2020). En effet, c'est en raison du lien entre l'agriculture, le développement rural et la réduction de la pauvreté que l'investissement dans l'agriculture est non seulement essentiel pour éradiquer la faim, mais qu'il est également beaucoup plus efficace pour réduire la pauvreté que l'investissement dans tout autre secteur (FAO, 2017). Ce potentiel de transformation du secteur agroalimentaire s'explique par deux faits principaux. Premièrement, la pauvreté reste un phénomène essentiellement rural: à ce jour, environ 80 \% des personnes extrêmement pauvres vivent en zone rurale (De La O Campos et al., 2018). Les investissements dans l'agriculture et les systèmes alimentaires peuvent créer des emplois décents dans les zones rurales, ce qui contribuera à sortir les communautés rurales de la pauvreté. Deuxièmement, les investissements productifs le long des filières agricoles augmenteront la disponibilité d'aliments abordables et nutritifs pour les pauvres des zones urbaines et rurales et réduiront donc la part des dépenses alimentaires totales des ménages.

Malgré ce potentiel, le secteur agricole souffre d'un sous-investissement important et chronique. Des données récentes indiquent que la part de l'agriculture dans les crédits commerciaux et les investissements directs étrangers reste inférieure à 3 pour cent (FAO, 2018a; Fiedler et lafrate, 2016), malgré la contribution indéniable du secteur à l'emploi global et au PIB des pays à revenu faible et intermédiaire. En 2015, la FAO a identifié un 
déficit d'investissement annuel de 265 milliards USD pour atteindre les deux premiers ODD d'ici 2030, dont plus de $50 \%$ devraient cibler l'agriculture et le développement rural (Bellu, Mueller et Kavallari, 2015; un chiffre similaire est estimé par le CNUCED, 2020). Depuis lors, le déficit d'investissement s'est probablement creusé considérablement. En premier lieu, il existe des preuves significatives et inquiétantes d'une nouvelle baisse de certaines formes $d^{\prime}$ investissement dans l'agriculture ${ }^{1}$ au cours des dernières années. En second lieu, il est prévu que les investissements dans le secteur agroalimentaire continueront à diminuer en raison des impacts du "grand confinement» induit par la COVID-19, qui ont provoqué une chute des prix alimentaires mondiaux et des perspectives limitées à court terme de demande croissante d'aliments de meilleure qualité dans les marchés émergents (Schmidhuber et Qiao, 2020). Par conséquent, augmenter les investissements dans l'agriculture et les systèmes alimentaires est plus urgent que jamais.

L'essentiel des investissements nécessaires pour atteindre les ODD devrait provenir du secteur privé. Les agriculteurs et les très petites, petites et moyennes entreprises (TPE-PME), qui sont les plus gros investisseurs dans l'agriculture des pays en développement (FAO, 2012a), joueront un rôle clé à cet égard. Cependant, des interventions stratégiquement ciblées du secteur public seront essentielles pour stimuler une croissance durable mûe par le secteur privé. Les investissements publics, les stratégies de promotion des investissements et un environnement général favorable sont des catalyseurs des investissements privés qui devraient être alignés sur les stratégies nationales de développement.

\section{Permettre aux jeunes d'investir le long des chaînes de valeur agricoles devrait être une composante essentielle d'un régime de promotion des investissements axé sur le développement durable.}

Les mesures qui permettent aux jeunes - notamment aux jeunes agriculteurs familiaux et aux agri-entrepreneurs - d'investir dans leurs propres fermes et entreprises le long des filières agricoles devraient être un élément clé d'une stratégie gouvernementale axée sur le développement durable visant à renforcer les investissements au niveau national. II existe des preuves significatives que l'autonomisation des jeunes est essentielle pour parvenir à une sécurité alimentaire durable, améliorer la productivité durable, la valeur ajoutée et la résilience, et pour lutter contre le chômage, les migrations de détresse et la pauvreté.

Premièrement, la production alimentaire mondiale devra augmenter de manière significative pour satisfaire la demande supplémentaire générée par la croissance démographique continue (Alexandratos et Bruinsma, 2012). Les petits producteurs, aussi bien les agriculteurs que les transformateurs après récolte, représentent une part importante de la nourriture mondiale consommée - jusqu'à 80 pour cent en Asie et en Afrique subsaharienne (FIDA, 2011). Cependant, le «fossé générationnel» agricole - le vieillissement de la maind'œuvre agricole et le non-rajeunissement de tout un secteur — risque de compromettre

\footnotetext{
1 Le Rapport sur Les investissements dans le monde 2020 de la CNUCED montre par exemple que par rapport à la période 2010-2014, l'investissement étranger moyen annuel dans l'agriculture a diminué de $28 \%$ pendant la période 2015-2018, malgré des perspectives macroéconomiques plus favorables (CNUCED, 2020, p. 185 ).
} 
la réalisation d'une sécurité alimentaire durable 2 . Selon une étude récente, l'âge moyen d'un agriculteur africain est d'environ 60 ans (FAO, 2014; voir aussi: Rapsomanikis, 2015), ce qui peut, dans un avenir prévisible, entraîner une pénurie d'agriculteurs (YPARD, 2017). Combler le fossé des générations agricoles nécessite des politiques et des stratégies qui offrent des opportunités et des perspectives aux jeunes qui souhaitent s'engager dans l'agriculture.

Deuxièmement, les jeunes peuvent être des agents du changement dans la transformation des systèmes agroalimentaires. Les systèmes de production agricole doivent devenir plus efficaces, durables et résilients. La vulnérabilité des communautés et des filières agricoles à la suite des mesures d'endiguement de la COVID-19 et leurs effets socio-économiques ont mis en évidence la nécessité de systèmes alimentaires plus résilients et robustes (FAO, 2020). Dans le même temps, trop de petits agriculteurs et transformateurs opèrent encore à des niveaux de subsistance ou de quasi-subsistance, et beaucoup d'autres font face à des revenus inférieurs en raison de l'évolution de la situation du marché. En exploitant leur potentiel d'innovation, en utilisant de nouvelles technologies et techniques et en tirant parti de nouvelles opportunités dans les chaînes de valeur émergentes, les jeunes agrientrepreneurs pourraient créer des entreprises prospères et améliorer la productivité et la valeur ajoutée de la production agricole. À un autre niveau, renforcer la participation des jeunes à l'agriculture pourrait accroître la production alimentaire respectueuse de l'environnement, car les jeunes entrepreneurs sont à l'origine d'innovations dans l'économie verte (OIT, 2017). Pour ces raisons, un consensus émerge selon lequel l'augmentation de la productivité des jeunes engagés dans les chaînes de valeur agricoles n'augmente pas seulement leur propre bien-être, mais contribue également au «processus plus étendu de développement et de prospérité de la société (FIDA, 2019)».

Enfin, attirer et retenir les jeunes dans l'agriculture est essentiel pour réduire le chômage, les migrations de détresse et la pauvreté. Les jeunes sont trois fois plus susceptibles d'être au chômage que les adultes, les taux de chômage des jeunes stagnant obstinément à environ $14 \%$ dans le monde et atteignant jusqu'à $30 \%$ en Afrique du Nord (OIT, 2020). Les jeunes sont également plus susceptibles que les adultes d'occuper un emploi précaire et informel et de subir des conditions de travail abusives (Nations Unies, 2013). Le chômage et le sousemploi en plein essor alimentent à leur tour la migration rurale-urbaine et internationale, car de nombreux jeunes ruraux quittent leur foyer à la recherche d'un avenir meilleur. Des données récentes suggèrent que 32 pour cent des migrants internationaux ont moins de 30 ans (PNUD, 2015). Étant donné que le secteur urbain n'a que des capacités limitées pour absorber les jeunes ruraux qui migrent vers les villes dans de nombreux pays et régions en développement d'aujourd'hui, la promotion du travail indépendant dans les chaînes de valeur agricoles, tant dans les activités de production que dans celles d'après récolte, reste la stratégie de promotion de l'emploi la plus réaliste que les gouvernements ont à leur disposition.

\footnotetext{
2 Le CNS-FAO, I'OFAG, la FAO, l'Organisation mondiale des agriculteurs, le Brésil, YPARD et le GFRAS ont organisé conjointement l'événement parallèle "L'agriculture vous croyez que c'est pas cool?! Réfléchissez-y I Combler le fossé des générations » à la 45e session du Comité de la sécurité alimentaire mondiale en 2018. Les principaux résultats de la table ronde ont été publiés ici: http://www.fao.org/in-action/responsibleagricultural-investments/ news / detail / fr / c / 1161793 /
} 
Pourtant, comme le conclut une publication conjointe de la FAO, du CTA (Centre technique pour la coopération agricole et rurale) et du FIDA, «très peu de jeunes entrevoient un avenir dans l'agriculture» (FAO, CTA et FIDA, 2014). Cela n'a rien d'étonnant. Les jeunes qui veulent s'engager dans l'agriculture et l'agro-industrie font face à des obstacles importants qui les découragent ou les empêchent de lancer leur entreprise ou de la rendre économiquement viable. II s'agit notamment d'un accès limité aux connaissances, aux services financiers, à la terre et aux marchés (FAO, CTA et FIDA, 2014). Ces défis sont interdépendants, car des marchés de capitaux imparfaits empêchent l'acquisition de facteurs de production. Alors que les défaillances du marché peuvent dans de nombreux cas constituer des obstacles pour les jeunes agri-entrepreneurs, les gouvernements pourraient s'attaquer à ces problèmes et à d'autres problèmes liés aux politiques grâce à une stratégie cohérente, appliquée efficacement pour impliquer les jeunes dans l'agriculture et l'agro-industrie.

Reconnaissant la nécessité d'intensifier et d'autonomiser les jeunes dans l'agriculture, les décideurs ont pris une série d'engagements axés sur les jeunes aux niveaux mondial et régional. Le $4^{e}$ Principe pour un investissement responsable dans l'agriculture et les systèmes alimentaires, approuvé par le Comité des Nations Unies sur la sécurité alimentaire mondiale, appelle à «faire participer et autonomiser les jeunes» (CFS, 2014). La Déclaration de Malabo de l'Union africaine de 2014 sur la croissance et la transformation accélérées de l'agriculture pour une prospérité partagée et des moyens de subsistance améliorés engage les États membres à créer des opportunités d'emploi pour au moins 30 pour cent des jeunes dans les filières agricoles. Enfin, la sixième cible de l'Objectif 8 de développement durable («Promouvoir une croissance économique soutenue, inclusive et durable, le plein emploi productif et un travail décent pour tous») vise à «réduire considérablement la proportion de jeunes sans emploi, sans éducation ni formation» jusqu'en 2020.

\section{Aider les gouvernements à créer un environnement favorable aux jeunes agri-entrepreneurs.}

Pour traduire ces engagements mondiaux et régionaux en actions et en résultats concrets au niveau national, il faut comprendre clairement les besoins des jeunes femmes et hommes qui investissent dans les filières agricoles. Sur la base de cette compréhension commune, des cadres politiques, juridiques et incitatifs favorables doivent être conçus ou renforcés en collaboration avec les jeunes grâce à des processus d'élaboration de politiques participatifs.

Ce rapport vise à apporter une contribution utile à cet égard. II résume les principales conclusions, leçons apprises et bonnes pratiques d'une série d'analyses et de processus de planification multipartites inclusifs que la FAO a menés entre 2017 et 2020 dans onze pays africains - Côte d'Ivoire, Guinée, Malawi, Mali, Mauritanie, Mozambique, Namibie, Sénégal, Afrique du Sud, Tunisie et Ouganda — avec le généreux soutien de l'Office fédéral suisse de l'agriculture (OFAG). Ces analyses visaient à identifier les principales insuffisances en matière de politiques et de capacités et trouver des solutions pratiques pour autonomiser les jeunes et leur permettre d'investir dans l'agriculture et les systèmes alimentaires, tout en garantissant la viabilité économique et la durabilité de leurs investissements. 
La nécessité de renforcer les «incitations à l'investissement» spécifiques aux jeunes (voir encadré 1 pour une définition) est l'en des résultats majeurs de cette analyse. En fait, pour stimuler les investissements des jeunes agri-entrepreneurs et offrir aux jeunes une opportunité dans les filières agricoles, il faut des mesures d'incitation à l'investissement bien calibrées qui ciblent les jeunes ou des tiers (par exemple les institutions financières) qui investissent dans ces derniers. Ces incitations à l'investissement devraient être la pierre angulaire des stratégies gouvernementales visant à attirer les jeunes vers l'agriculture et l'agro-industrie et à leur donner les moyens d'investir. Ce rapport a pour objectif d'apporter une contribution utile à cet effort en partageant les résultats pratiques et les bonnes pratiques sur les incitations spécifiques aux jeunes. En particulier, il fait valoir que les incitations financières sont essentielles pour stimuler l'investissement des jeunes agri-entrepreneurs, mais qu'elles doivent être complétées par d'autres types d'incitations tangibles et intangibles.

\section{Encadré 1: En quoi consistent les incitations à l'investissement?}

Les incitations à l'investissement peuvent être définies comme «des mesures ciblées conçues pour influencer la taille, l'emplacement, l'impact, le comportement ou le secteur d'un investissement» (Tavares et al., 2017). Elles peuvent être proposées par les gouvernements nationaux, régionaux ou locaux (CNUCED, 2003). Les plans d'incitation à l'investissement peuvent englober un large éventail de mesures, notamment des incitations réglementaires, financières et fiscales, ainsi que des services techniques et des informations (Tavares-Lehmann, 2017). Jusqu'à récemment, les domaines de réflexion sur les incitations à l'investissement avaient dans de nombreux cas tendance à se limiter à des plans d'incitation (principalement fiscaux) pour l'investissement direct étranger (Tavares et al., 2017). Cependant, dernièrement, des efforts ont été faits pour «repenser les incitations à l'investissement» en envisageant d'y inclure différents types d'incitations à l'investissement et des considérations sur le développement durable.

Sans se limiter à créer et à mettre en œuvre des incitations axées sur les jeunes, il est tout aussi important de veiller à ce que la politique globale et les cadres juridiques autonomisent, sans défavoriser par inadvertance, les jeunes agri-entrepreneurs. La mise en œuvre efficace des politiques et des lois, ainsi que l'amélioration de l'efficacité et de la transparence des processus sont des défis importants à relever.

Enfin, il est essentiel d'impliquer les organisations de jeunes agri-entrepreneurs dans les processus d'élaboration des politiques et de donner la parole aux personnes réellement concernées. Cela nécessite à la fois de renforcer le dialogue inclusif grâce à des plates- 
formes multipartites institutionnalisées et de consolider de manière ciblée les capacités des organisations de jeunesse pour leur permettre de représenter les intérêts de leurs membres.

Le présent rapport développe ces principales conclusions. La section I présentera les méthodologies et les outils qui ont été appliqués. La section II examinera certains des indicateurs socio-économiques clés des onze pays africains et proposera un regroupement en différents groupes. La section III examinera certaines des principales conclusions et des recommandations politiques concernant l'accès des jeunes au financement, à la terre, aux services techniques et aux informations. Elle traitera de l'importance de la participation des jeunes aux processus d'élaboration des politiques. La section IV présentera cinq recommandations politiques clés relatives à l'autonomisation des jeunes agri-entrepreneurs. Enfin, une série de remarques finales et de suggestions d'activités de suivi sont en cours de formulation.

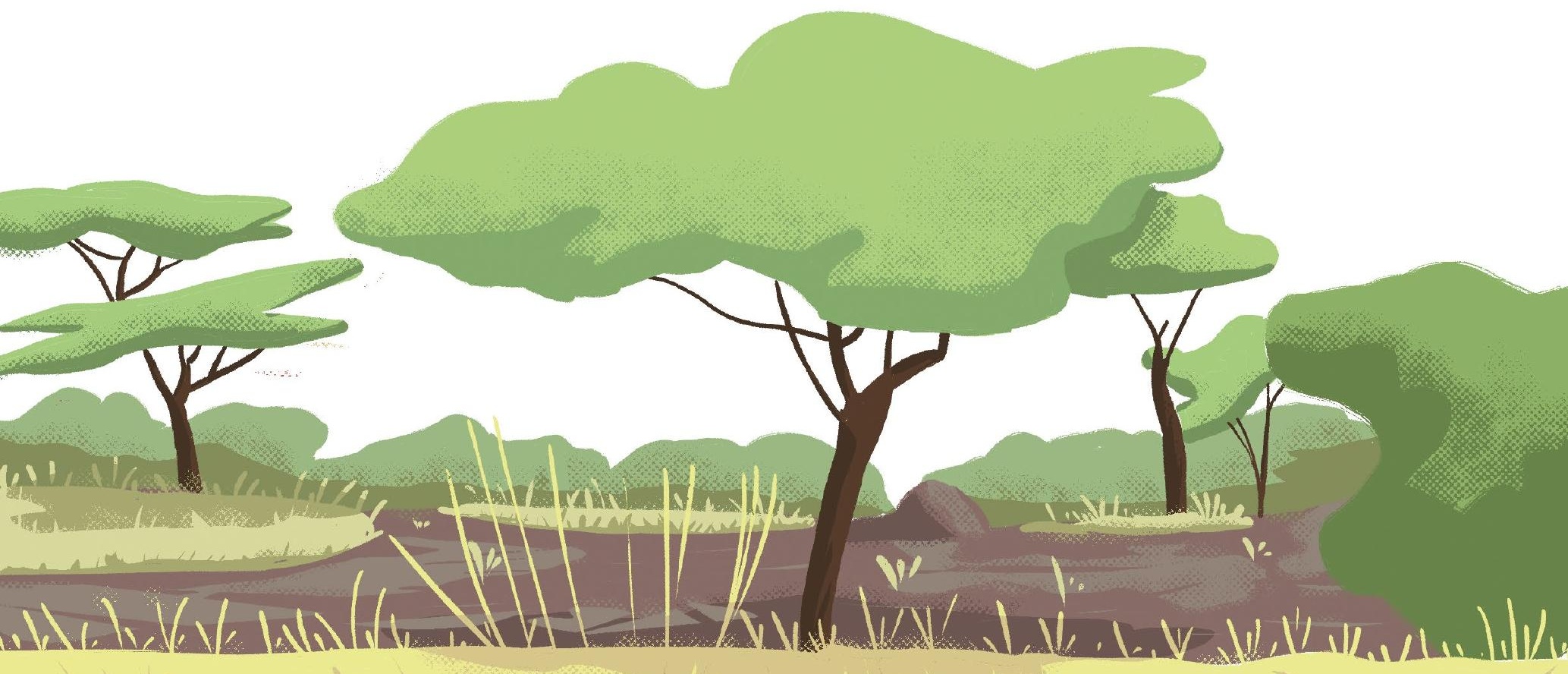




\section{MÉTHODOLOGIE}

Ce document résume les principales conclusions et leçons tirées de l'analyse et du travail de planification stratégique de la FAO avec onze pays africains sur la jeunesse et I'investissement responsable. Le projet de la FAO «Soutenir l'investissement responsable dans l'agriculture et les systèmes alimentaires» (GCP/GLO/886/MUL) visait à aider les acteurs étatiques et non étatiques à permettre aux jeunes à effectuer des investissements responsables dans l'agriculture et les systèmes alimentaires grâce à une analyse et une évaluation participatives des cadres politiques, juridiques et incitatifs actuels, des services existants fournis par des entités publiques et privées, et finalement du rôle des organisations de jeunesse dans l'autonomisation de leurs pairs. Sur la base de cette analyse, les parties prenantes nationales ont élaboré une série de recommandations dans les domaines d'intervention prioritaires. Le projet a été mis en œuvre en Afrique du Sud, en Côte d'Ivoire, en Guinée Conakry, au Malawi, au Mali, en Mauritanie, au Mozambique, en Namibie, au Sénégal, en Tunisie et en Ouganda.

Des analyses au niveau des pays ont été effectuées sur la base de la Boîte à outils de planification stratégique "Permettre aux jeunes agri-entrepreneurs d'investir dans l'agriculture et les systèmes alimentaires», qui comprend un outil d'analyse rapide des capacités (R-CAT) et un outil de planification stratégique.

Le R-CAT a été mis au point entre 2017 et 2018 et vise à aider les praticiens (tels que les agences gouvernementales, les organisations de jeunesse ou les partenaires de développement) à réaliser une analyse dans le cadre d'un atelier multi-acteurs de trois jours (FAO, 2018b, 2018 c). Le processus et l'outil s'inspirent des «questionnaires relatifs à l'évaluation des capacités ${ }^{3} »$ de la FAO et l'outil comprend quatre sections principales avec des questions spécifiques sur les sujets suivants:

1 Cadres institutionnels et participation des jeunes: cette section vise à (i) identifier les acteurs, organisations et entités actuellement impliqués dans les processus d'élaboration des politiques liées à l'investissement agricole; (ii) analyser l'inclusion et la participation des organisations de jeunesse dans ces processus; et (iii) identifier les plateformes multipartites qui servent ou pourraient servir de véhicules pour l'engagement des jeunes dans les processus d'élaboration des politiques;

2 Cadres politiques, juridiques et mesures d'incitation: cette section vise à évaluer si (i) les cadres politiques, juridiques, et les mesures d'incitation existants répondent suffisamment aux besoins des jeunes agri-entrepreneurs; (ii) des problèmes de mise en œuvre surviennent; (iii) des lacunes politiques existent.

3 Disponibilité et accessibilité des services: cette section vise à analyser si les services essentiels (services financiers, facilitation de l'accès à la terre, services de préparation) sont disponibles et accessibles, et comment les capacités des organisations de jeunesse pourraient être renforcées.

\footnotetext{
3 Voir le chapitre 2 du 2e module d'apprentissage de la FAO sur le développement des capacités pour plus d'informations sur les évaluations des capacités (FAO, 2012b).
} 
4 Programmes d'éducation formelle et informelle: cette section se concentre spécifiquement sur les programmes éducatifs pertinents pour les jeunes qui désirent commencer une carrière dans l'agriculture et les systèmes alimentaires. Elle évalue aussi leur disponibilité et leur accessibilité.

Avant l'atelier, une étude de base contenant un aperçu général spécifique au contexte de chacun de ces thèmes ainsi que des tendances socio-économiques pertinentes doit être rédigée pour orienter les discussions. Au cours de l'atelier, des représentants de différents groupes de parties prenantes (gouvernement, jeunes et leurs organisations, institutions financières et banques, ONG, universités et secteur privé) répondent collectivement aux différentes questions, guidés par un groupe de facilitateurs.

La FAO a employé le R-CAT dans une série d'ateliers d'évaluation des capacités multiacteurs avec dix pays africains pour soutenir une évaluation des obstacles que les jeunes doivent surmonter dans les systèmes agricoles et alimentaires et pour affiner l'outil en intégrant les leçons apprises. Deux ateliers nationaux multipartites d'analyse des capacités ont été organisés en Ouganda (novembre 2017) et en Côte d'Ivoire (avril 2018). Deux autres ateliers sous-régionaux ont été organisés avec quatre pays de la région de la SADC (Malawi, Mozambique, Namibie et Afrique du Sud) et des pays de la région du bassin du fleuve Sénégal (Guinée, Mali, Mauritanie et Sénégal) en mars et décembre 2018 respectivement. Des documents d'information ont été préparés par des consultants indépendants et des universitaires de diverses universités et institutions académiques. Un rapport intérimaire résumant les résultats des évaluations réalisées entre novembre 2017 et avril 2018 a été publié par la FAO (FAO, 2019).

L'outil de planification stratégique (Eiselen et Fiedler, 2020) a été mis au point entre 2019 et 2020 à la suite d'un examen du R-CAT par le Comité national suisse de la FAO. L'outil de planification stratégique intègre des éléments importants du R-CAT, mais suit une approche plus complexe qui conduit à des recommandations plus ciblées, élaborées et spécifiques. L'outil de planification stratégique associe des recherches et des entretiens d'experts à des consultations sur le terrain et des ateliers multipartites. Ses cinq principales étapes consécutives sont les suivantes:

1 Analyse de la situation et du contexte: une étude qui analyse (i) les principales tendances socio-économiques (investissements, emploi par âge, éducation et sexe, principales chaînes de valeur) ventilées par régions infranationales permettant d'identifier les domaines d'intervention prioritaires (régions, filières clés, catégories spécifiques de jeunes les plus susceptibles de devenir des agri-entrepreneurs compétitifs et ayant besoin de soutien; (ii) et les cadres institutionnels, politiques, juridiques, les mesures d'incitations actuels et les services existants (à l'aide du questionnaire R-CAT);

2 Identification et qualification des parties prenantes: les entretiens d'experts et les consultations sur le terrain aboutissent à l'identification des parties prenantes clés engagées dans des filières sélectionnées et dans les processus d'élaboration des politiques; une évaluation de la contribution de ces parties prenantes à l'autonomisation 
des jeunes dans les systèmes agricoles et agro-alimentaires sur la base d'une liste de critères et de repères spécifiques; et une cartographie des parties prenantes.

3 Création d'une vision commune: tous les acteurs concernés travaillent ensemble dans un atelier multipartite pour identifier une vision commune des changements clés nécessaires qui permettront aux jeunes agri-entrepreneurs d'investir, sur la base des résultats des deux premières étapes.

4 Proposer des solutions concrètes: la vision commune est confrontée à l'analyse de la situation et des solutions concrètes sont recherchées.

5 Validation: les solutions proposées sont validées lors d'un atelier multipartite.

L'outil de planification stratégique a été appliqué par le Laboratoire d'économie rurale de l'Institut national de la recherche agronomique de Tunisie (INRAT) entre juin 2019 et mars 2020. Le projet n'a pas simplement formulé des recommandations concrètes et opérationnelles, non négligeables, mais il a aussi favorisé la collaboration et le dialogue entre le gouvernement et le monde universitaire.

Les résultats produits par le R-CAT et l'outil de planification stratégique ont été complétés par des recherches supplémentaires. En particulier, ce document a bénéficié des renseignements de deux études supplémentaires ayant adopté une méthodologie similaire. Celles-ci, commandées pour un programme d'apprentissage connexe, comprenaient des analyses sur les difficultés, les opportunités et les bonnes pratiques en Namibie et en Afrique du Sud. Par ailleurs, lorsque cela était nécessaire et pertinent, l'auteur a effectué des recherches documentaires supplémentaires pour vérifier ou compléter les informations et les données.

Par conséquent, les résultats présentés dans ce document sont le fruit d'un processus collectif impliquant un large éventail de parties prenantes et d'experts.

Pour toutes les études et évaluations entreprises, le terme «jeunes» regroupe l'ensemble des femmes et des hommes âgés de 15 à 35 ans, conformément à la définition officielle adoptée par l'Union africaine (2006). Étant donné que les travaux entrepris sont spécifiques au contexte africain, cette définition a été retenue à la place de celle des Nations Unies (1524 ans, Nations Unies, 2019) qui est plus restrictive. 



\section{UN APERÇU SOCIO-ÉCONOMIQUE DE L'ÉTAT DE LA JEUNESSE ET DE L'AGRICULTURE}

Les onze pays africains dans lesquels la FAO a réalisé les différentes études peuvent être regroupés en trois groupes: un groupe constitué d'économies rurales à faible revenu dépendant de l'agriculture (Guinée, Mali, Malawi, Mozambique et Ouganda); un deuxième groupe composé d'économies à revenu intermédiaire de la tranche inférieure dont les zones rurales enregistrent une certaine transformation (Côte d'Ivoire, Mauritanie et Sénégal); et un troisième groupe de pays aux économies très diversifiées et dont la transformation des zones rurales est avancée (Namibie, Afrique du Sud et Tunisie).

\section{Agriculture et productivité agricole}

Dans le groupe 1 (voir Figure 1), 65 pour cent (Mali) à 72 pour cent (Malawi, Mozambique) de la population totale sont employés dans l'agriculture. La part de la valeur ajoutée brute (VAB) de l'agriculture dans le produit intérieur brut (PIB) est beaucoup plus faible, environ un tiers dans la plupart des pays (sauf au Mali, où elle représente environ la moitié du rapport agriculture-emploi total), avec des taux allant de 16 pour cent (Guinée) à 38 pour cent (Mali). Cela s'explique par une très faible valeur ajoutée agricole par travailleur (environ 500 USD au Mozambique et en Ouganda; au Mali, ce montant qui atteint 1623 USD, est trois fois supérieur). Dans ces pays, il existe un énorme potentiel pour les investissements qui améliorent la productivité et la valeur ajoutée globale du secteur agricole.

Dans le groupe 2 (voir Figure 2), les économies sont moins dépendantes de l'agriculture. Le secteur agricole représente 15 pour cent (Sénégal) à 23 pour cent (Côte d'lvoire) du PIB et emploie également une part beaucoup plus faible de la population - entre 32 pour cent (Sénégal) et environ 50 pour cent (Côte d'Ivoire et Mauritanie). La valeur ajoutée par travailleur dans le secteur agricole est également considérablement plus élevée que celle du groupe 1, avec des taux compris entre environ 2000 USD (Côte d'lvoire), 2400 USD (Sénégal) et 4500 USD (Mauritanie). Dans ces pays, certaines filières sont très développées et intégrées aux marchés internationaux (comme le cacao en Côte d'Ivoire), tandis que d'autres ont un potentiel élevé qui pourrait être davantage exploité grâce à des investissements importants (cultures de contre-saison dans la région du bassin du fleuve Sénégal, bétail/viande rouge en Mauritanie).

Le groupe 3 (voir Figure 3) est caractérisé par des économies diversifiées, dans lesquelles l'agriculture ne représente qu'une faible part de l'emploi total (entre 5 pour cent en Afrique du Sud et 20 pour cent en Namibie) et du PIB (entre 2 pour cent en Afrique du Sud et 10 pour cent en Tunisie). Les filières agricoles sont beaucoup plus développées et le secteur agricole est plus productif, comme en témoigne la plus grande valeur ajoutée par travailleur agricole (entre environ 7000 USD en Namibie et 12000 USD en Afrique 
du Sud). Dans ces pays, les investissements pourraient avoir un impact transformateur en augmentant la valeur ajoutée le long des filières agricoles, notamment par le biais de labels tels que des indicateurs géographiques, et en se concentrant sur les marchés émergents et de niche (agrotourisme et micro-filières haut de gamme pour les marchés locaux).

Figure 1: Valeur ajoutée brute de l'agriculture (\% du PIB), emploi dans l'agriculture (\% de l'emploi total) et valeur ajoutée agricole par travailleur (en USD) en Guinée, au Malawi, au Mali, au Mozambique et en Ouganda

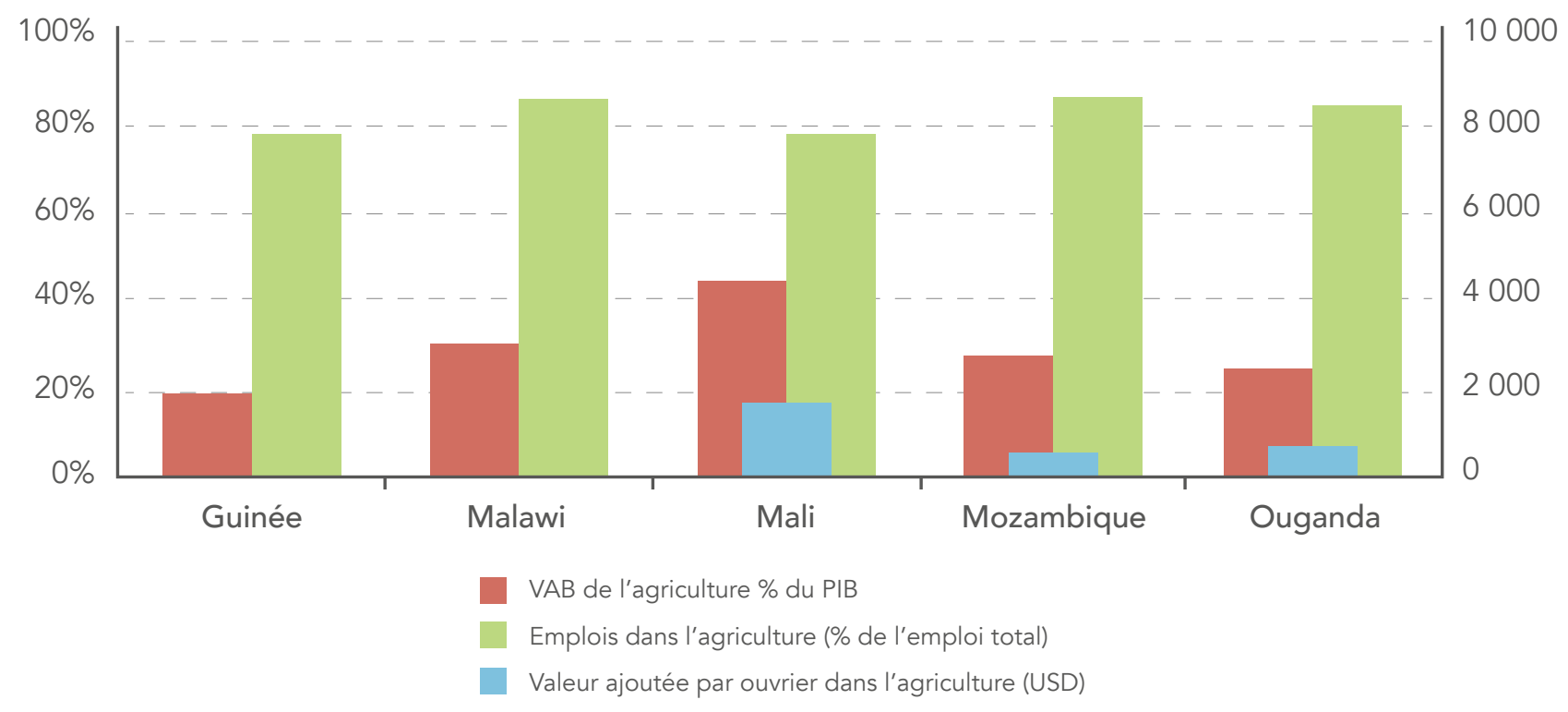

Source: Figure élaboré par l'auteur à partir de FAOSTAT (VAB agriculture, données 2018; valeur ajoutée agricole par travailleur, données les plus récentes disponibles) et indicateurs du développement mondial (emploi dans l'agriculture, données 2018). Données récupérées en juin 2020.

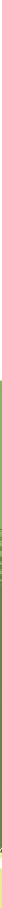


Figure 2: Valeur ajoutée brute de l'agriculture (\% du PIB), emploi dans l'agriculture (\% de l'emploi total) et valeur ajoutée agricole par travailleur (en USD) en Côte d'Ivoire, en Mauritanie et au Sénégal

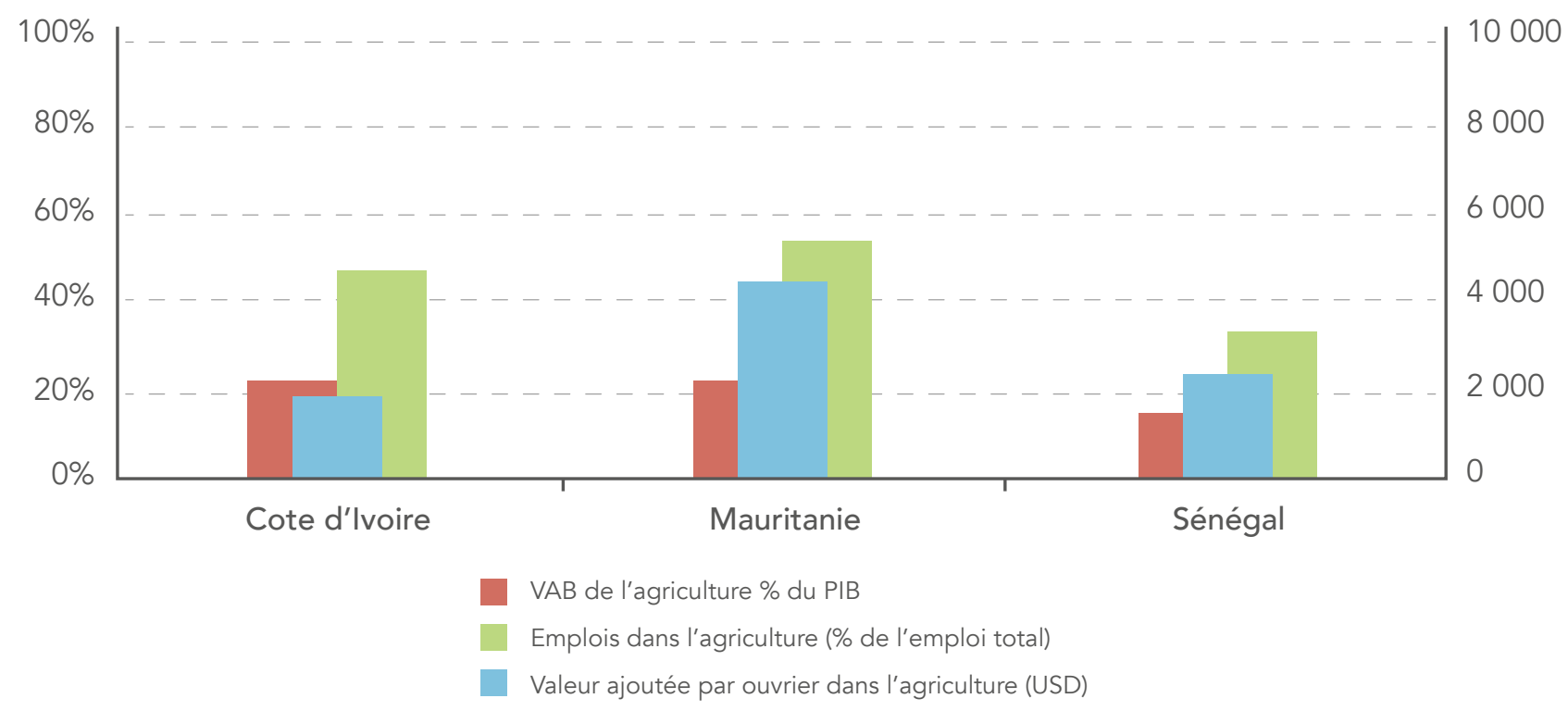

Source: Figure élaboré par l'auteur en se basant sur FAOSTAT (VAB agriculture, données 2018; valeur ajoutée agricole par travailleur, données les plus récentes disponibles) et indicateurs du développement mondial (emploi dans l'agriculture, données 2018). Données récupérées en juin 2020.

Figure 3: Valeur ajoutée brute de l'agriculture (\% du PIB), emploi dans l'agriculture (\% de l'emploi total) et valeur ajoutée agricole par travailleur (en USD) en Namibie, Afrique du Sud et Tunisie

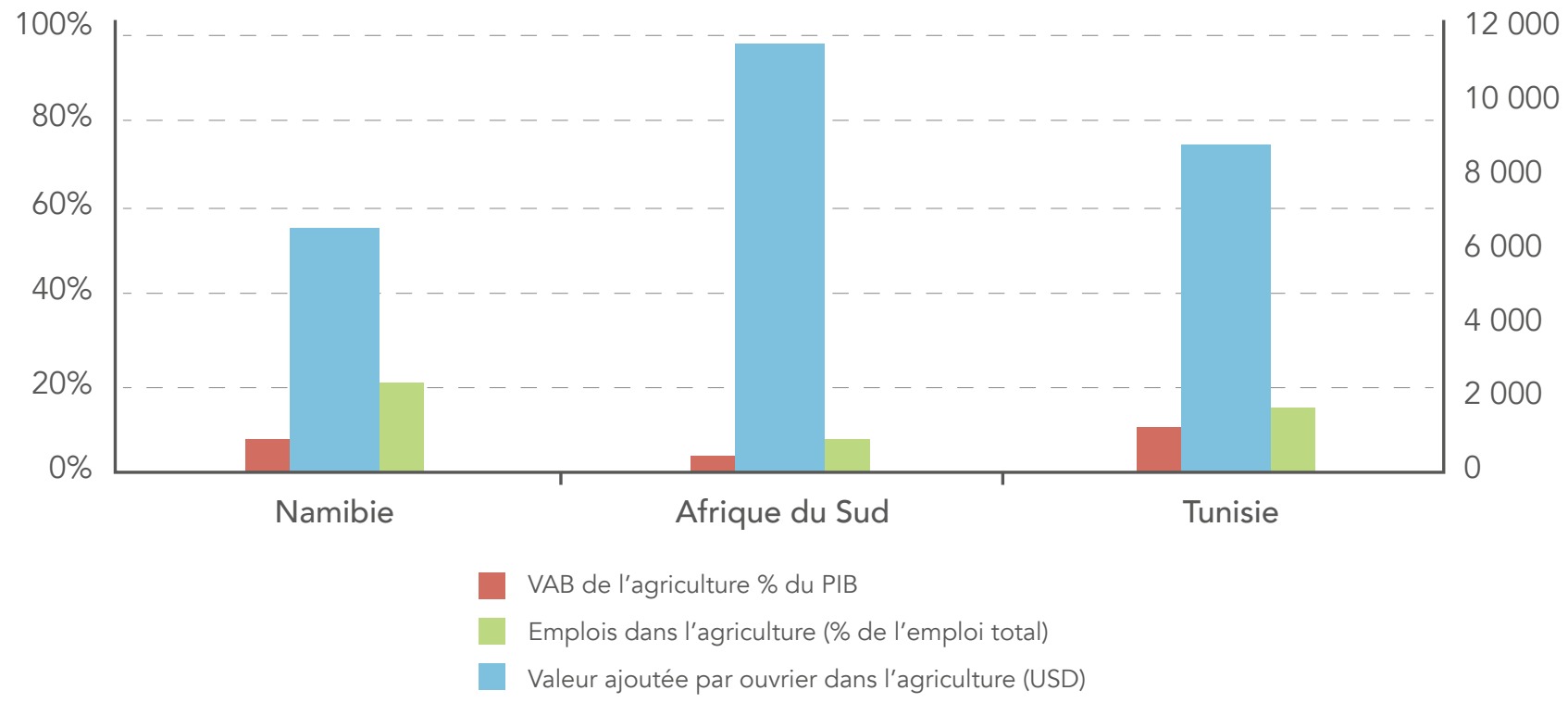

Source: Figure élaboré par l'auteur à partir de FAOSTAT (VAB agriculture, données 2018; valeur ajoutée agricole par travailleur, données les plus récentes disponibles) et des indicateurs du développement mondial (emploi dans l'agriculture, données 2018). Données récupérées en juin 2020. 


\section{Éducation des jeunes et chômage des jeunes}

L'analyse des taux de scolarisation dans l'enseignement secondaire et supérieur (voir Figure 4) confirme le regroupement en trois groupes proposé précédemment. Les taux de scolarisation sont les plus bas pour le Malawi, le Mozambique et l'Ouganda (moins de 10 pour cent) suivis de la Côte d'Ivoire, du Mali et de la Mauritanie (près de 15 pour cent pour l'enseignement secondaire, moins de 10 pour cent pour l'enseignement supérieur), la Guinée et le Sénégal enregistrant un taux de scolarisation légèrement plus élevé (17 et 19 pour cent pour l'enseignement secondaire, et 12 et 13 pour cent pour l'enseignement supérieur respectivement). Les taux de scolarisation en Namibie, en Afrique du Sud et en Tunisie sont considérablement plus élevés, atteignant respectivement 81 et 32 pour cent pour l'enseignement secondaire et supérieur en Tunisie.

Pourtant, en dépit de toutes les différences macroéconomiques et des normes d'éducation dans les différents pays, les perspectives pour les jeunes sont limitées partout. Le fait qu'il existe un énorme potentiel inexploité en termes de développement agricole dans le groupe 1 (et dans le groupe 2) n'a pas non plus abouti à la création de suffisamment d'opportunités d'emploi pour les jeunes. En même temps, le niveau d'éducation élevé du groupe 3 n'a pas non plus offert de meilleures perspectives aux jeunes. (voir Figure 5.) En fait, les taux de chômage des jeunes relativement faibles dans les groupes 1 et 2 (inférieurs à 10 pour cent dans la plupart des cas) doivent être lus conjointement avec des taux de sous-emploi des jeunes plus élevés et un taux global étonnamment élevé de personnes occupant un emploi vulnérable ${ }^{4}$ qui dépasse 50 pour cent dans tous les pays des groupes 1 et 2 et atteint même des taux supérieurs à 80 pour cent dans certains cas (Guinée, Mali et Mozambique). À l'inverse, les taux d'emploi vulnérable sont considérablement plus faibles en Namibie, en Afrique du Sud et en Tunisie (31, 10 et 20 pour cent respectivement), mais les taux de chômage des jeunes sont beaucoup plus élevés (38, 53 et 33 pour cent respectivement). En Tunisie, il existe une corrélation négative entre l'éducation et l'emploi, le taux de chômage des personnes sans diplôme s'établissant à 4,9 pour cent alors qu'il atteint 28,3 pour cent chez les diplômés universitaires (FAO et INRAT, 2020a). Par conséquent, les jeunes titulaires d'un diplôme de l'enseignement supérieur sont particulièrement susceptibles d'être au chômage. Dans le même temps, en Afrique du Sud, le chômage des jeunes diplômés de l'enseignement supérieur a augmenté davantage en 2018 que le chômage de toute autre catégorie de jeunes.

Ces tendances montrent que la disponibilité de ressources (humaines et naturelles) abondantes n'entraînera pas en soi une augmentation des investissements qui générera des opportunités d'emploi, et que, à l'inverse, «augmenter le niveau d'éducation de la main- $d$ 'œuvre émergente dans les pays en développement ne garantit pas en soi une intégration facile de la main-d'œuvre plus qualifiée dans des emplois non vulnérables» (OIT, 2014). Bien au contraire, des interventions politiques ciblées sont nécessaires pour stimuler et orienter les investissements qui génèrent des opportunités pour les jeunes, renforcent leurs capacités là où c'est nécessaire et tirent parti de celles qui existent déjà, et ont un impact transformateur en termes de développement territorial.

4 L'OIT définit l'emploi vulnérable comme la «proportion de travailleurs indépendants et de travailleurs familiaux contribuant à l'emploi total» (OIT, 2013). 
Figure 4: Éducation - taux de scolarisation par pays

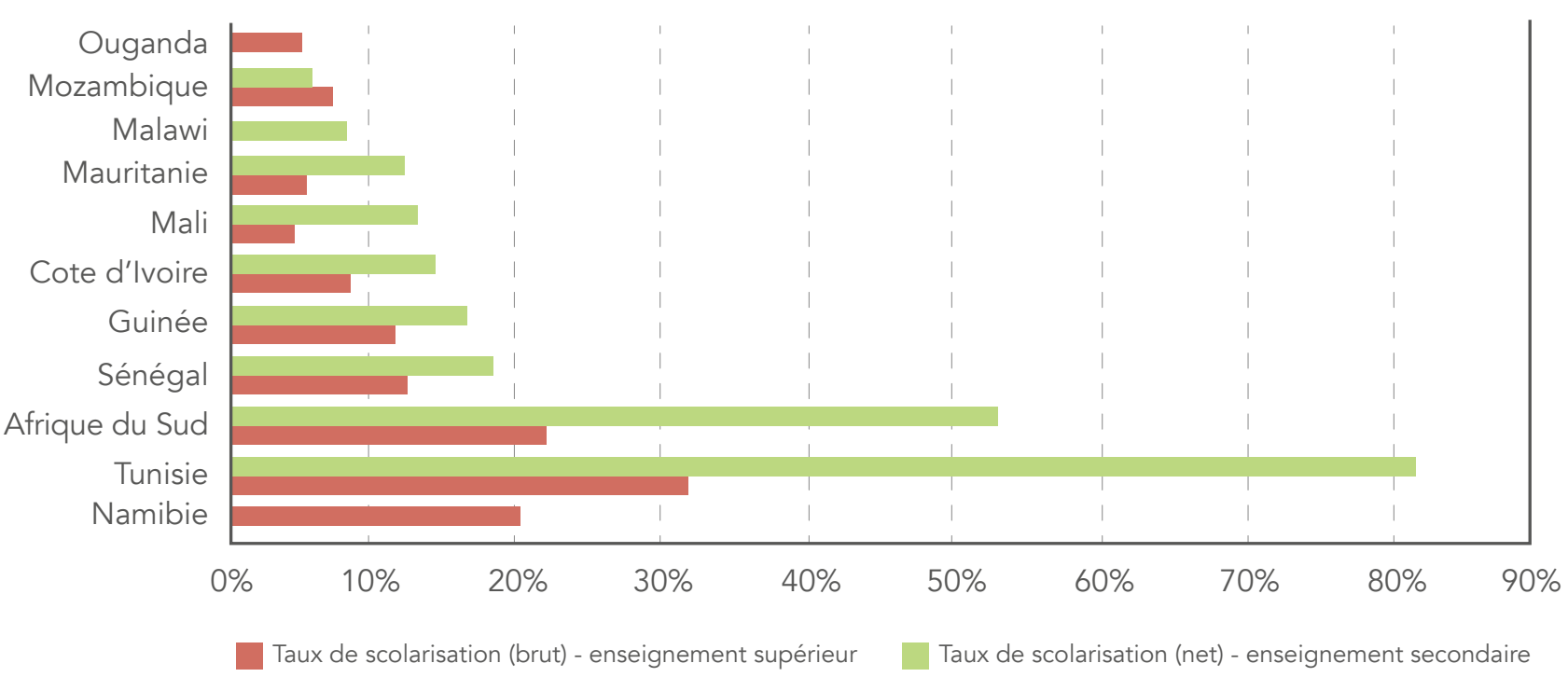

Source: Figure élaboré par l'auteur à partir des données de I'UNESCO. Données 2018 ou données les plus récentes disponibles. N. B.: aucune donnée disponible pour l'enseignement secondaire en Ouganda et en Namibie. Aucune donnée disponible pour les taux de scolarisation dans l'enseignement supérieur au Malawi.

Figure 5: Emploi vulnérable, sous-emploi et chômage des jeunes

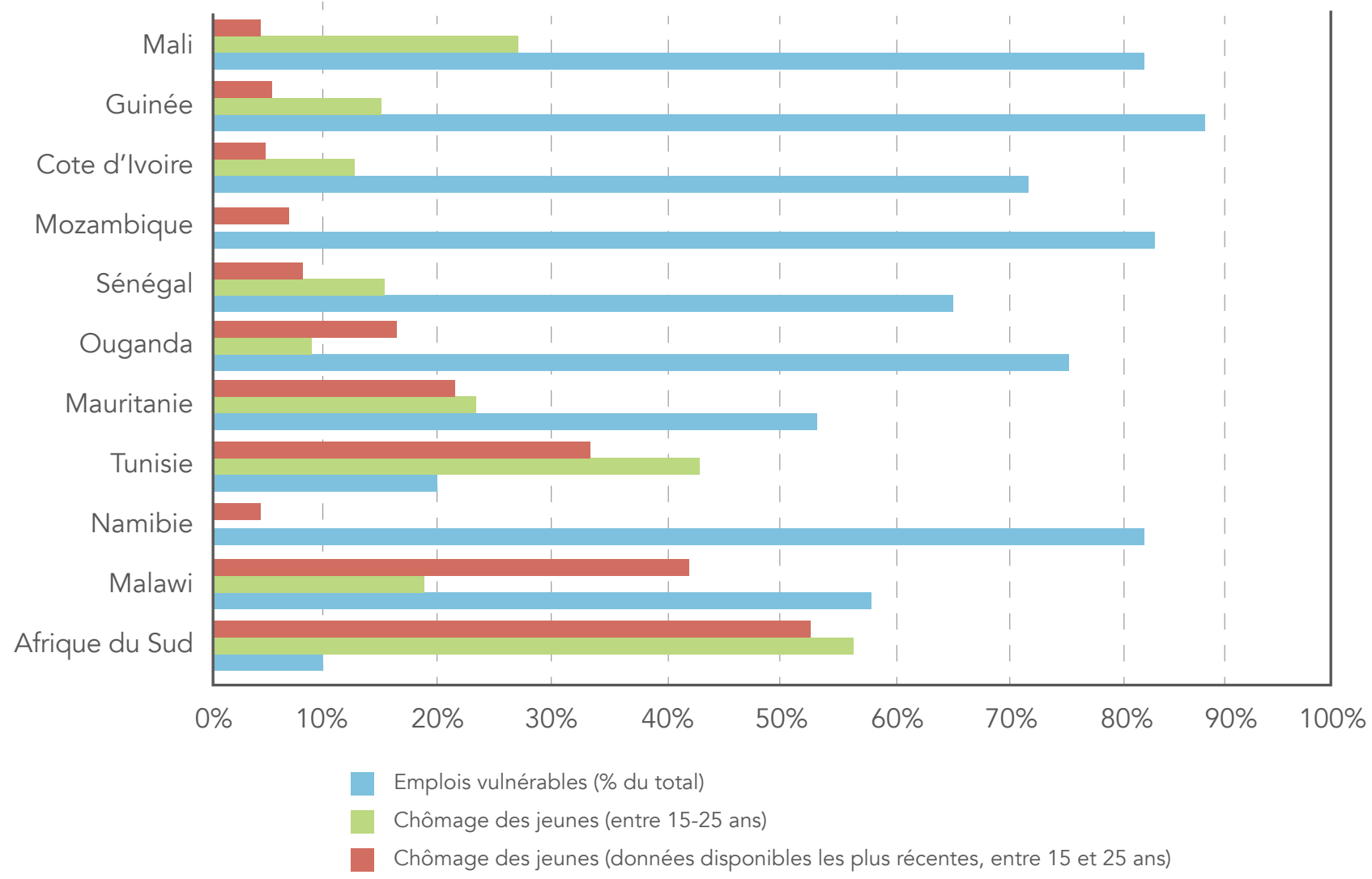

Source: Figure élaboré par l'auteur à partir des données d'ILOSTAT (2018 ou données les plus récentes antérieures à 2018); document d'information INRAT pour la Tunisie; indicateurs du développement mondial. 
En conclusion, dans les pays du groupe 1 et, dans une moindre mesure, dans les pays du groupe 2, l'agriculture représente toujours le principal secteur qui peut absorber un nombre élevé de jeunes entrant sur le marché du travail chaque année, selon les indicateurs socio-économiques actuels. Dans les pays du groupe 3, les investissements dans le secteur agricole, malgré l' importance décroissante de celui-ci, pourraient avoir un impact transformateur, notamment lorsqu'ils sont réalisés en aval de la chaîne de valeur et lorsqu'ils exploitent des potentiels actuellement sous-évalués tels que la labellisation (comme la certification de la performance sociale et environnementale ou des indications géographiques ${ }^{5}$ ) ou l'agritourisme, dans un contexte de taux de chômage particulièrement élevés en milieu rural.

\footnotetext{
${ }^{5}$ Selon la FAO (2018d), «les indications géographiques (IG) désignent des produits ayant des caractéristiques, qualités ou réputations spécifiques résultant de leur origine géographique. Cela différencie les produits en fonction de caractéristiques locales uniques, d'histoire ou de caractéristiques distinctives liées à des facteurs naturels et humains, tels que le sol, le climat, le savoir-faire local et les traditions. Les IG sont reconnues comme des droits de propriété intellectuelle (DPI) et offrent donc à la fois un outil de marketing utile et une protection du nom. »
}

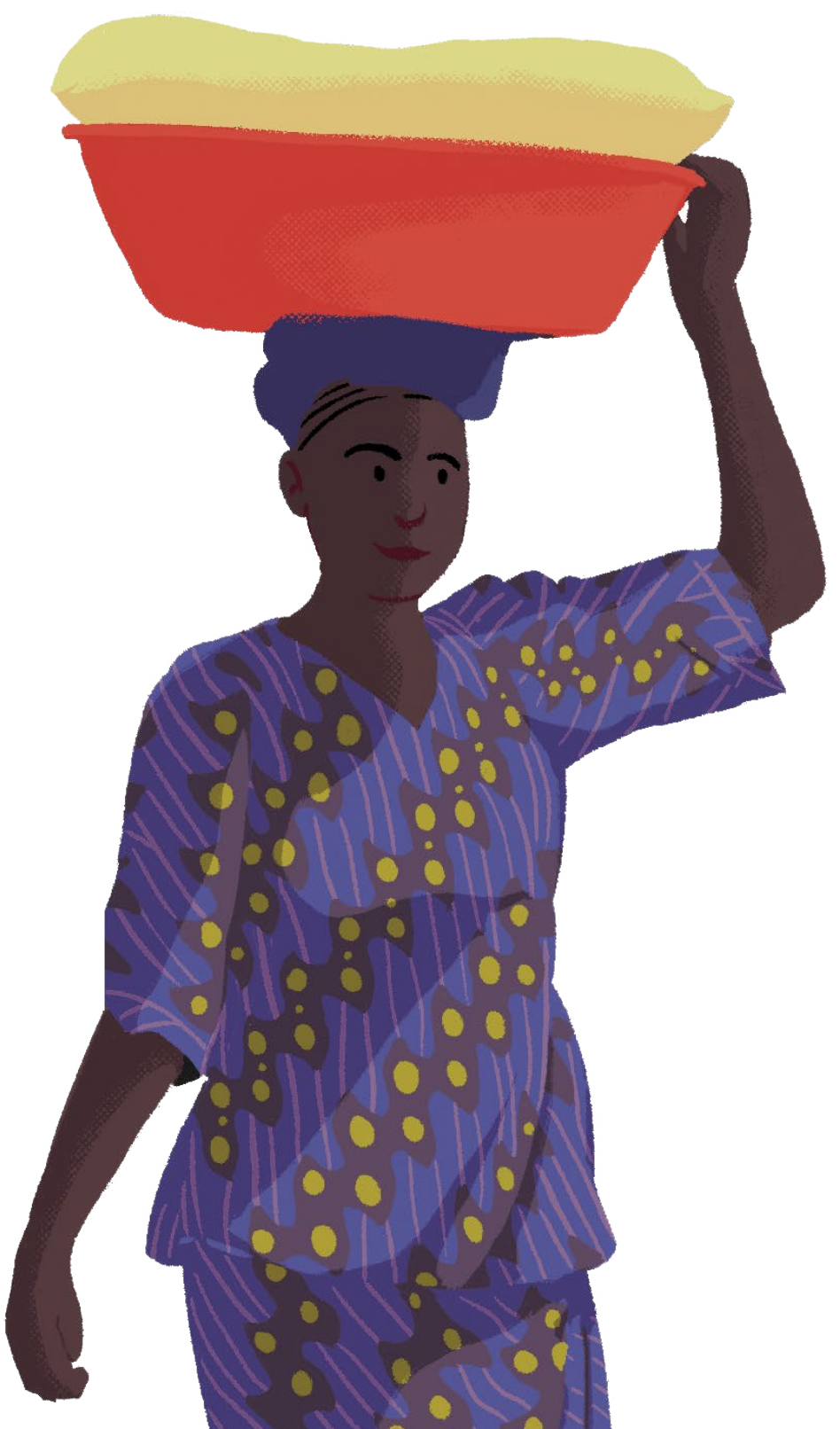




\section{PERMETTRE AUX JEUNES D'ACCÉDER AUX FACTEURS DE PRODUCTION ET AUX SERVICES D'APPUI TECHNIQUE}

Ce chapitre présente les principales conclusions des analyses des capacités menées par la $\mathrm{FAO}$, des processus de planification stratégique et des bonnes pratiques qui permettent aux jeunes d'accéder aux facteurs de production (capital et terre) et aux services d'appui technique essentiels pour assurer la viabilité économique de leurs investissements. Il examine le rôle spécifique que les incitations à l'investissement peuvent jouer pour surmonter les défaillances et les imperfections communes du marché et formule des recommandations politiques fondées sur ces analyses.

\section{Accès aux services financiers}

L'accès aux services financiers est un déterminant clé de la capacité des jeunes femmes et hommes à transformer leurs idées commerciales en réalité grâce à des investissements productifs. Les services financiers importants comprennent les prêts et les assurances ainsi que les comptes bancaires et les plans d'épargne. Pourtant, l'accès aux services financiers est un défi majeur pour les jeunes agri-entrepreneurs. La section suivante (1.1) traitera de l'accès des jeunes aux (i) services financiers commerciaux, incluant les comptes bancaires, les prêts et les assurances; et (ii) aux plans d'épargne informels et aux microcrédits. La section successive (1.2) présentera les programmes d'incitation gouvernementaux existants qui visent à remédier aux imperfections du marché. Enfin, la dernière section (1.3) fournira des recommandations politiques pour permettre aux jeunes d'accéder aux services financiers.

\subsection{Accès aux services financiers commerciaux et informels}

\section{(i) Services financiers commerciaux}

Les comptes bancaires permettent aux jeunes d'épargner un capital qui pourra être investi ultérieurement et de prouver leur aptitude à bénéficier de prêts bancaires commerciaux. Cependant, la prévalence des jeunes qui déclarent avoir des comptes bancaires dans des institutions financières est inférieure à 20 pour cent en Côte d'Ivoire, en Guinée, au Mali, en Mauritanie et au Sénégal, entre 20 et 50 pour cent au Malawi, au Mozambique, en Tunisie et en Ouganda et dépasse 50 pour cent seulement en Namibie et en Afrique du Sud (voir Figure 6). L'introduction de l'argent mobile a eu un impact significatif sur l'augmentation de la prévalence des comptes dans le premier groupe (en la portant à environ 30 pour cent sauf en Guinée, où le taux est légèrement supérieur à 20 pour cent), mais n'a eu pratiquement aucun impact dans le deuxième et le troisième groupe.

Dans ce contexte, l'accès à d'autres services financiers (systèmes de crédit et d'assurance) semble encore plus limité. Même si une analyse quantitative ne peut être entreprise en raison du manque de données fiables, les études, les groupes de discussion et les 
entretiens d'experts issus des analyses de capacités indiquent de sérieux problèmes à cet égard. Dans les pays où un système de notation a été utilisé pour évaluer l'accès des jeunes agri-entrepreneurs et agriculteurs à ces services financiers, en utilisant une fourchette comprise entre 0 (pas d'accès) et 5 (excellent accès), l'accès au crédit a obtenu une note de 2 en moyenne et l'accès à des systèmes d'assurance 1 (voir Figure 7). Les scores d'accès au crédit oscillaient régulièrement entre 1 (Guinée) et 2,5 (Sénégal). Dans les pays où aucun système de notation n'était utilisé, le manque d'accès à l'assurance agricole a également été mis en évidence (Malawi et Mozambique).

Figure 6: Jeunes qui déclarent avoir des comptes bancaires (\% du total des jeunes)

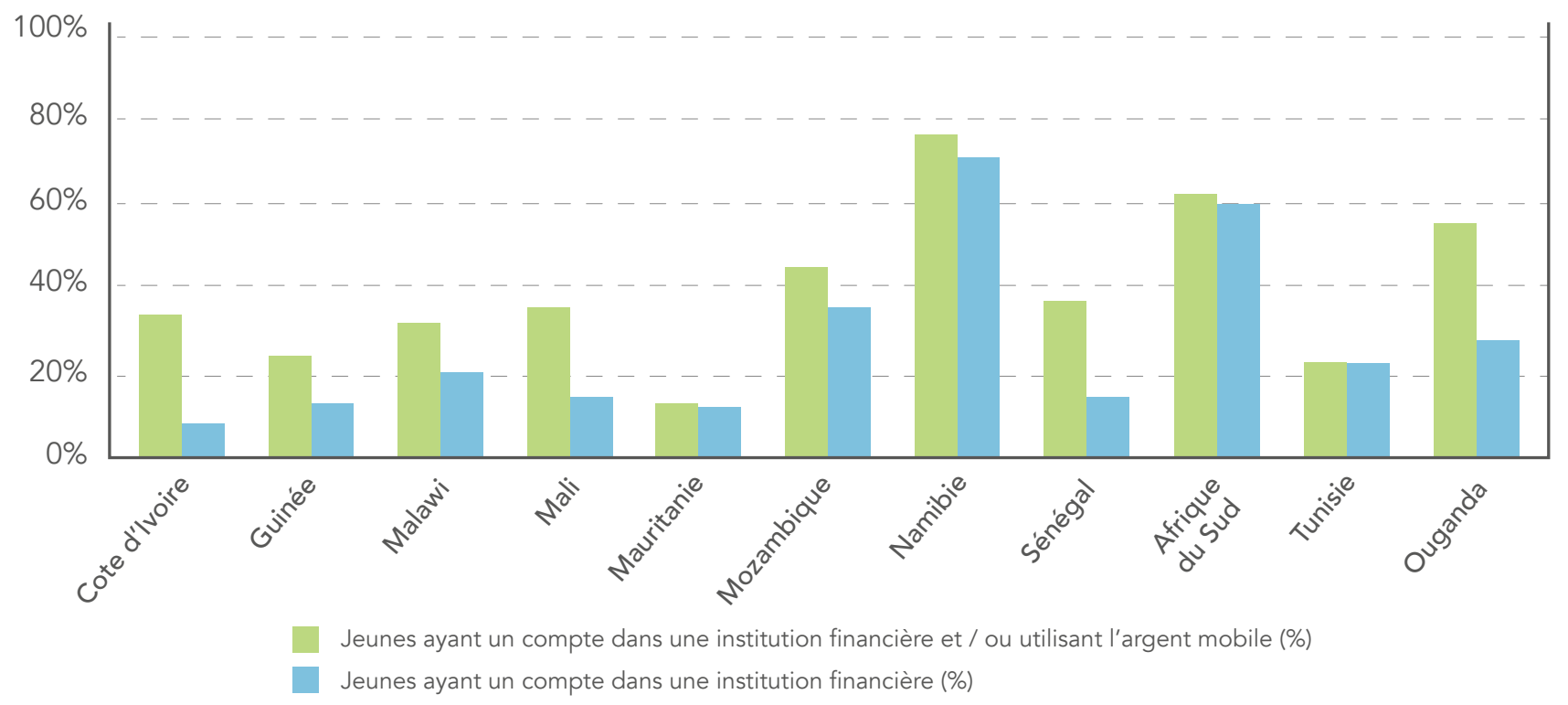

Source: Figure élaboré par l'auteur à partir de la base de données Global Findex. Données les plus récentes disponibles (2017).

Figure 7: Accès des jeunes agri-entrepreneurs et des jeunes agriculteurs aux services financiers, comme indiqué dans les évaluations des capacités $(0=$ pas d'accès, $5=$ excellent accès)

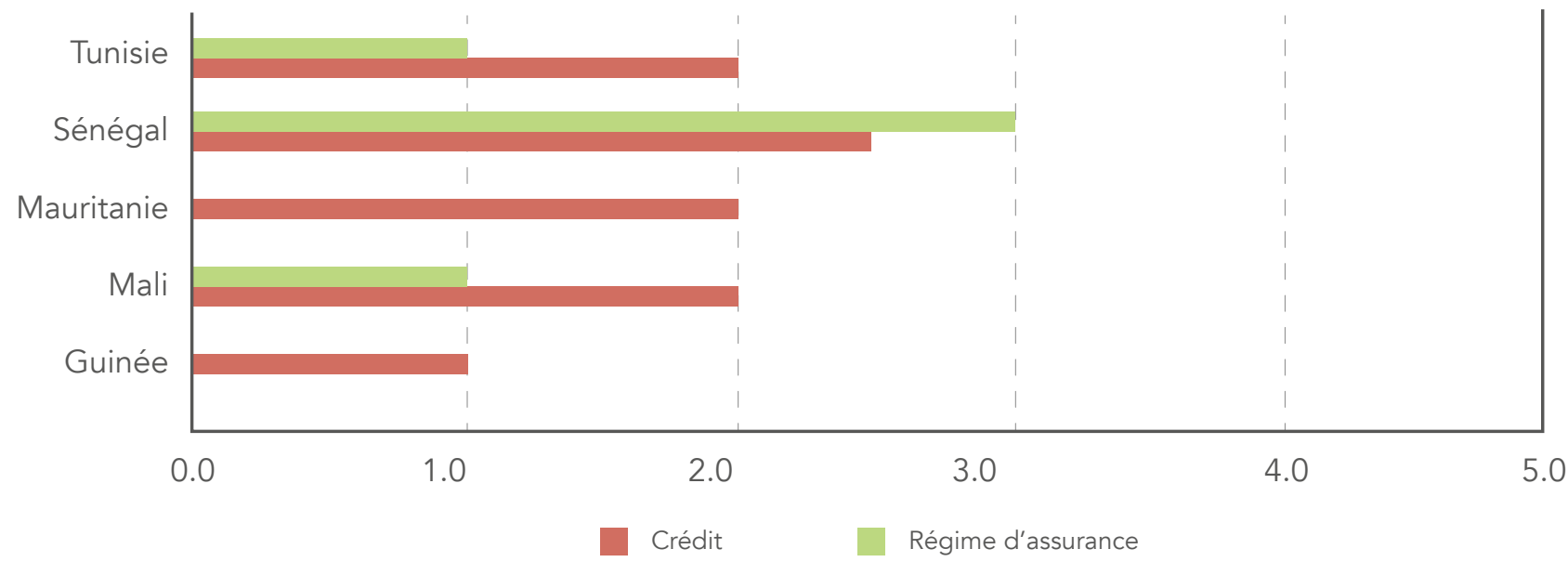

Source: Figure élaboré par l'auteur à partir de la base de données FAO RAl et des évaluations des capacités des jeunes, 2020. 
Figure 8: Crédit à l'agriculture (\% du total des crédits) et valeur ajoutée brute de l'agriculture (\% du PIB)

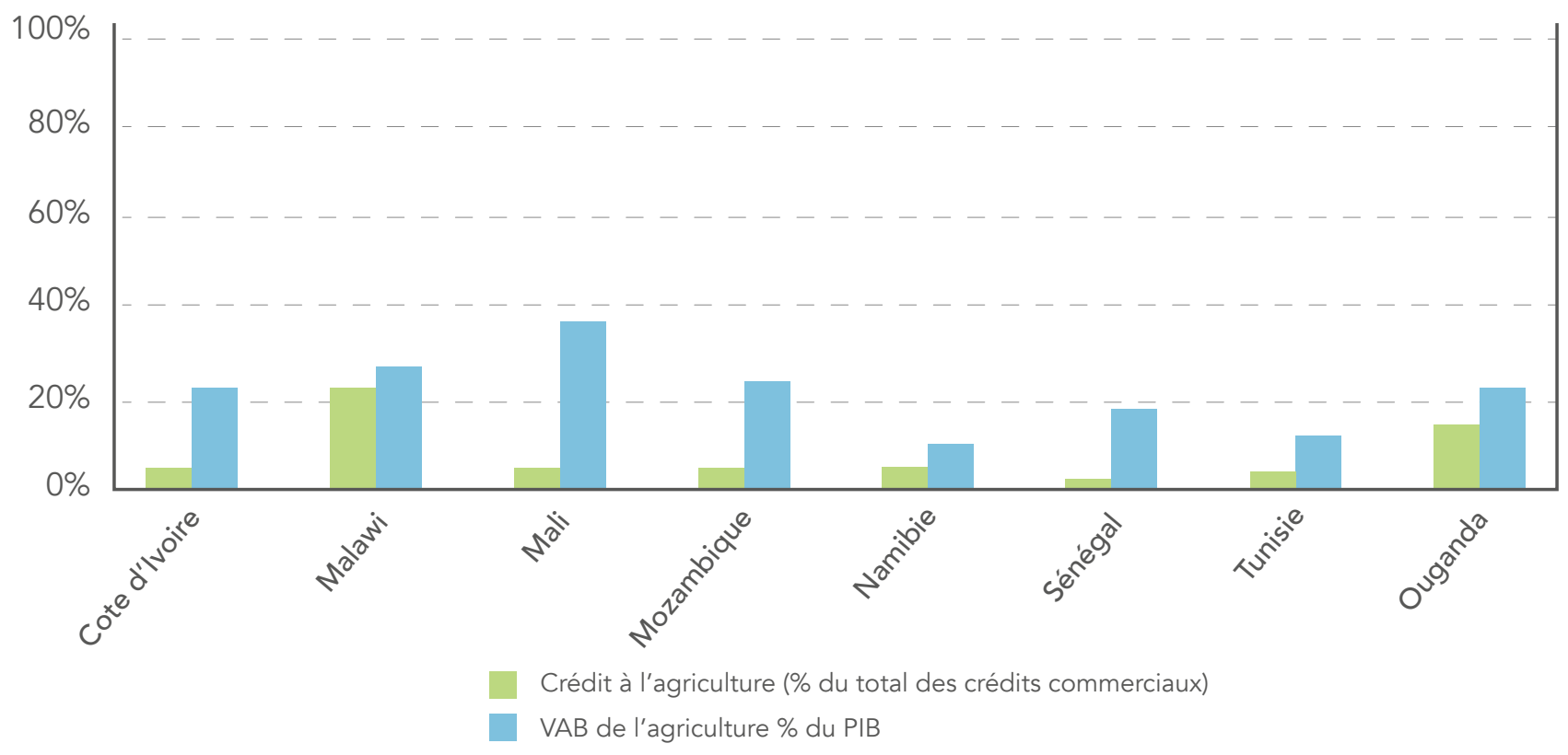

Source: Figure élaboré par l'auteur à partir des données FAOSTAT récupérées en avril 2020. VAB agriculture: données 2018. Crédit à l'agriculture: données les plus récentes disponibles.

Des marchés de capitaux imparfaits expliquent en partie le faible accès des jeunes agri-entrepreneurs aux services financiers commerciaux, notamment aux crédits et aux régimes d'assurance. Trois contraintes majeures peuvent être identifiées - (i) les difficultés spécifiques aux jeunes, (ii) les difficultés plus générales liées au secteur agricole, et (iii) la rareté des capitaux. Premièrement, les banques exigent généralement des garanties qui déterminent si un prêt est accordé et à quelles conditions. Les jeunes agri-entrepreneurs ne disposent généralement ni de flux de trésorerie importants ni d'économies substantielles pour atteindre un degré suffisant de capacité d'autofinancement, sans parler de garanties précieuses telles que des biens immobiliers, des capitaux propres ou d'autres actifs qu'une institution financière peut accepter comme garantie. Leur manque d'expérience perçu ou réel peut être un obstacle supplémentaire. Deuxièmement, la libéralisation du secteur financier n'a pas eu d'effets d'entraînement significatifs sur le crédit rural (Turvey, 2017) et il «semble que les producteurs agricoles soient confrontés à un préjugé négatif en ce qui concerne l'accès au crédit» (FAO, 2018a). Cela peut s'expliquer en partie par le risque plus élevé que représentent les investissements agricoles par rapport à d'autres investissements (par exemple, les obligations d'État ou les secteurs considérés comme moins risqués), ce qui peut constituer un défi particulier dans les pays où le secteur financier est sous-développé et le capital rare (Amadhila, non publié). Ceci est illustré par l'écart important entre la valeur ajoutée brute de l'agriculture au PIB et la part du crédit à l'agriculture dans le total des crédits, en particulier dans les pays du groupe 1, à l'exception du Malawi et de l'Ouganda (voir Figure 8).

À un autre niveau, il existe aussi des contraintes réglementaires. II s'agit ici de l'absence de réglementation des taux d'intérêt ou de taux de référence fixes généraux qui sont trop 
élevés pour les jeunes agri-entrepreneurs qui doivent déjà payer des frais plus élevés en raison de leur profil de risque plus élevé. Par conséquent, en Tunisie, I'un des principaux résultats du processus de planification multipartite était la nécessité de réglementer les politiques de prêt et les taux d'intérêt. En Namibie et en Afrique du Sud, les experts ont plaidé en faveur de réglementations plus strictes en matière de protection des consommateurs. À l'inverse, en Ouganda, les participants ont fait valoir que la loi de 2016 sur les institutions de microfinance et les prêteurs d'argent de niveau 4 devait être révisée en fixant des taux d'intérêt spécifiques aux jeunes pour les établissements de crédit.

\section{(ii) Services financiers informels}

Dans un contexte d'accès limité aux services financiers commerciaux, de nombreux jeunes doivent revenir à l'autofinancement par le biais de plans d'épargne et de formes informelles de prêts (fournis par des membres de la famille ou des réseaux personnels). Dans de nombreux pays, des communautés dynamiques et des organisations de jeunesse ont créé des institutions qui soutiennent l'épargne des jeunes, parfois avec le soutien d'ONG. II existe également des associations d'épargne avec des services connexes fournis par des organisations de jeunesse dont certaines utilisent de l'argent mobile. En Côte d'Ivoire, la Fédération nationale des organisations professionnelles de jeunesse rurale de Côte d'Ivoire (FENOPJERCI) a mis au point un système d'épargne spécifique auquel les membres peuvent contribuer en effectuant des paiements au personnel de la FENOPJERCI ou par le biais de transferts d'argent mobile.

Si ces moyens de financement informels peuvent être très utiles et, en fait, essentiels pour les jeunes entrepreneurs opérant à très petite échelle, l'accélération des affaires commerciales ou la réalisation d'investissements plus coûteux nécessitent toujours l'accès à des prêts plus substantiels. Par conséquent, élargir la gamme de produits et services financiers mis à la disposition des jeunes agri-entrepreneurs au-delà des microcrédits est indispensable pour soutenir la croissance soutenue des jeunes entreprises.

\subsection{Incitations financières: défis, opportunités et bonnes pratiques}

Dans un contexte d'imperfections du marché et de capacités limitées des ONG et des organisations de jeunesse à combler le vide, certains pays ont mis au point plusieurs types d'incitations visant à donner aux jeunes les moyens d'accéder aux services financiers et d'investir dans leurs entreprises. Le type le plus courant d'incitations spécifiques aux jeunes et d'autres incitations accessibles aux jeunes sont les programmes de prêts financés par le gouvernement qui existent principalement dans certains des pays des groupes 2 et 3 - c'est-à-dire les pays à revenu intermédiaire de la tranche inférieure et de la tranche supérieure (Mauritanie, Namibie, Sénégal, Afrique du Sud et Tunisie), ainsi qu'au Mozambique. Certains pays - à l'instar de la Namibie et le Sénégal - ont mis en place des fonds de garantie qui visent à inciter les institutions financières à investir dans la jeunesse en réduisant les risques. S'il n'y a pas d'hypothèse vérifiée empiriquement qui expliquerait l'absence d'un tel mécanisme dans le groupe de pays à faible revenu (groupe 1), on peut supposer que les contraintes financières en sont la cause la plus 
probable ${ }^{6}$. Dans ces pays, il existe des programmes d'incitation financière assortis de délais sous forme de prêts ${ }^{7}$, mais ils ne sont souvent pas intégrés dans le budget ordinaire du gouvernement et dépendent du financement des donateurs. Bien que la pénurie de capitaux soit une contrainte compréhensible, il pourrait s'avérer intéressant de mener une analyse coûts-avantages des bénéfices socio-économiques découlant des programmes d'incitation fiscale existants en les comparant aux incitations financières destinées aux TPEPME. Ces dernières peuvent exiger une épargne publique, mais peuvent aussi être plus efficaces pour autonomiser les jeunes et stimuler la croissance économique locale. Les deux sous-sections suivantes traiteront des prêts et garanties de prêts existants, des défis identifiés et des bonnes pratiques.

\section{(i) Prêts}

Les prêts sont des incitations financières qui peuvent être concessionnelles (fournies à des «conditions plus favorables que les conditions du marché») ou non concessionnelles (basées sur les taux du marché) et peuvent être particulièrement importantes pour soutenir la croissance des petites et moyennes entreprises en l'absence de disponibilité des crédits commerciaux (Tavares-Lehmann, 2017).

Un programme de crédit spécifique aux jeunes qui s'adresse aux jeunes aux tout premiers stades de la planification d'entreprise est le Namibian Youth Credit Scheme (NYCS, Programme de crédits pour la jeunesse namibienne) sous l'égide du Ministère de la jeunesse, des sports et de la culture. NYCS cible tous les jeunes namibiens âgés de 18 à 35 ans et vise à fournir un soutien financier directement aux jeunes qui ne disposent pas de garanties suffisantes pour accéder aux prêts du système bancaire traditionnel. Les prêts proposés vont de 2000 N \$ à 20000 N \$ (environ 100 USD à 1000 USD8). La part des prêts allouée à l'investissement agricole n'a pas encore été annoncée publiquement. NYCS offre en plus du prêt une formation aux bénéficiaires sur les pratiques de gestion de base. Au Mozambique, le Fonds de soutien aux initiatives de la jeunesse (FAIJ) accorde des prêts aux jeunes entrepreneurs (tous secteurs) et a été promu comme une opportunité d'investissement importante pour les IED (investissement étranger direct) dans le guide d'investissement du Mozambique pour les secteurs de l'agro-industrie et de l'industrie légère (APIEX Moçambique, 2018). Cependant, un rapport d'évaluation révèle que le gouvernement a du mal à se faire rembourser les prêts par les bénéficiaires et le non-remboursement demeure un problème majeur (eMJee Consult, 2018). Par ailleurs, des discussions de groupe avec des jeunes menées au Mozambique par le National Democratic Institute (NDI) et le Centre de recherche sur la gouvernance et le développement (CPGD) ont conclu que le suivi du renforcement des capacités et de l'assistance technique était limité une fois que les fonds étaient décaissés (NDI et CPGD, 2016). Bien qu'il soit impossible d'établir une relation de cause à effet entre ces deux

\footnotetext{
6 Tavares-Lehmann (2017) conclut que «très souvent, dans les économies en développement, elles [les incitations fiscales] sont le seul type d'incitation offert, car ces pays n'ont pas les ressources financières pour fournir des incitations financières directes pratiquées par leurs homologues développés.

7 Un cas est celui de l'Initiative conjointe FAO-Ministère de l'Agriculture, de l'Industrie animale et de la pêche Youth Inspiring Youth in Agriculture Initiative (Les jeunes motivent les jeunes dans l'agriculture) en Ouganda, qui a identifié des jeunes champions qui ont reçu de petites subventions pour leurs entreprises.

${ }^{8}$ Cela correspond à la taille habituelle des tickets de prêts de microfinance en Namibie (Bank of Namibia Research Department, 2004).
} 
observations, elles indiquent néanmoins la nécessité de fournir des ensembles complets d'incitations combinant différents types de services, comme nous le verrons plus loin.

Cependant, il existe peu de prêts spécifiques qui soutiennent les jeunes dans l'agriculture et qui s'attaquent à certains des problèmes spécifiques auxquels ces jeunes sont confrontés. Par contre, c'est un fait reconnu qu'un ciblage plus spécifique prenant en compte les contraintes des jeunes entrepreneurs investissant le long des filières agricoles, ainsi que le retour sur investissement en termes de potentiel de développement économique, est nécessaire et constitue une priorité récurrente. À cet égard, les prêts fonciers tunisiens fournissent l'exemple d'une bonne pratique intéressante, car l'accès à ces prêts est conditionné par le respect de critères spécifiques (voir encadré ci-dessous).

\section{Encadré 2: Les prêts fonciers tunisiens}

Comme dans de nombreux pays, le manque d'accès à la terre est un obstacle important à l'entrée des jeunes agri-entrepreneurs en Tunisie. Afin de relever ce défi, l'Agence tunisienne de promotion des investissements agricoles (APIA) a mis au point un dispositif de crédit spécifique destiné à aider les jeunes agri-entrepreneurs à acquérir et exploiter des terres.

Les prêts fonciers sont un instrument spécifique du code des investissements tunisien et sont accessibles à tous les jeunes de moins de 40 ans diplômés de l'université (en agriculture et domaines techniques connexes) ou titulaires d'un certificat de compétence professionnelle. La terre elle-même doit avoir un potentiel qui n'est actuellement pas pleinement exploité.

Le volume total de chaque prêt peut atteindre jusqu'à 250000 DT (environ 85000 USD), avec un taux d'intérêt de 3 pour cent, un délai de grâce de 7 ans et une exigence d'autofinancement de 5 pour cent pour l'acquisition de terrains et de 10 pour cent pour le développement foncier (y compris l'électrification).

Les jeunes agri-entrepreneurs qui ont bénéficié des prêts fonciers sont également éligibles à d'autres subventions d'investissement non spécifiques aux jeunes (subventions complétant les investissements privés), telles que celles qui couvrent une partie des dépenses liées aux investissements dans les technologies permettant d'économiser l'eau.

Si les modalités opérationnelles (y compris les critères d'éligibilité) des prêts fonciers ont varié dans le temps, ce dispositif de crédit assez bien établi est accompagné de données sur le montant total des prêts qui sont collectées depuis 1985. Le volume total des nouveaux prêts fonciers n'a cessé d'augmenter au cours des dernières années.

Sources:

APIA. Site Web. http://www.apia.com.tn/prets-fonciers.html; http://www.apia.com.tn/prets-fonciers-2.html FAO. 2013. Tunisie: Financement du secteur agricole. Rome, FAO, pp. 100-101.

FAO and INRAT. 2020. Dynamique de l'investissement dans le système agricole tunisien et perspectives de développement des investissements par et pour les jeunes. Rome. http://www.fao.org/documents/card/en/c/cb0563fr

http://www.onagri.tn/uploads/veille/Impact-de-la-loi-sur-les-investissements-apia-dgfiop.pdf 
Enfin, il existe une série de mécanismes et de programmes d'incitation financière détenus, contrôlés ou gérés par le gouvernement qui ciblent sans distinction toutes sortes de petits producteurs, y compris les jeunes et les femmes. Même si ces programmes ne sont pas spécifiques aux jeunes, certaines bonnes pratiques utiles et des enseignements relatifs aux risques éventuels peuvent en découler. En Afrique du Sud, il existe une pléthore de programmes gérés ou financés par le gouvernement (qui ne ciblent pas spécifiquement les jeunes, mais leur sont néanmoins accessibles), notamment les institutions financières micro-agricoles d'Afrique du Sud (MAFISA) qui ont pour objectif spécifique de répondre aux besoins financiers des petits exploitants grâce à l'octroi de prêts à des taux d'intérêt bas (plafonnés à 8 pour cent). Les prêts MAFISA sont acheminés par l'intermédiaire de la Banque foncière vers des intermédiaires financiers sélectionnés qui octroient de petits prêts. L'objectif du gouvernement était de créer des synergies entre la MAFISA et les services de vulgarisation du gouvernement, qui soutiendraient à la fois les producteurs pour leur demande de prêt et fourniraient une assistance technique une fois le prêt accordé pour en assurer la viabilité économique. Malgré l'excellente structure de ce système, un examen indépendant des performances et des dépenses commandé par le Gouvernement sud-africain a identifié une série de défis (Cornerstone Economic Research (Pty) Ltd., 2014). Premièrement, malgré l'intégration prévue de MAFISA avec les services de vulgarisation, le soutien de ces derniers semble limité. Deuxièmement, I'accès géographique aux fonds MAFISA était inégal. Troisièmement, le modèle économique aurait pu être amélioré, car il était trop risqué pour les intermédiaires et ne pouvait pas être rentable pour ceux-ci au cas où les taux de défaut dépasseraient $5 \%$. Notez également que les taux d'intérêt établis par MAFISA étaient fixes, alors que les taux d'intérêt généraux sont déterminés par le marché et donc sujets à changement.

En Mauritanie, une institution de microfinance spécifique - I'Union nationale des mutuelles d'investissement et de crédit oasien et des zones pluviales (UNMICO) — accorde des prêts au secteur agricole. L'UNMICO a le mérite de combiner un crédit rural axé sur les besoins à des taux d'intérêt bas avec un soutien au développement des capacités pour assurer la viabilité économique d'un investissement, ce qui est important (comme on le verra plus loin). L'encadré ci-dessous fournit des informations plus détaillées sur les modalités opérationnelles de I'UNMICO.

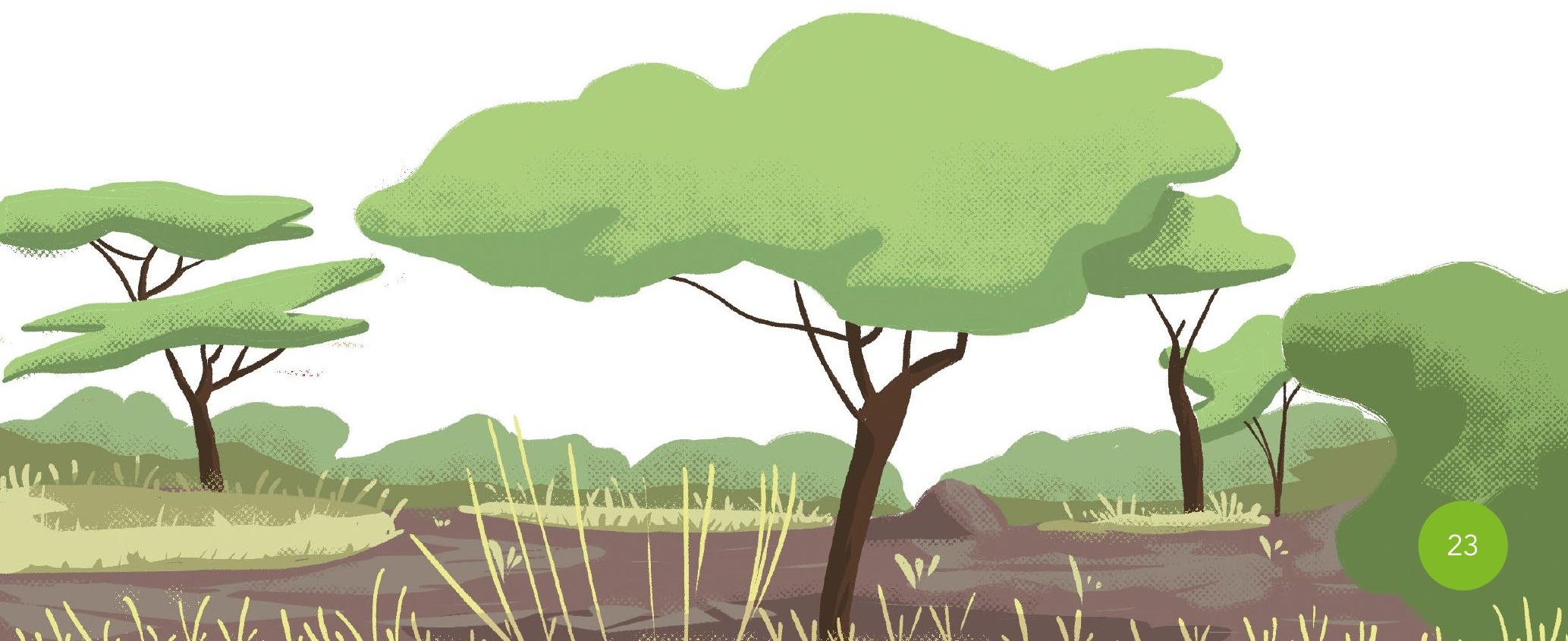




\section{Encadré 3: Le modèle économique de l'Union Nationale des Mutuelles d'Investissement et de Crédit oasien et des Zones Pluviales (UNMICO)}

L'UNMICO gère des caisses locales (fonds avec une caisse spécifique) en partenariat avec des agents économiques locaux (coopératives, organisations de producteurs, agriculteurs et autres) qui deviendront copropriétaires («sociétaires») de la caisse et devront augmenter progressivement leurs responsabilités managériales et financières. L'objectif principal des caisses est l'octroi de microcrédits à court terme qui constituent $80 \%$ du portefeuille de prêts.

Préalablement à la création d'une caisse, l'UNMICO réalise des études de faisabilité et collabore avec les acteurs locaux pour identifier et donner la priorité aux enjeux clés, ainsi que leurs solutions potentielles en fonction d'un ensemble de critères économiques, techniques et socioculturels.

Par la suite, l'UNMICO offre une formation aux sociétaires et aux bénéficiaires sur diverses questions, dont les connaissances et la gestion financières, les pratiques comptables ou la gestion et la gouvernance d'une caisse et d'autres organisations. L'UNMICO assure également un suivi et un contrôle réguliers avec chaque caisse et avec les bénéficiaires pour contribuer à la pérennité de chaque projet.

Sources:

UNMICO. Note sur l'accompagnement des bénéficiaires.

Statistiques les plus récentes sur chaque caisse: http://unmico.net/?q=node/33

\section{(ii) Garanties de prêt}

Dans les pays où les institutions financières sont assez bien développées et représentées dans les zones rurales, les fonds de garantie de prêts peuvent être un outil puissant pour inciter ces institutions à prêter à des clients qui peuvent être perçus comme risqués, comme les jeunes et les femmes, et qui peuvent n'avoir aucune garantie. Au Sénégal, le Fonds de garantie des investissements prioritaires (FONGIP) offre des garanties de prêts avec des dispositifs spécifiques ciblant les jeunes et les femmes, à la fois individuellement et aux groupes de jeunes/femmes, et pour soutenir des activités indépendantes de jeunes (Sénégal - Ministère de l'Économie, des Finances et du Plan, sans date). Les garanties peuvent être fournies tant aux candidats individuels (avec une demande officielle signée par l'institution financière), qu'aux institutions financières pour les portefeuilles de prêts (FONGIP, sans date). Les garanties couvrent les prêts d'une durée maximale de cinq ans, avec un plafond maximal de 70 pour cent des prêts impayés. FONGIP facture des frais de commission allant jusqu'à 1,5 pour cent pour la fourniture de la garantie (ADIE, non daté). En Namibie, la Bank Windhoek a mis en place des systèmes de garantie avec le Conseil national de la jeunesse et la Fondation Erongo pour le développement, où cette 
dernière fournit des garanties pour 60 pour cent du financement des petites et moyennes entreprises (PME) qui ne peuvent fournir aucune forme de garantie. En Tunisie, où un tel fonds de garantie n'existe pas encore, sa mise en place est devenue l'une des priorités clés de la feuille de route élaborée conjointement par I'APIA, la FAO et I'INRAT dans le cadre du processus de planification stratégique.

\subsection{Recommandations politiques: améliorer l'accès aux services financiers}

Les gouvernements peuvent apporter une contribution réellement importante à l'autonomisation des jeunes agri-entrepreneurs, en leur permettant d'investir à travers des incitations financières. Cependant, plusieurs difficultés subsistent. Ces problèmes pourraient être surmontés en considérant les cinq recommandations suivantes: (i) réduire les asymétries d'information et améliorer la communication entre les agences offrant des incitations et les jeunes bénéficiaires; (ii) améliorer les critères de ciblage des programmes d'incitation financière; (iii) favoriser les synergies avec d'autres programmes dirigés par des gouvernements et des organisations non gouvernementales; (iv) suivre la performance des dispositifs $d$ 'incitation financière; et ( $v$ ) envisager des mécanismes innovants pour améliorer l'accès aux services financiers commerciaux.

Améliorer la communication et réduire les asymétries d'information. Lorsque les gouvernements fournissent des incitations financières, ils doivent informer le public de leur existence et lui expliquer comment ces mesures d'encouragement fonctionnent en recourant à tous les canaux de communication possibles (radio, affiches et réseaux sociaux entre autres) pour sensibiliser les bénéficiaires potentiels. Il est tout aussi important que les règles et les critères de sélection soient clairs et les procédures aussi simples que possible. Les différentes analyses et évaluations ont révélé que de nombreux bénéficiaires potentiels n'étaient pas au courant des incitations mises en place ou ne comprenaient pas les modalités et les procédures de candidature. Cela peut conduire à des asymétries d'information qui favorisent les jeunes diplômés de l'enseignement supérieur, qui ont accès à Internet et/ou aux sources gouvernementales. Comme l'a fait valoir une étude de fond, dans les conditions actuelles, cette distorsion peut avantager de manière disproportionnée les jeunes qui «ont accès à l'information [...] et utilisent avec succès toutes ces opportunités aux dépens de la majorité des jeunes défavorisés et de leurs organisations qui sont abandonnés dans les zones rurales pauvres» (Hakizimana, à paraître).

Définir des critères de ciblage spécifiques pour les bénéficiaires des mesures d'incitation. Il est essentiel d'établir des critères de ciblage clairs déterminant qui doit bénéficier des incitations financières. Cela n'inclut pas seulement des critères spécifiques aux jeunes, mais aussi des critères définissant les sous-groupes prioritaires parmi les jeunes (instruits vs non éduqués, hommes vs femmes, ruraux vs urbains, etc.). Les jeunes ne constituent pas un groupe homogène et les besoins, les capacités et les contraintes, par exemple, d'un jeune diplômé urbain et d'un agriculteur rural ayant une éducation de base varient considérablement. De nombreuses incitations financières ciblent en effet les jeunes de niveau supérieur (critère inscrit dans les critères d'éligibilité, comme en Tunisie, ou plus implicitement, à travers des procédures qui excluent de facto les 
jeunes moins scolarisés). Des critères d'éligibilité stricts ciblant les jeunes diplômés de l'enseignement supérieur peuvent être parfaitement justifiés dans des pays comme la Tunisie, étant donné que les taux de chômage des jeunes diplômés de l'enseignement supérieur sont considérablement plus élevés $(28,1$ pour cent) que le taux de chômage global des jeunes (25,5 pour cent, voir: FAO et INRAT, 2020b) et que le ciblage des diplômés universitaires peut aider à utiliser des ressources rares de manière plus efficace et durable. Inversement, ces critères peuvent être contre-productifs dans les pays où les tendances socio-économiques indiquent des priorités différentes. Aussi est-il important de souligner que les critères d'éligibilité étroits des prêts fonciers, limitant l'accès aux seuls jeunes diplômés en agriculture, agronomie ou disciplines connexes, ont été remis en question par certains jeunes diplômés d'autres disciplines (économie, marketing, etc.) qui avaient pourtant acquis des compétences agricoles pratiques dans les exploitations familiales de leurs parents.

Favoriser les synergies avec d'autres programmes gouvernementaux et non gouvernementaux. Si les prêts à conditions avantageuses (taux d'intérêt bas, délais de grâce prolongés, etc.) peuvent être des incitations utiles pour les jeunes agrientrepreneurs à investir dans leur entreprise, l'efficacité des dépenses publiques dans ce domaine pourrait être considérablement accrue si les prêts étaient consentis en synergie avec d'autres programmes gouvernementaux et non gouvernementaux. En particulier, des services complémentaires de renforcement des capacités, de marketing (par exemple à travers l'accès aux foires) et de préparation aux affaires seraient essentiels pour garantir que les jeunes agri-entrepreneurs aient non seulement accès aux services financiers, mais soient également habilités à améliorer la productivité et l'efficacité de leurs entreprises. Le module complet fourni en 2019 à 121 jeunes par le Ministère namibien des Sports, de la Jeunesse et des Services nationaux, qui comprend un financement, la formation, l'encadrement et le mentorat fournit un exemple de programme qui vise à résoudre ce problème. Le mécanisme de financement destiné aux 121 entreprises de jeunesse des circonscriptions est hébergé par le Ministère des Sports, de la Jeunesse et du Service national en collaboration avec la Banque de développement de Namibie (DBN). Les bénéficiaires n'ont pas besoin de fournir une garantie, car celle-ci est fournie par le biais d'un système de garantie de crédit, en collaboration avec l'Association namibienne des risques spéciaux (Amadhila, non publié).

Surveiller la performance des programmes d'incitation financière. La divulgation publique de la viabilité financière des systèmes de prêt, ainsi qu'un suivi plus cohérent des performances des bénéficiaires peuvent contribuer de manière importante à accroître la compréhension collective de ce qui fonctionne et de ce qui ne fonctionne pas en termes d'incitations financières. Cependant, il existe peu de preuves d'un suivi et d'une évaluation systématiques de la performance des incitations financières. En fait, à l'exception de l'Afrique du Sud, l'auteur n'a pu recueillir aucune information accessible au public concernant les évaluations commandées par les gouvernements. II semble y avoir des évaluations de programmes de prêts financés par des partenaires tiers, tels que des organisations de coopération technique et financière et des ONG (comme c'est le cas pour le Mozambique). Il en découle que les connaissances sur la performance des 
programmes de crédit, sur le taux de remboursement, sur l'impact réel des prêts sur la performance des jeunes agro-entrepreneurs sont limitées.

Envisager des mécanismes innovants pour améliorer l'accès aux services financiers commerciaux. La fixation de taux d'intérêt spécifiques aux jeunes pour les banques commerciales peut être une idée séduisante, mais ne peut en fait que dissuader davantage les banques de prêter aux jeunes en l'absence de garanties supplémentaires. Au-delà des garanties de prêt, les gouvernements peuvent envisager toute une gamme de mécanismes permettant aux jeunes d'accéder aux services financiers commerciaux. La Tunisie a récemment adopté une loi selon laquelle l'État s'engage à payer la différence entre le taux d'intérêt d'un prêt et le taux moyen du marché monétaire jusqu'à 3 points pour les investissements dans des secteurs spécifiques, dont l'agriculture (Journal officiel de la République tunisienne, 2019). Un autre mécanisme innovant pouvant accroître l'accès au financement commercial qui devrait être examiné avec attention est le financement mixte, à savoir «l'utilisation stratégique du financement du développement pour la mobilisation de financements supplémentaires en faveur du développement durable (OCDE, 2018)».

\section{Accès à la terre}

Après le capital financier, la terre demeure le facteur de production le plus important pour les jeunes agri-entrepreneurs. Les jeunes peuvent accéder à la terre par héritage, distribution gouvernementale ou par le biais du marché (Comité technique foncier et développement, 2020). Au cours des dernières décennies, l'accès à la terre est devenu de plus en plus difficile pour les jeunes et a été l'un des défis les plus fréquemment identifiés empêchant les jeunes d'investir dans l'agriculture (voir Figure 9). Dans les pays où un système de notation a été utilisé pour évaluer l'accès des jeunes agri-entrepreneurs et agriculteurs à la terre (en utilisant une fourchette comprise entre 0 (pas d'accès) et 5 (excellent accès), les réponses étaient systématiquement à 1, sauf au Mali (en raison d'une intervention gouvernementale ciblée qui sera discutée dans ce chapitre) et en Tunisie (en raison des prêts fonciers).

Figure 9: Accès des jeunes à la terre tel qu'identifié dans les processus d'évaluation et de planification ( 0 = pas d'accès; 5 = excellent accès)

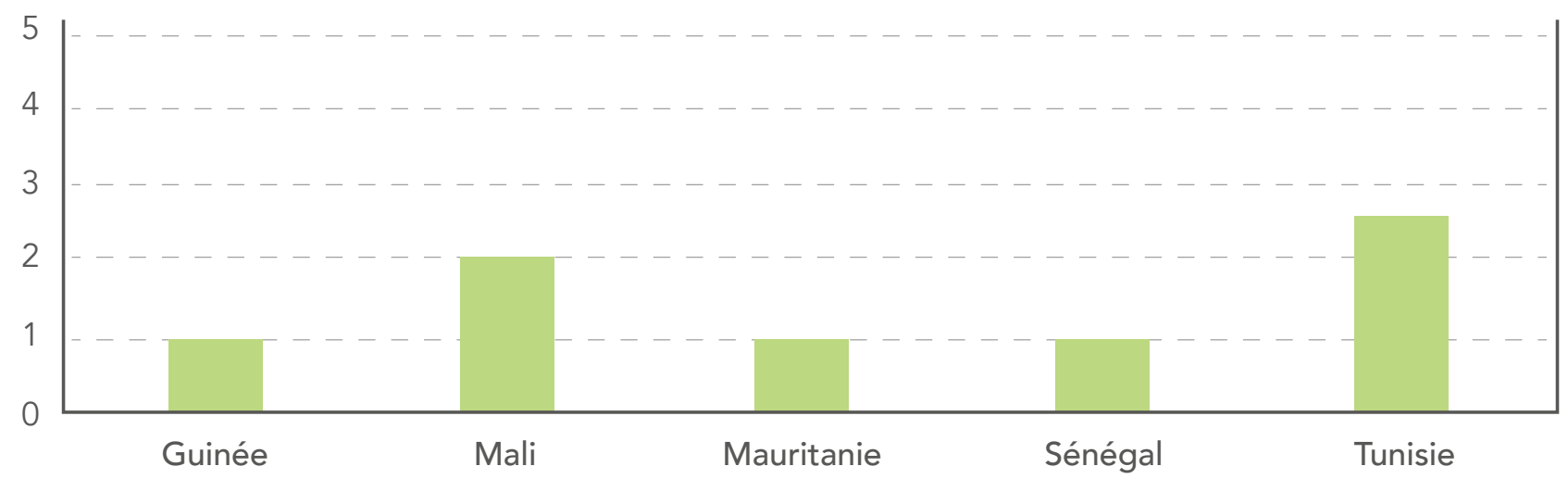

Source: Figure élaboré par l'auteur à partir de la base de données FAO RAl et des évaluations des capacités des jeunes, 2020. 
Les sections suivantes (1) discuteront des principaux défis, opportunités et bonnes pratiques concernant l'accès à la terre par (i) héritage, (ii) acquisition directe ou location, et (iii) distribution; et (2) formuleront des recommandations politiques fondées sur cette analyse.

\subsection{Accès des jeunes à la terre par le biais d'un l'héritage, du marché et de programmes de distribution}

\section{(i) Accès à la terre par héritage}

Traditionnellement, les jeunes héritaient des terres de leurs parents. Dans un contexte de transition démographique qui se caractérise par une espérance de vie accrue et des taux de fécondité qui diminuent lentement, mais qui sont toujours élevés, les jeunes sont moins susceptibles d'accéder à la terre par héritage. À mesure que les taux d'espérance de vie augmentent, les parents sont «moins susceptibles de céder des terres à leurs enfants lorsqu'ils [ils] entrent sur le marché du travail (FIDA, 2019)». Dans le même temps, la croissance démographique conduit à une densité accrue dans les zones rurales, ce qui réduit à son tour la disponibilité des terres et augmente leur valeur. Par conséquent, dans de nombreux cas, les jeunes «ne bénéficient que de droits fonciers subsidiaires et travaillent sur les terres familiales pour une rémunération faible ou nulle (FAO, CTA et FIDA, 2014)».

Pour les jeunes femmes, il est souvent pratiquement impossible d'accéder à la terre par héritage, car de nombreux systèmes coutumiers favorisent la succession agricole au fils aîné (FAO, CTA et FIDA, 2014). Plusieurs pays ont souligné les obstacles liés aux sexospécifités en ce qui concerne les droits fonciers communaux qui favorisent généralement les jeunes hommes au détriment de leurs sœurs?. En Namibie, les participants à l'évaluation des capacités ont donc spécifiquement suggéré de réviser la section sur l'héritage des terres communales de la loi sur la réforme foncière communale pour l'aligner sur le $3 e$ principe CFS RAI traitant de l'égalité des sexes.

\section{(ii) Accès à la terre par le biais du marché}

Les jeunes peuvent également accéder à la terre par le biais du marché, soit par créditbail ou location - qui peut dans de nombreux cas être la seule option abordable pour les jeunes agriculteurs - ou par achat pur et simple. Compte tenu de la rareté croissante des terres arables, l'acquisition de terres peut dans de nombreux cas être trop onéreuse pour les jeunes, surtout en l'absence de services financiers et d'incitations telles que les prêts fonciers tunisiens.

Les gouvernements peuvent contribuer à l'efficacité des marchés fonciers et veiller à ce que les jeunes soient habilités à s'engager sur ces marchés grâce à des lois et réglementations solides. Dans plusieurs pays, les jeunes femmes et hommes participant aux évaluations ont fait valoir que les processus d'acquisition ou de location de terres peuvent être confus, lourds ou coûteux et dissuader les jeunes de s'engager dans l'acquisition ou la location

${ }^{9}$ Obstacles mis en évidence, entre autres, dans les documents d'information rédigés par Kama Berté (Côte d'Ivoire) et Alina Amadhila (Namibie). 
de terres. Même si ces problèmes ont été moins fréquemment mis en évidence que les autres examinés dans ce document, il convient de noter que des solutions concrètes inscrites dans les stratégies politiques existent.

En Côte d'Ivoire, où de tels problèmes ont été signalés, le 2e Plan national d'investissement vise à i) sensibiliser les jeunes aux procédures existantes; et ii) sécuriser les droits fonciers coutumiers à faible coût par le biais de demandes collectives (Côte d'lvoire, 2017). Des mesures supplémentaires pourraient inclure un processus d'acquisition de terrains plus simple et moins coûteux (par exemple en fixant un plafond pour les frais administratifs), ainsi qu'un statut juridique amélioré pour les contrats de location foncière.

\section{(iii) Accès à la terre grâce à des réformes et des programmes redistributifs}

Les réformes et programmes de redistribution peuvent «faciliter un accès étendu et équitable à la terre et un développement rural inclusif (FAO, 2012 c)». Parmi ceux-ci il faut citer: «l'attribution de terres publiques, des mécanismes volontaires et fondés sur le marché ainsi que l'expropriation de terres privées, de pêcheries ou de forêts à des fins publiques (FAO, 2012 c)». L'une des principales conclusions des processus d'évaluation est que les réformes et programmes de redistribution pourraient constituer un outil utile pour faire progresser la propriété foncière chez les jeunes. Cependant, ils doivent être bien réglementés et orientés vers l'autonomisation des jeunes et être idéalement combinés avec d'autres interventions de renforcement des capacités.

Récemment, certains pays ont progressé dans la mise en place d'un quota spécifique pour les jeunes. Par exemple, la loi malienne sur le régime foncier réserve au moins 15 pour cent de toutes les terres défrichées par le gouvernement aux jeunes et aux groupes de femmes (Journal officiel de la République du Mali, 2017). Si le quota peut faire l'objet de discussions, le simple fait que les terres ne soient pas uniquement attribuées aux seuls chefs de ménage peut être considéré comme un progrès significatif. II convient de noter que cette loi a été élaborée avec la forte implication de la société civile et des organisations de producteurs, y compris l'organisation faîtière des jeunes ruraux du Mali, la Fédération nationale des jeunes ruraux (FENAJER).

En Afrique du Sud, le projet de Politique de sélection des bénéficiaires et d'attribution des terres fixe un objectif encore plus ambitieux et propose que «pas moins de $50 \%$ des allocations de terres agricoles aux petits exploitants dans le cadre du programme de redistribution [soient alloués] aux femmes, $40 \%$ aux jeunes, et $10 \%$ aux personnes handicapées (Afrique du Sud, 2020)». Cependant, l'établissement d'un équilibre socialement acceptable entre une allocation de ressources visant à stimuler la productivité et les interventions visant à soutenir les segments les plus vulnérables des groupes historiquement défavorisés semble soulever des problèmes. Cette orientation politique donnant la priorité aux "diplômés agricoles sans emploi (Afrique du Sud, 2020)» peut être justifiée en termes d'allocation efficace des ressources publiques pour stimuler la croissance et est alignée sur l'objectif de la politique de «raviver la classe des agriculteurs commerciaux noirs». Pourtant, compte tenu de ses objectifs supplémentaires de «répondre à des besoins divers» et de cibler les populations vulnérables, y compris les 
ruraux pauvres, il sera important d'observer comment et si la politique sera également à même de promouvoir l'inclusion économique des jeunes les moins privilégiés (Hakizimana, publication imminente).

À un autre niveau, il est essentiel de s'assurer que les jeunes seront dotés des compétences nécessaires pour cultiver de manière productive les terres qui leur sont distribuées. À cet égard, il est à noter que la feuille de route RAI tunisienne appelle à la distribution des terres aux pasteurs, tout en soulignant que «l'attribution des terres domaniales aux jeunes doit être la plus encadrée possible avec à la fois un accès à la formation et au financement, pour éviter les écueils des expériences précédentes (FAO et INRAT, 2020b)».

\subsection{Recommandations politiques: améliorer l'accès à la terre}

Le manque d'accès à la terre reste un défi majeur pour les jeunes femmes et hommes. Certains des principaux défis auxquels les jeunes sont confrontés en matière d'accès à la terre pourraient être surmontés en considérant les cinq recommandations suivantes: (i) Éliminer toute discrimination fondée sur le sexe dans les lois et pratiques en matière d'héritage; (ii) veiller à ce que les réglementations et procédures d'acquisition et de location de terrains soient claires, rentables et bien connues; (iii) établir des critères de ciblage clairs et spécifiques aux jeunes pour les programmes de distribution des terres; et (iv) associer la répartition des terres avec des mécanismes de soutien supplémentaires.

Éliminer toute discrimination fondée sur le sexe dans les lois et pratiques en matière de succession. Pour les jeunes femmes, il est souvent pratiquement impossible d'accéder à la terre par héritage, car de nombreux systèmes coutumiers favorisent la succession agricole au fils aîné. Les gouvernements peuvent contribuer à l'autonomisation des jeunes en éliminant la discrimination fondée sur le sexe dans les régimes d’héritage coutumier et légal.

Veiller à ce que les réglementations et les procédures d'acquisition et de location de terres soient claires, rentables et bien connues. Lorsque les procédures d'acquisition et de location de terres sont confuses, lourdes ou coûteuses, les moins instruits et les moins expérimentés s'abstiendront de s'engager dans des transferts de terres ou seront désavantagés en raison du manque de connaissance de leurs droits et responsabilités. Faire en sorte que les réglementations et les procédures soient claires et facilement compréhensibles, notamment en les traduisant dans les langues locales, peut autonomiser les communautés rurales en général, et les jeunes en particulier. En outre, des campagnes de communication appropriées et bien ciblées, utilisant les canaux et le langage préférés des jeunes, contribuent à sensibiliser ces derniers à ces réglementations.

Établir des critères de ciblage clairs et spécifiques aux jeunes pour les programmes de distribution des terres. Dans le contexte des programmes de distribution des terres, les bénéficiaires doivent dès le départ être clairement désignés. L'établissement de critères de ciblage clairs et spécifiques aux jeunes, de préférence très précis, est essentiel à cet égard. Il peut être utile de sélectionner les bénéficiaires par le biais d'un processus concurrentiel basé sur des propositions commerciales soumises par les candidats dans le but de garantir la viabilité économique du programme de distribution. 
Associer la distribution des terres à des mécanismes de soutien supplémentaires. Lorsque la terre est distribuée aux bénéficiaires qui ne possèdent pas les bonnes compétences agricoles et commerciales, le programme peut échouer d'un point de vue économique. Par conséquent, il est essentiel de fournir des mécanismes de soutien supplémentaires, notamment des services de vulgarisation, des formations commerciales et de gestion, des services financiers et éventuellement des contributions en nature telles que des équipements de base.

\section{Information et services techniques}

Alors que l'accès aux services financiers est une condition préalable pour permettre aux jeunes d'investir dans l'agriculture et les systèmes alimentaires, les jeunes agrientrepreneurs ont généralement besoin de services supplémentaires pour créer des entreprises florissantes. Cela comprend les incubateurs, l'encadrement et d'autres services de conseil qui aident les jeunes à développer, piloter et mettre en œuvre des projets économiquement viables. L'accès à l'information et aux services techniques est comparativement plus élevé que l'accès aux facteurs de production, et en particulier en ce qui concerne les services liés au développement des entreprises (voir le Figure 10 cidessous). Dans les pays où un système de notation a été utilisé pour évaluer l'accès des jeunes agri-entrepreneurs et agriculteurs à l'information et aux services techniques (en utilisant une fourchette comprise entre 0 (pas d'accès) et 5 (excellent accès), les services de développement d'entreprise et d'incubation ont été considérés relativement accessibles (3/5 en Guinée et en Mauritanie [cette dernière pour le développement des affaires uniquement]) ou très accessibles (4/5 au Sénégal et en Tunisie). L'accès aux services de vulgarisation a été considéré comme étant plus faible (entre 1 en Guinée et au Sénégal, 2 au Mali et en Mauritanie et 2,5 en Tunisie).

Figure 10 : Accès à l'information et aux services techniques tels qu'identifiés dans les processus d'évaluation et de planification ( $0=$ pas d'accès; $5=$ excellent accès $)$

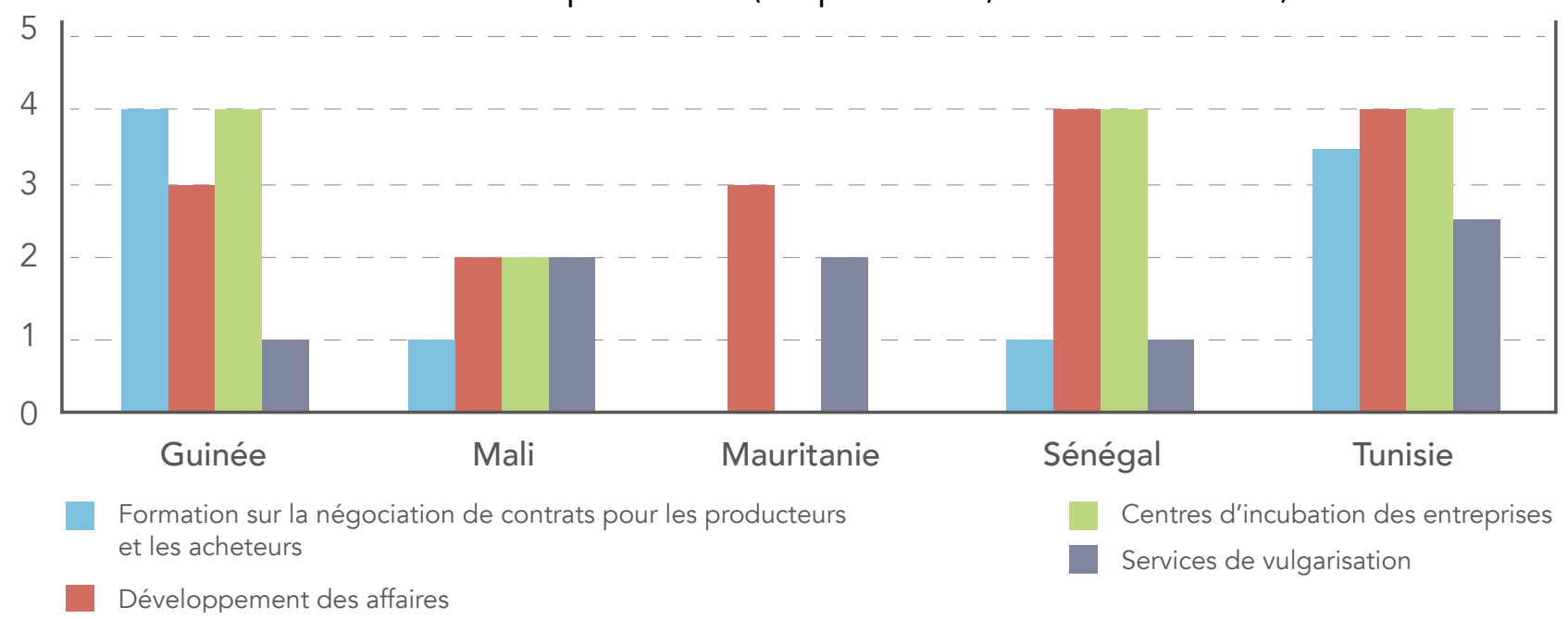

Source: Figure élaboré par l'auteur à partir de la base de données FAO RAl et des évaluations des capacités des jeunes, 2020. 


\subsection{Accès aux incubateurs, aux services d'encadrement et de conseil}

Les incubateurs, l'encadrement et autres services de conseil font partie des mesures d'incitation liées aux «informations et services techniques» (Tavares-Lehmann, 2017). Un incubateur d'entreprises est une «organisation qui accélère et systématise le processus de création d'entreprises prospères en leur fournissant une gamme complète et intégrée de soutien, notamment un espace d'incubation, des services de soutien aux entreprises et des opportunités de regroupement et de mise en réseau (Commission européenne, 2002)». Les services de soutien aux entreprises peuvent inclure la fourniture d'études prospectives, notamment l'identification des opportunités de marché, le soutien au marketing et le mentorat. II existe des preuves empiriques de la «relation entre l'incubation d'entreprises et la réalisation des objectifs liés au capital humain, à l'emploi et à la croissance (Fernández Fernández, Blanco Jiménez et Cuadrado Roura, 2015)».

Les pépinières d'entreprises peuvent, mais ne doivent pas nécessairement être détenues par l'État. Elles peuvent être gérées par des agences gouvernementales, des universités, des ONG ou des entreprises privées avec un financement provenant de ressources publiques, privées et de bailleurs de fonds. Ces centres d'incubation sont généralement gratuits. Dans la plupart des pays du groupe 1 et du groupe 2, les pépinières d'entreprises et autres services d'information et de formation sont en fait fournis par des ONG et des fondations. Cela vaut par exemple pour la Côte d'Ivoire, la Guinée, le Mozambique et I'Ouganda. La Guinée compte une pléthore d'incubateurs dirigés par des ONG, tels que Saboutech et Osez Innover. Saboutech est une initiative menée par le secteur privé avec un financement d'entreprises multinationales et du gouvernement (Forbes Afrique, 2016). Au Sénégal, Yeesal Agri Hub est une association dirigée par ses membres à qui elle demande des frais minimes et qui a reçu le soutien de l'ONG italienne LVIA.

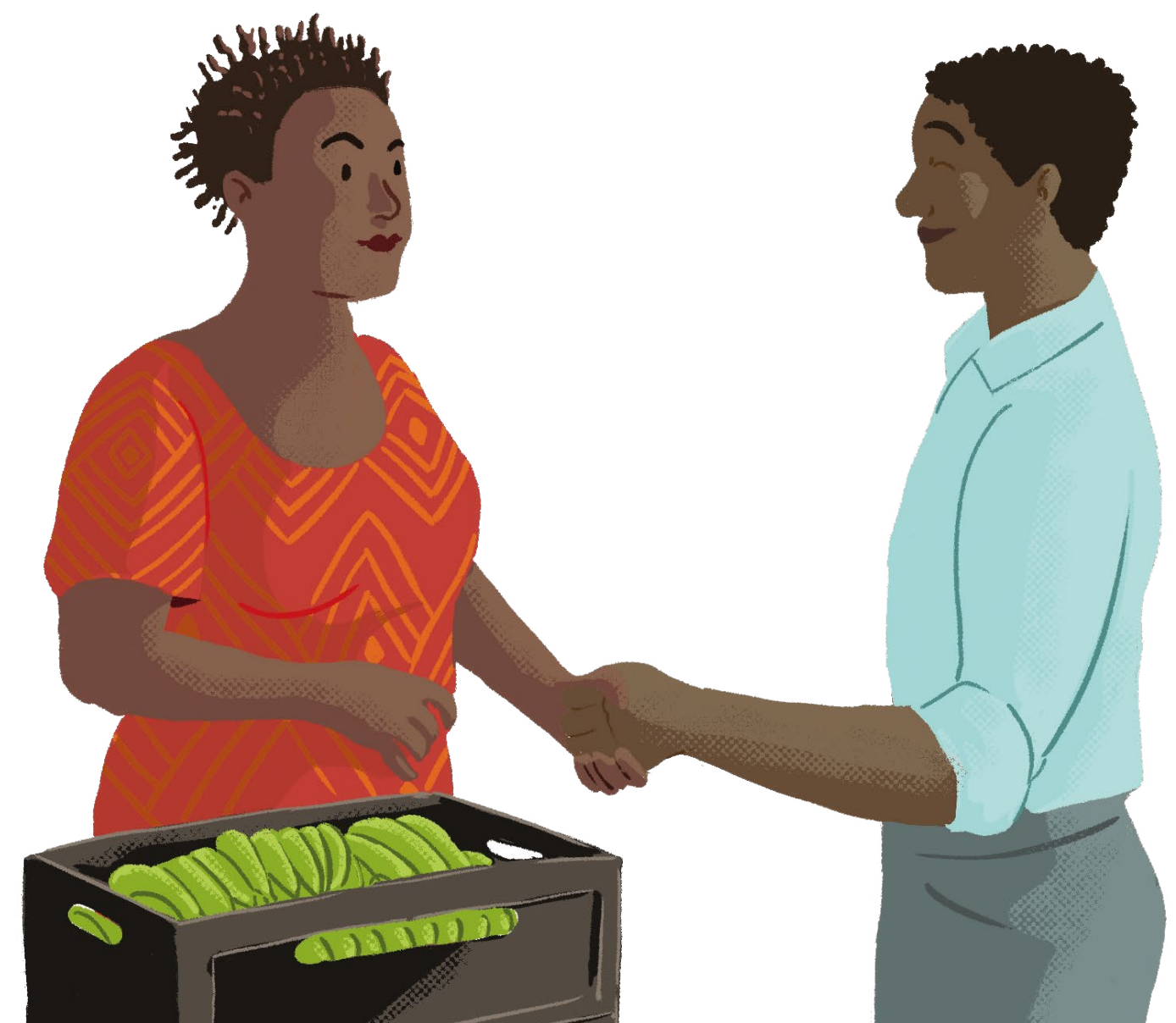


Dans d'autres cas encore, les incubateurs d'entreprises sont dirigés par des partenariats entre le secteur public, les donateurs et les partenaires techniques. Au Sénégal, le Modèle d'insertion des jeunes dans l'agriculture (MIJA) est géré par l'Agence Nationale pour la Promotion de l'Emploi des Jeunes (ANPEJ) et est financé par le gouvernement avec le soutien de la FAO et l'Agence espagnole de coopération au développement. Les six plates-formes MIJA offrent des services d'incubation complets, avec des fermes modèles. L'un de ses résultats clés est l'autonomisation des jeunes agri-entrepreneurs qui ont réussi à s'organiser au sein de l'association d'entraide Réseau africain pour la promotion de l'entrepreneuriat agricole (RAPEA), qui fournit des services de soutien technique aux start-up des agri-entrepreneurs, favorise le partage des connaissances et facilite l'action collective.

Dans d'autres cas encore, les incubateurs peuvent être gérés par des agences gouvernementales, éventuellement en collaboration avec des universités locales. En Tunisie, les Pépinières d'entreprises agricoles sont gérées par l'Agence de promotion des investissements agricoles (APIA), souvent en collaboration avec des universités (APIA, 2020). Ces pépinières proposent des espaces de bureaux, des parcelles pilotes expérimentales, des formations et un encadrement. 100 chercheurs universitaires, 50 formateurs techniques, 33 collaborateurs bancaires et 20 spécialistes de l'économie et de la finance accompagnent les jeunes agri-entrepreneurs dans le développement et le raffinement de leurs projets et améliorent leurs compétences en gestion sur une période de 24 mois. Par la suite, APIA aide les jeunes qui ont participé à la pépinière à commercialiser leurs produits en les faisant participer à des foires et événements publics, et à trouver des ressources financières, notamment en accédant aux subventions d'investissement d'APIA et autres partenariats avec la BTS et d'autres banques. Même au-delà de la période de mentorat de 24 mois, les jeunes qui ont suivi préalablement la formation peuvent revenir sur les lieux pour échanger et créer des réseaux avec leurs pairs. En termes d'accès au financement, plus de 100 projets ont été financés par BTS et d'autres projets à travers des partenariats avec l'APIA entre 2009 et 2016. Malgré ces efforts, les difficultés n'ont pas disparu. À savoir, les avantages offerts par le gouvernement ne sont pas toujours connus. Le renforcement de la coordination avec d'autres mécanismes d'appui financés par les donateurs, les coopératives et le secteur privé constitue un autre défi, comme le confirment les conclusions des travaux d'évaluation de la FAO et de I'INRAT sur les RAl et les jeunes (Elloumi et al., 2020).

\subsection{Recommandations politiques: améliorer l'accès aux services techniques et d'information}

Les pépinières d'entreprises, les services d'encadrement et de conseil peuvent apporter une contribution importante au renforcement des compétences et des plans commerciaux et à concrétisation d'une idée, mais les problèmes pratiques qui demeurent doivent être résolus. Ceux-ci concernent à la fois l'accessibilité géographique de ces services et la durabilité de leurs impacts. Certains des principaux obstacles auxquels les jeunes sont confrontés en ce qui concerne l'accès à ces services techniques et d'information pourraient être surmontés en considérant les deux recommandations clés suivantes: (i) Intégrer l'incubation et l'encadrement avec d'autres services de soutien financier et 
technique; et (ii) coordonner les efforts concertés des différents acteurs et entités offrant des services d'incubation et d'encadrement.

Intégrer l'incubation et l'encadrement avec d'autres services de soutien financier et technique. Les jeunes ont généralement besoin d'un suivi après la période d'incubation, notamment d'un mentorat supplémentaire et d'un accès aux facteurs de production. En effet, les compétences et techniques acquises en agriculture risquent d'être inutiles dans la pratique si un jeune agri-entrepreneur incubé est diplômé, mais n'a toujours pas les moyens d'acheter des terres. Une innovation pilote réussie dans la transformation des aliments ne peut jamais être généralisée sans la mobilisation de ressources financières suffisantes. Par conséquent, il peut être utile d'envisager des partenariats stratégiques avec des entités privées (comme entre l'APIA et la BTS en Tunisie) ou d'intégrer des services d'incubation et d'encadrement avec des ensembles d'incitations financières.

Coordonner les efforts concertés des différents acteurs et entités offrant des services d'incubation et d'encadrement. Alors que les approches centralisées et pilotées par le gouvernement comme celles en Tunisie (Pépinières d'entreprises agricoles) et au Sénégal (MIJA) peuvent s'efforcer de garantir une couverture régionale étendue, les pays qui reposent principalement sur des initiatives privées peuvent se retrouver avec une concentration d'incubateurs dans des domaines attractifs pour ceux qui les gèrent ou les financent, ce qui ne coïncide pas nécessairement avec les priorités du pays. Cela peut conduire à une distorsion régionale, privilégiant les jeunes urbains par rapport à leurs pairs dans les zones rurales, question particulièrement importante lorsqu'il s'agit d'atténuer la migration des jeunes des campagnes vers les villes. Dans le cas où les ressources financières ne permettraient pas un réseau d'incubateurs financé par l'État, les gouvernements peuvent s'efforcer de garantir un équilibre régional par des négociations minutieuses avec les partenaires de ressources, y compris le secteur privé, et des partenariats par le biais d'une participation limitée au capital, qui peut influencer l'emplacement des incubateurs. 


\section{IMPLIQUER LES JEUNES DANS LA CONCEPTION DES POLITIQUES, DES LOIS ET DES MESURES D'INCITATION}

«Il existe de nombreux programmes et initiatives pour les jeunes, mais jamais aucun programme ni aucune initiative mise en œuvre par et avec les jeunes»Un participant à la Conférence 2019 sur l'Investissement agricole responsable pour atteindre les objectifs de développement durable d'ici $2030 .^{10}$

Il est aujourd'hui largement reconnu que «des processus d'élaboration de politiques ouverts et inclusifs contribuent à garantir que les politiques soient fondées sur de meilleures bases (OCDE, 2013)». Étant donné que «l'incidence des politiques sur différents groupes varie selon les groupes (FAO, 2002)», il est essentiel de faire participer tous ceux qui pourraient avoir un intérêt ou être touchés par de nouvelles politiques ou une réforme des politiques. Les jeunes ne sont pas uniquement des bénéficiaires passifs de programmes, projets et incitations, mais doivent plutôt être considérés comme des acteurs à part entière, les mieux placés pour connaître leurs propres forces, besoins et contraintes. II convient de noter que la priorité de la Stratégie des Nations Unies pour la jeunesse est «Engagement, participation et plaidoyer - Amplifier les voix des jeunes pour la promotion d'un monde pacifique, juste et durable (Nations Unies, 2018)». L'élaboration de politiques inclusives et participatives n'est pas une solution miracle qui garantit en soi que les jeunes aient les moyens d'investir dans des entreprises prospères. II s'agit cependant d'une condition préalable indispensable pour renforcer l'efficacité et la durabilité des interventions et mesures visant à autonomiser les jeunes agri-entrepreneurs et à rendre les zones rurales plus attractives.

Malgré un engagement de principe, des études ont conclu qu'il y a «encore un long chemin à parcourir pour garantir la participation active et concrète des jeunes dans les processus politiques (FAO, CTA et FIDA, 2014)». Notamment en raison des préjugés culturels répandus contre les jeunes dans des contextes où «l'ancienneté est [...] associée à l'autorité (FAO, CTA et FIDA, 2014)», les voix des jeunes peuvent souvent être perçues ou ignorées. Les jeunes femmes peuvent être particulièrement désavantagées, en particulier dans les milieux où la discrimination fondée sur le sexe persiste. Bien que certains documents juridiques et politiques, comme la Charte africaine de la jeunesse (Union africaine, 2006), énoncent explicitement les droits des jeunes à participer à l'élaboration des politiques, de nombreux jeunes hommes et femmes ignorent leurs droits à cet égard.

\footnotetext{
10 La Conférence sur l'augmentation des investissements responsables dans l'agriculture pour réaliser les Objectifs de développement durable à I'horizon 2030 a été organisée par la FAO à Tunis, Tunisie, les 17 et 18 octobre 2019 avec des parties prenantes de six pays - Guinée, Mali, Mauritanie, Maroc, Sénégal et Tunisie.
} 


\section{Participation des jeunes aux processus décisionnels: analyse de la situation}

Les analyses entreprises ont conduit à des conclusions similaires en considérant le cas spécifique des jeunes agri-entrepreneurs. Lorsqu'on a demandé aux participants et aux experts d'évaluer la participation des organisations de jeunesse entre 0 (pas de participation) et 5 (excellente participation), tous les pays à l'exception de la Namibie $(2,5)$ et du Mali (3) ont obtenu des scores inférieurs à 2,5. Les trois principaux défis concernent (i) le manque d'organisation et de coordination entre les jeunes dans l'agriculture et l'agro-industrie; (ii) les faibles capacités et les compétences limitées de plaidoyer des jeunes chefs de file; et (iii) le manque de mécanismes de consultation efficaces, significatifs et inclusifs dans lesquels les jeunes peuvent s'engager.

Figure 11: Participation des jeunes aux mécanismes de coordination/plateformes multipartites $(0=$ pas de participation; 5 = excellente participation)

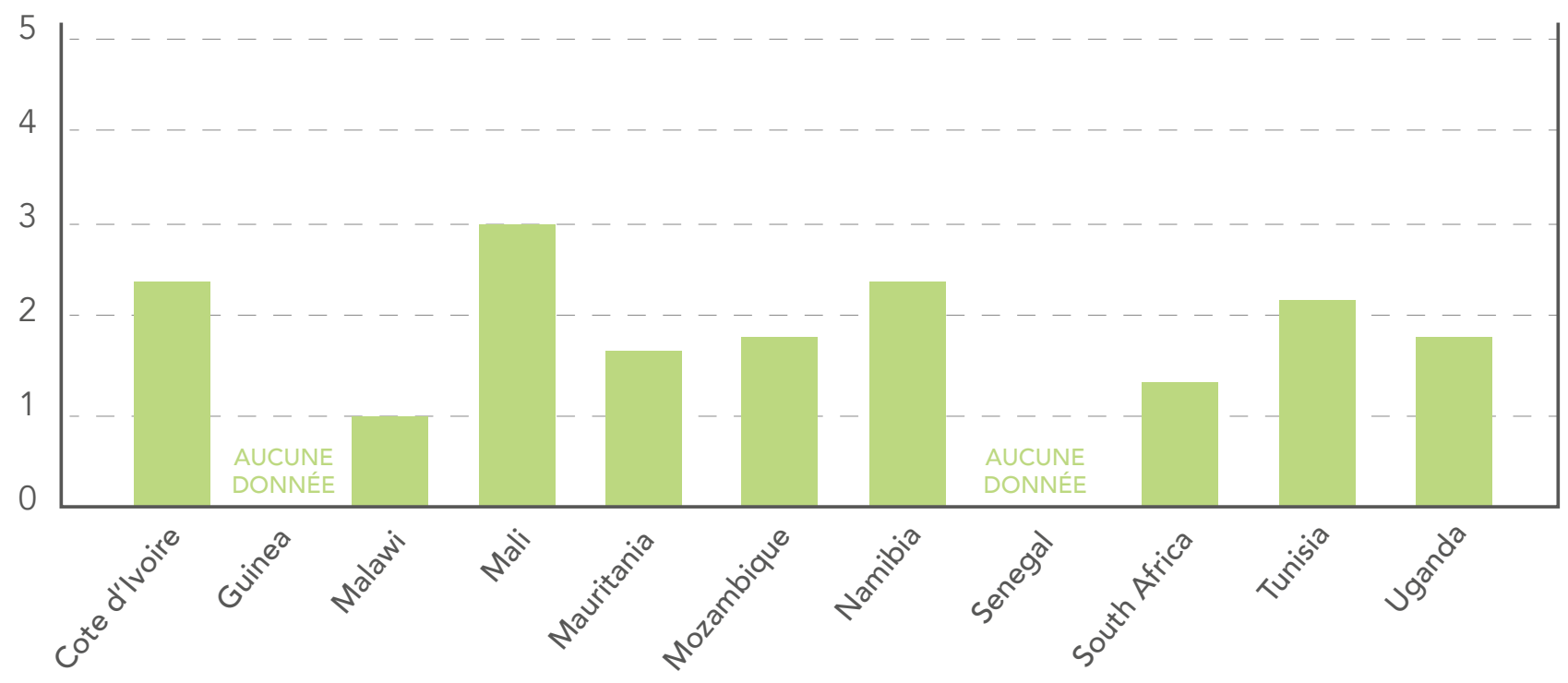

Source: Figure élaboré par l'auteur à partir de la base de données FAO RAl et des évaluations des capacités des jeunes, 2020.

Organisation et coordination. L'existence d'organisations de jeunesse bien organisées qui se coordonnent entre elles est une condition préalable à un dialogue politique significatif avec le gouvernement. En fait, les gouvernements doivent être en mesure d'identifier des interlocuteurs légitimes lors de la conception de processus d'élaboration de politiques inclusifs. La Côte d'Ivoire, le Mali, l'Afrique du Sud et le Sénégal ont des organisations indépendantes de jeunes ruraux ou de jeunes agri-entrepreneurs qui sont actives au niveau national. Au Mali, la Fédération nationale des jeunes ruraux (FENAJER) est une organisation faîtière dynamique qui regroupe plus de 5000 organisations membres (FENAJER, sans date) et est active depuis 2005. La FENAJER est elle-même une organisation membre de la Coordination nationale des organisations paysannes du 
Mali (CNOP-Mali), et une interlocutrice reconnue au Conseil supérieur de l'agriculture du Mali. En Afrique du Sud, la Chambre des jeunes de l'agriculture commerciale (CAYC) compte environ 1200 coopératives de jeunes et 1900 jeunes agriculteurs individuels. La CAYC s'est engagée assez activement dans le plaidoyer auprès des décideurs pour des politiques et des mesures d'incitation plus sensibles aux jeunes. En particulier, la CAYC a réussi à faire pression sur le Ministère de l'Agriculture et de la Réforme foncière pour établir une structure de jeunesse appelée «Jeunesse dans l'agriculture et le développement rural (YARD)», qui vise à répondre aux besoins des jeunes en mettant en œuvre des politiques et des services appropriés et l'égalité entre les jeunes, notamment par le biais du programme AgriBEE (Agricultural Black Economic Empowerment - «émancipation économique des Noirs dans l'agriculture») (Hakizimana, à paraître).

Bien que ces exemples soient encourageants, ils sont loin de constituer une norme. Dans certains pays, les organisations de jeunes ruraux et de jeunes agri-entrepreneurs opèrent à petite échelle avec de faibles capacités (comme c'est le cas dans les deux pays du Maghreb), ou il peut y avoir seulement plusieurs organisations dynamiques de taille moyenne (comme c'est le cas au Sénégal). En Ouganda, il existe de nombreuses organisations de jeunes de petite et moyenne envergure qui sont actives, mais la politisation et la «lutte» entre elles ont souvent été mentionnées comme un obstacle majeur à la participation effective des jeunes à l'élaboration des politiques par les participants à l'analyse des capacités (y compris les jeunes eux-mêmes). Dans tous les cas, le pouvoir de négociation des jeunes est considérablement réduit.

Capacités réduites et compétences de plaidoyer limitées des jeunes chefs de file. Afin de participer activement aux processus d'élaboration des politiques, les jeunes ruraux et les agri-entrepreneurs urbains ont besoin de compétences adéquates. Cependant, seuls quelques-uns d'entre eux en possèdent. Les compétences limitées de plaidoyer de leurs chefs de file sont des contraintes importantes pour de nombreuses organisations de jeunesse. Dans presque tous les pays, les gouvernements et les organisations de jeunesse ont mentionné eux-mêmes que ce manque de compétences constituait un défi majeur étant donné qu'il empêche les jeunes agri-entrepreneurs de défendre efficacement leurs intérêts face à d'autres parties prenantes bien informées et équipées, telles que les secteurs privés à grande échelle.

Mécanismes de consultation et les différentes formes du dialogue politique. Une autre question importante est le cadre de gouvernance institutionnelle. Les mécanismes de consultation formels, tels que les plates-formes multi-acteurs, peuvent fournir aux organisations de jeunesse l'occasion de s'engager avec les décideurs politiques. Au Mali, par exemple, le Conseil supérieur de l'agriculture a un mandat très large, qui comprend notamment le suivi de la mise en œuvre de la loi d'orientation agricole du pays et l'élaboration de propositions relatives à la loi d'orientation. FENAJER participe activement au Conseil. Dans d'autres cas, de tels mécanismes peuvent exister, mais ils peuvent avoir moins de pouvoir sur les processus de prise de décision politique. Dans d'autres cas encore, ils peuvent ne pas exister du tout. Les jeunes moins organisés sont plus pénalisés que ceux des pays où il existe un haut degré d'organisation et de coordination (comme au Mali et en Afrique du Sud). 


\section{Recommandations: permettre aux jeunes de participer à l'élaboration des politiques}

Même si les jeunes eux-mêmes ont la responsabilité ultime de faire entendre leur voix, les gouvernements peuvent apporter une contribution importante à l'autonomisation des jeunes afin qu'ils s'engagent dans les processus d'élaboration des politiques en (i) soutenant les initiatives de renforcement des capacités ciblant les organisations de jeunesse; (ii) formalisant le dialogue politique à travers des plateformes multi-acteurs; et (iii) en communiquant activement l'existence, la nature et les objectifs de ces plates-formes.

Soutenir les initiatives de renforcement des capacités ciblant les organisations de jeunesse. Lorsque les organisations de jeunesse sont faibles ou inexistantes, les gouvernements peuvent souhaiter rechercher le soutien de tiers indépendants (ONG, organisations internationales ou coopération bilatérale au développement) pour fournir un soutien au développement des capacités. Des interventions ciblées pourraient viser à renforcer les capacités organisationnelles des organisations de jeunesse, les compétences de plaidoyer des chefs de file de la jeunesse, ainsi que les compétences techniques sur certains sujets tels que l'investissement responsable ou le développement rural.

Formaliser le dialogue politique et créer des plates-formes multi-acteurs. Les platesformes multipartites efficaces et inclusives offrent aux organisations de jeunesse un espace au sein duquel elles peuvent communiquer leurs besoins et leurs priorités et s'engager directement avec les décideurs. Finalement, un dialogue institutionnalisé avec les organisations de jeunesse sera également bénéfique pour les gouvernements: les jeunes eux-mêmes sont les mieux placés pour connaître leurs forces, leurs besoins et leurs contraintes. Connaître leurs besoins permettra aux décideurs de concevoir des programmes d'incitation et des mécanismes de soutien plus efficaces.

Communiquer l'existence, la nature et les objectifs des plateformes multi-acteurs en utilisant tous les canaux de communication possibles (radio, affiches et réseaux sociaux, entre autres) pour sensibiliser les organisations de jeunesse aux opportunités de dialogue politique. En outre, il est important d'expliquer clairement comment les organisations peuvent présenter leurs demandes d'adhésion ou participer à d'autres formes d'association (par exemple, le statut d'observateur).

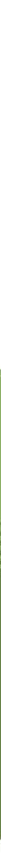




\section{CINQ RECOMMANDATIONS POLITIQUES POUR STIMULER ET PÉRENNISER LES INVESTISSEMENTS DES JEUNES AGRI-ENTREPRENEURS DANS L'AGRICULTURE ET LES SYSTÈMES ALIMENTAIRES}

Les incitations ciblées sont essentielles pour permettre aux jeunes d'investir dans l'agriculture et les systèmes alimentaires. Dans un contexte de marchés de capitaux imparfaits, la fourniture d'incitations financières tant aux jeunes agri-entrepreneurs (prêts directs et subventions) qu'aux institutions financières (à travers des fonds de garantie) est indispensable dans de nombreux pays. Bien que l'importance des incitations financières soit indéniable, les jeunes agri-entrepreneurs ont généralement besoin d'un soutien supplémentaire pour créer et développer des entreprises florissantes. Cette panoplie d'instruments inclut les incubateurs d'entreprises, l'encadrement et d'autres services de conseil qui aident les jeunes à développer, piloter et mettre en œuvre des projets économiquement viables. En outre, il est essentiel que les jeunes participent activement à tous les processus d'élaboration des politiques qui les concernent afin de garantir que les mesures politiques et les incitations seront efficaces et généreront des retours sur investissement élevés.

Afin de maximiser les effets positifs des incitations à l'investissement sur les jeunes, les cinq recommandations clés suivantes devraient être prises en compte: (i) élaborer des critères de ciblage clairs qui déterminent les conditions d'accès aux programmes d'incitation; (ii) fournir des ensembles d'incitations plutôt que des interventions de soutien autonomes ou isolées; (iii) veiller à ce que la politique globale et le cadre juridique favorisent plutôt qu'entravent les investissements des jeunes agri-entrepreneurs; (iv) développer une stratégie de communication adaptée aux jeunes et adaptée au contexte; et (v) faire participer les jeunes aux processus d'élaboration des politiques.

\section{(i) Développer des critères de ciblage clairs qui déterminent les conditions d'accès aux programmes d'incitation.}

Les agences fournissant des incitations financières, des services d'incubation ou un accès à la terre devraient évaluer soigneusement dans quel but ces incitations à l'investissement financier sont nécessaires (par exemple, pour impliquer les jeunes titulaires d'un diplôme universitaire en agriculture, pour améliorer la durabilité des modèles de production, etc.). En conséquence, des critères de ciblage spécifiques qui définissent qui devrait bénéficier 
de ces incitations doivent être établis. Si l'autonomisation des jeunes est l'objectif principal, des critères spécifiques à l'âge doivent être établis pour garantir que l'incitation est spécifique aux jeunes. Si les capacités d'une certaine catégorie de jeunes (comme les diplômés) doivent être mises à profit, ou si un groupe particulièrement défavorisé (comme les jeunes femmes des zones rurales reculées) doit être autonomisé, cela devra également être indiqué dans les critères de ciblage. Enfin, si l'autonomisation des jeunes est un objectif important, mais pas le seul, il faut s'assurer que les critères du programme d'incitation favorisent l'accès des jeunes plutôt qu'ils ne les ignorent par inadvertance.

\section{(ii) Fournir des ensembles d'incitations plutôt que des interventions de soutien autonomes ou isolées.}

Même s'il semble utile d'offrir différents types d'incitations à autant de jeunes que possible, il peut être préférable de proposer des ensembles d'incitations à un plus petit nombre de bénéficiaires en cas de budgets limités. En combinant des prêts avec des subventions d'investissement, des services d'incubation, d'encadrement et de mentorat, les chances d'avoir un retour sur investissement durable chez les jeunes peuvent être beaucoup plus élevées qu'en consacrant des efforts isolés et dispersés. Dans certains cas, lorsque l'on considère les économies les moins avancées, il faut également reconnaître que des interventions de développement rural plus étendues peuvent être nécessaires pour autonomiser les jeunes des zones rurales (FIDA, 2019). Une action concertée entre les agences, y compris les bailleurs de fonds, le secteur privé et les institutions philanthropiques est essentielle à cet égard.(iii) Ensure that the overall policy and legal framework empowers, rather than impedes young agri-entrepreneurs' investments.

(iii) Veiller à ce que la politique globale et le cadre juridique renforcent les investissements des jeunes agri-entrepreneurs au lieu de les entraver.

Même lorsque des systèmes d'incitation cohérents et bien structurés sont en place, leur efficacité peut être limitée en l'absence d'une politique globale et d'un cadre juridique qui donnent les moyens nécessaires aux jeunes agri-entrepreneurs. Les interventions qui visent à améliorer les réglementations favorables au secteur financier, les politiques et lois foncières sensibles aux jeunes, ainsi que les cadres juridiques et réglementaires qui favorisent l'étiquetage et la commercialisation des denrées alimentaires de qualité sont quelques-unes des mesures que les décideurs peuvent prendre pour aligner les cadres politiques et juridiques sur une volonté largement exprimée en faveur des jeunes.

(iv) Développer une stratégie de communication adaptée aux jeunes et spécifique au contexte.

Il est illusoire de supposer qu'une masse critique de jeunes — en particulier ceux des zones rurales - lira et comprendra le journal officiel du gouvernement ou d'autres sources officielles de communication pour connaître les nouvelles mesures d'incitation, opportunités d'investissement et possibilités de s'engager dans les processus d'élaboration des politiques. La communication doit être sensible aux jeunes et spécifique au contexte, en utilisant Internet, les réseaux sociaux, les radios, la télévision, des affiches et d'autres formats, le cas échéant. Les jeunes eux-mêmes sont les mieux placés pour connaître leurs modes de communication préférés. 
Cinq recommandations politiques pour stimuler et pérenniser les investissements des jeunes agri-entrepreneurs dans l'agriculture et les systèmes alimentaires

(v) Faire participer les jeunes aux processus d'élaboration des politiques.

Dialoguer avec les organisations de jeunesse et d'autres parties prenantes concernées dans les processus d'élaboration des politiques, notamment dans la conception de programmes d'incitation à l'investissement, plutôt que de les considérer uniquement comme des bénéficiaires passifs. Les jeunes eux-mêmes sont les mieux placés pour connaître leurs forces, leurs besoins et leurs difficultés. Une attention particulière devra être accordée au fait de s'assurer que les voix des groupes éventuellement marginalisés soient entendues en garantissant leur participation significative et inclusive. Ultérieurement, les jeunes devraient être directement impliqués dans la définition des critères de performance et participer au suivi de l'impact des programmes d'incitation. Lorsque les organisations de jeunesse manquent de capacités pour remplir ce rôle ou si le nombre de petites organisations les empêche de fusionner en raison de leur fragmentation, il est conseillable d'envisager la possibilité de soutenir un tiers neutre (comme une ONG) qui renforcera leurs capacités.

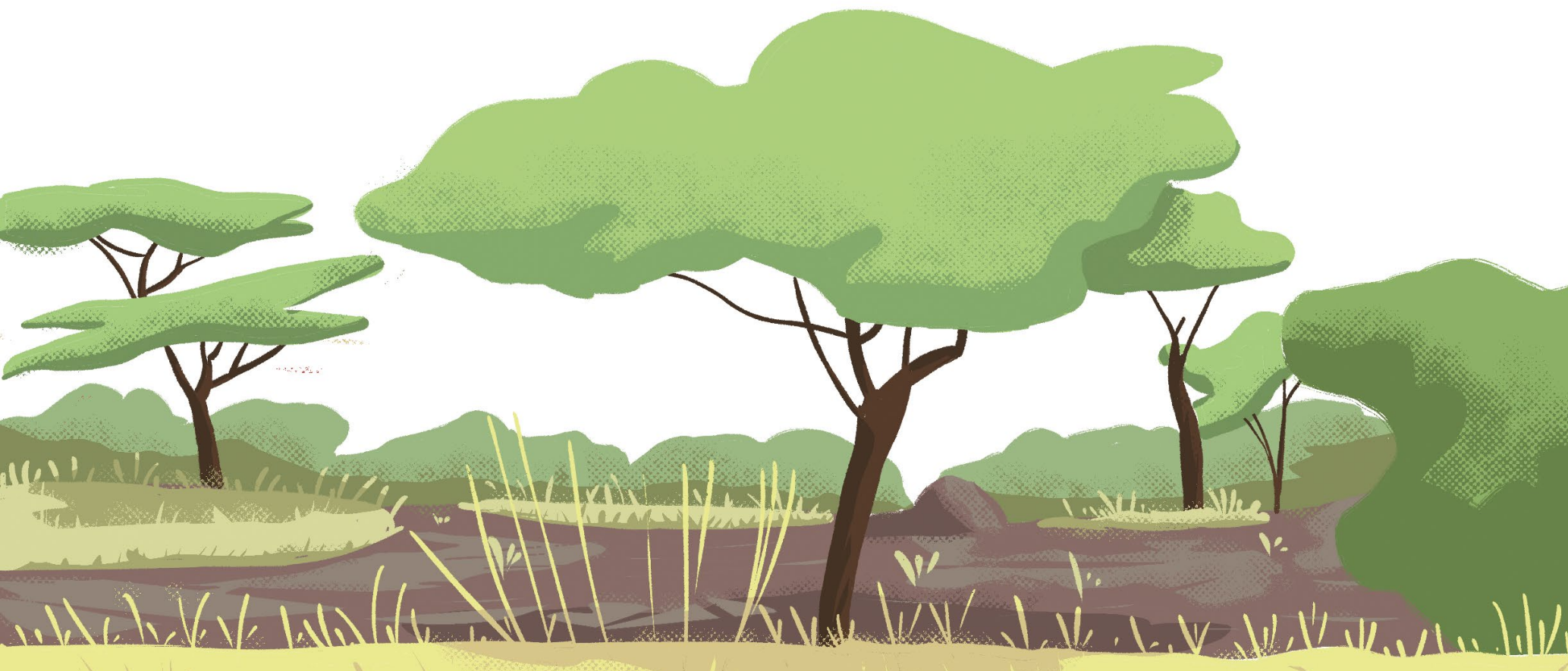





\section{Observations finales et perspectives}

Ce rapport a résumé les enseignements tirés des travaux d'évaluation et de planification stratégique de la FAO avec onze pays africains dans le but de permettre aux jeunes d'investir dans l'agriculture et les systèmes alimentaires. II a par ailleurs fourni une série de recommandations aux décideurs politiques qui visent à soutenir des systèmes d'incitation à l'investissement plus efficaces, inclusifs et axés sur les jeunes.

Si les évaluations effectuées à ce jour ont contribué à la production d'un ensemble significatif de données empiriques, il est essentiel d'effectuer des analyses approfondies sur des sujets spécifiques pour mieux comprendre l'utilité de certains types d'incitations. En particulier, une évaluation comparative de la performance des systèmes d'incitation dans différents pays contribuera certainement à l'identification des bonnes pratiques qui fonctionnent. II faut également reconnaître que ce rapport a utilisé un échantillon de pays ayant une portée régionale limitée. Même si les onze pays peuvent être assez représentatifs d'un contexte africain plus large, couvrant le Maghreb, ainsi que l'Afrique orientale, occidentale et australe dans sa complexité, l'applicabilité des résultats dans d'autres régions, comme l'Asie ou I'Amérique latine et les Caraïbes, devra faire l'objet d'un examen critique. À cet égard, les efforts récents d'AsiaDHRRA pour appliquer le R-CAT en Asie du Sud-Est (AsiaDHRRA, 2020) doivent être appréciés ainsi que les résultats obtenus dans cette région du monde comparés à ceux présentés dans ce rapport.

D'autre part, un soutien supplémentaire est nécessaire pour passer de l'évaluation et de la planification à la mise en œuvre des recommandations. Cela nécessite d'une part l'élaboration d'orientations normatives. Le guide de la FAO et du Centre de l'Université de Columbia sur les investissements durables (CCSI) sur les incitations à l'investissement responsable dans l'agriculture et les systèmes alimentaires, actuellement en cours de préparation, apportera une contribution utile à cet égard. D'autre part, un soutien de suivi est nécessaire au niveau des pays. Les modèles innovants, tels que la mise en place d'un groupe de réflexion en Tunisie chargé de proposer des solutions réalisables pour autonomiser les jeunes agrientrepreneurs qui seraient mises à l'essai, devraient idéalement être généralisés et étendus à d'autres pays.

Enfin, le partage des connaissances et l'engagement continu entre les principales parties prenantes au niveau régional doivent être encouragés et renforcés. L'expérience réussie de la Conférence 2019 sur l'investissement agricole responsable pour atteindre les Objectifs de développement durable d'ici 2030 avec six pays francophones pourrait constituer un point de référence utile à cet égard. 


\section{Références}

- ADIE. sans date. Obtenir une garantie du fonds de garantie d'investissements prioritaires (FONGIP) par le biais d'établissements bancaires [en ligne]. [Cité 21 septembre 2020]. http://www.servicepublic.gouv.sn/index.php/demarche_administrative/demarche/1/1189

- African Union. 2006. African Youth Charter. https://www.un.org/en/africa/osaa/pdf/au/african_youth_charter_2006.pdf

- Alexandratos, N. \& Bruinsma, L. 2012. World agriculture towards 2030/2050: the 2012 revision. FAO ESA Working Paper, 12-03.

- Amadhila, A. non publié. Key challenges that need to be addressed to create an enabling environment that empowers young agri-entrepreneurs to carry out responsible investment in agriculture and food systems in Namibia. Study commissioned by FAO and ITOCA.

- APIA. 2020. Quels sont les services que peut offrir une pépinière. In: APIA [en ligne]. [Cité 21 septembre 2020].

http://www.apia.com.tn/qu-elles-sont-les-services-que-peut-offrir-une-pep.html

- APIEX Moçambique. 2018. Investment Guide for Mozambique: Agroprocessing and light manufacturing sectors. www.intracen.org/uploadedFiles/intracenorg/Content/Redesign/ Projects/PIGA/PIGA_Mozambique_ENG_20180613_img_web_pages.pdf

- AsiaDHRRA. 2020. Engaging ASEAN Youth on Responsible Agriculture Investments. http://asiadhrra.org/wordpress/wp-content/uploads/2020/06/CFS-RAI-Monograph.pdf

- Bank of Namibia Research Department. 2004. Promoting microfinance activities in Namibia: a regulatory and supervisory perspective. Working Paper of the Bank of Namibia. (en ligne: https://www.bon.com.na/CMSTemplates/Bon/Files/bon.com.na/28/2854be4fd3a3-4d93-98d2-e7527965a411.pdf).

- Bellu, L. G., Mueller, M. \& Kavallari, A. 2015. Achieving Zero Hunger: The Critical role of investments in social protection and agriculture. Rome, Italie, FAO. 39 pp. (en ligne: http://www.fao.org/documents/card/en/c/91014696-3723-4df5-b729-2b4e55b22e8f/).

- CFS. 2014. Principles for Responsible Investment in Agriculture and Food Systems. CFS. http://www.fao.org/3/a-au866e.pdf

- Comité technique foncier et développement. 2020. La question de l'accès des jeunes à la terre: Éléments pour mieux concevoir et suivre les interventions et les politiques de développement rural dans la durée. (en ligne: http://www.foncier-developpement.fr/wpcontent/uploads/2020_CTFD_Lacc\%C3\%A8s-des-jeunes-\%C3\%A0-la-terre-VF-online.pdf).

- Cornerstone Economic Research (Pty) Ltd. 2014. Micro Agricultural Financial Institutions of South Africa. http://www.gtac.gov.za/programmes-and-services/ public-expenditure-and-policy-analysis 
- Côte d'Ivoire. 2017. Programme national d'investissement agricole de deuxième génération. http://extwprlegs1.fao.org/docs/pdf/IVC176030.pdf

- De La O Campos, A.P., Villani, C., Davis, B. \& Takagi, M. 2018. Ending extreme poverty in rural areas - Sustaining livelihoods to leave no one behind. Rome, Italie, FAO. 85 pp. (en ligne: http://www.fao.org/publications/card/en/c/CA1908EN/).

- Elloumi, M., Fiedler, Y., Ben Saad, A., Ouertani, E., Yangui, A. \& Labidi, A. 2020. Pour un environnement institutionnel et financier favorable à l'investissement par les jeunes dans I'agriculture et les systèmes alimentaires en Tunisie: Document d'orientation. Rome, Italy, FAO and INRAT. 16 pp. (en ligne: http://www.fao.org/documents/card/fr/c/cb0884fr/).

- Eiselen, B. et Fiedler, Y. 2020. Stimuler et pérenniser les investissements des jeunes agrientrepreneurs - Outil de planification stratégique. Rome, FAO et HAFL (en ligne: https://doi.org/10.4060/cb1367fr).

- eMJee Consult. 2018. Final evaluation of the SNV Opportunities for Youth Employment (OYE) project in Tanzania, Rwanda and Mozambique. https://snv.org/cms/sites/default/ files/explore/download/final_evaluation_report.pdf

- European Commission. 2002. Benchmarking of Business Incubators - Final Report. http://ec.europa.eu/DocsRoom/documents/2769/attachments/1/translations/en/renditions/pdf

- FAO. 2002. Participatory Policy Reform from a Sustainable Livelihoods Perspective. Rome, FAO. (en ligne: http://www.fao.org/3/ad688e05.htm).

- FAO. 2012a. The State of Food and Agriculture 2012: Investing in agriculture for a better future. Rome, FAO. (en ligne: http://www.fao.org/publications/sofa/2012/en/).

- FAO. 2012b. FAO approaches to capacity development in programming: processes and tools. Rome, FAO. (en ligne: http://www.fao.org/fileadmin/user_upload/capacity_ building/FAO_CD_LM2.pdf).

- FAO. 2012 c. Voluntary Guidelines on the Responsible Governance of Tenure of Land, Fisheries and Forests in the Context of National Food Security. Rome, FAO. (en ligne: http://www.fao.org/3/i2801e/i2801e.pdf).

- FAO. 2014. Contribution to the 2014 United Nations Economic and Social Council (ECOSOC) Integration Segment

- FAO. 2017. Ending poverty and hunger by investing in agriculture and rural areas. Rome, FAO. (en ligne: http://www.fao.org/policy-support/tools-and-publications/ resources-details/en/c/1027619/).

- FAO. 2018a. Credit to agriculture [en ligne]. [Cité 21 septembre 2020]. http://www.fao.org/economic/ess/investment/credit/en/

- FAO. 2018b. Empowering youth to engage in responsible investment in agriculture and food systems: Rapid capacity assessment tool. Rome, Italie, FAO. 30 pp. (en ligne: http://www.fao.org/publications/card/en/c/CA2061EN). 
- FAO. 2018 c. Empowering youth to engage in responsible investment in agriculture and food systems: Guidance for organizers and facilitators of the rapid capacity assessment tool. Rome, Italie, FAO. 44 pp.

(en ligne: http://www.fao.org/publications/card/en/c/CA2065EN/).

- FAO. 2018d. Strengthening sustainable food systems through geographical indications: An analysis of economic impacts. FAO Investment Centre. Directions in Investment. Rome, Italie, FAO. 135 pp. (en ligne: http://www.fao.org/publications/card/en/c/l8737EN/).

- FAO. 2019. Empowering youth to engage in responsible investment in agriculture and food systems: Challenges, opportunities and lessons learned from six African countries (Côte d'Ivoire, Malawi, Mozambique, Namibia, South Africa and Uganda). Rome, Italie, FAO. 40 pp. (en ligne: http://www.fao.org/documents/card/en/c/CA2877EN).

- FAO. 2020. COVID-19 and the risk to food supply chains: How to respond? Rome, Italie, FAO. 7 pp. (en ligne: http://www.fao.org/documents/card/en/c/ca8388en).

- FAO, CTA \& IFAD. 2014. Youth and agriculture: Key challenges and concrete solutions. Rome, FAO. (en ligne:http://www.fao.org/3/a-i3947e.pdf).

- FAO et INRAT. 2020a. Dynamique de l'investissement dans le système agricole tunisien et perspectives de développement des investissements par et pour les jeunes. Rome, Italie, FAO. 88 pp. (en ligne: http://www.fao.org/documents/card/en/c/cb0563fr).

- FAO et INRAT. 2020b. Promouvoir et pérenniser les investissements des jeunes agrientrepreneurs en Tunisie: Rapport final du projet - Investissements responsables par et pour les jeunes dans l'agriculture et les systèmes alimentaires en Tunisie. Tunis, Tunisie, FAO. 48 pp. (en ligne: http://www.fao.org/documents/card/en/c/cb0943fr).

- FAO, IFAD, UNICEF, WFP \& WHO. 2020. The State of Food Security and Nutrition in the World 2020: Transforming food systems for affordable healthy diets. The State of Food Security and Nutrition in the World (SOFI) No. 2020. Rome, Italy, FAO, IFAD, UNICEF, WFP and WHO. 320 pp. (en ligne: http://www.fao.org/documents/card/en/c/ca9692en).

- FENAJER. sans date. About [en ligne]. http://www.fenajer-mali.org/about/

- Fernández Fernández, M. T., Blanco Jiménez, F.J. \& Cuadrado Roura, J. R. 2015. Business incubation: innovative services in an entrepreneurship ecosystem. The Service Industries Journal, 35(14): 783-800.

- Fiedler, Y. \& lafrate, M. 2016. Trends in Foreign Direct Investment in Food, Beverages and Tobacco. FAO Commodity and Trade Policy Research Working Paper. Rome, Italie, FAO. 46 pp. (en ligne: http://www.fao.org/documents/card/ fr/c/8d0a32e7-e7b0-4ad8-94bb-9a4d19aacd71/).

- FONGIP. sans date. Garantie individuelle - Fongip [en ligne]. [Cité 21 septembre 2020]. http://www.fongip.sn/garantie-individuelle/ 
- Forbes Afrique. 2016. Saboutech, premier incubateur spécialisé de Guinée. In: Forbes Afrique. [en ligne]. [Cité 21 septembre 2020]. https://forbesafrique.com/ saboutech-premier-incubateur-specialise-de-guinee/

- Hakizimana, C. forthcoming. Responsible investments in agriculture and food systems in South Africa: Challenges and Opportunities for young agri-entrepreneurs in a Changing Policy Environment. Rome, FAO.

- IFAD. 2011. Viewpoint - Smallholders can feed the world. Rome, IFAD. (also available at https://www.ifad.org/documents/38714170/40706188/Smallholders+can+feed+the+ world_e.pdf/460ca6c2-7621-40d8-9f79-a56f6f8fa75e).

- IFAD. 2019. Rural development report: Creating opportunities for rural youth. Rome, IFAD. (en ligne: https://www.ifad.org/documents/38714170/41133075/RDR_report. pdf/7282db66-2d67-b514-d004-5ec25d9729a0).

-ILO. 2013. Guide to the millennium development goals employment indicators. Geneva, ILO.

- ILO. 2014. Is education the solution to decent work for youth in developing countries? Geneva, ILO.

-ILO. 2017. Decent Jobs for Youth (4): Boosting decent jobs for youth, greening the economy. ILO.

https://www.decentjobsforyouth.org/wordpress/wp-content/uploads/2017/08/ThematicPlan-4-Green-Jobs.pdf

- ILO. 2020. Global Employment Trends for Youth 2020: Technology and the future of jobs. Geneva, ILO. (en ligne: http://www.ilo.org/global/publications/books/WCMS_737648/ lang--en/index.htm).

- Journal officiel de la République du Mali. 2017. Loi n²017-001 du 11 avril 2017 portant sur le foncier agricole, art. 13.

- Journal officiel de la République tunisienne. 2019. Loi nº 2019-47 du 29 mai 2019, relative à l'amélioration du climat de l'investissement.

- NDI \& CPGD. 2016. Youth's Concerns and their Expectations for Socio-economic Development in Mozambique: Findings from Focus Group Discussions. https://www.ndi.org/sites/default/files/YouthNDIReport_FINAL_11March\%202016.pdf

- OECD. 2013. Government at a Glance 2013. Paris, OECD Publishing.

- OECD. 2018. Making Blended Finance Work for the Sustainable Development Goals. Paris, OECD Publishing. 176 pp. (en ligne: https://doi.org/10.1787/9789264288768-en.).

- Rapsomanikis, G. 2015. The economic lives of smallholder farmers. Rome, Italie, FAO. 48 pp. (en ligne: http://www.fao.org/documents/card/en/c/b09e4a66-/). 
- Schmidhuber, J. \& Qiao, B. 2020. Comparing crises: Great Lockdown versus Great Recession. Rome, Italie, FAO. 40 pp.

(en ligne: http://www.fao.org/documents/card/en/c/ca8833en).

- Sénégal - Ministère de l'Économie, des Finances et du Plan. sans date. Introduction au débat d'orientation budgétaire 2017. Document de programmation budgétaire et économique pluriannuelle (DPBEP) 2018-2020.

- South Africa. 2020. Government Gazette, 3 January 2020, No. 42939. https://www.gov.za/sites/default/files/gcis_document/202001/42939gon2.pdf

- Tavares, A.T., Toledano, P., Johnson, L. \& Sachs, L.E. 2017. Rethinking investment incentives: trends and policy options.

(en ligne: https://doi.org/10.7312/columbia/9780231172981.001.0001).

- Tavares-Lehmann, A.T. 2017. Types of investment incentives. Rethinking investment incentives: trends and policy options, pp. 17-44.

(en ligne https://doi.org/10.7312/columbia/9780231172981.001.0001).

- Turvey, C. 2017. Inclusive finance and inclusive rural transformation. IFAD Research Series, 10. (en ligne: https://www.ifad.org/documents/38714170/39318394/ Research+Series+Issue+10+-+Inclusive+finance+and+inclusive+rural+transformation. pdf/d4958022-b05a-40ff-aff0-4d236b7ca3eb?eloutlink=imf2ifad).

- UNCTAD. 2003. World investment report 2003. FDI policies for development : national and international perspectives: overview. New York, United Nations.

- UNCTAD. 2014. World Investment Report 2014. Investing in the SDGs: an action plan. 265 pp.

- UNCTAD. 2020. World Investment Report 2020 - International Production Beyond the Pandemic. New York, United Nations. (en ligne: https://unctad.org/en/pages/ PublicationWebflyer.aspx?publicationid=2769).

- UNDP. 2015. Guidance Note - A Development Approach to Migration and Displacement. UNDP. [Cité 21 septembre 2020]. https://www.undp.org/content/undp/en/home/ librarypage/poverty-reduction/guidance-note---migration-and-displacement.html

- United Nations. 2013. World Youth Report 2013: Youth and Migration. New York, United Nations. (en ligne: https://doi.org/10.18356/66b5c30f-en.).

- United Nations. 2018. United Nations Youth Strategy. Youth 2030 - working with and for the youth. https://www.un.org/youthenvoy/wp-content/uploads/2018/09/18-00080_UNYouth-Strategy_Web.pdf

- United Nations. 2019. Youth (thematic website) [en ligne]. [Cité 21 septembre 2020]. https://www.un.org/en/sections/issues-depth/youth-0/index.html 
-World Bank. 2020a. Poverty - Overview. In: World Bank [en ligne]. [Cité 21 septembre 2020]. https://www.worldbank.org/en/topic/poverty/overview

-World Bank. 2020b. Updated estimates of the impact of COVID-19 on global poverty [en ligne]. [Cité 21 septembre 2020]. https://blogs.worldbank.org/opendata/ updated-estimates-impact-covid-19-global-poverty

- YPARD. 2017. 9 ways to engage youth in agriculture [en ligne]. [Cité 21 septembre 2020]. https://ypard.net/news/9-ways-engage-youth-agriculture59 




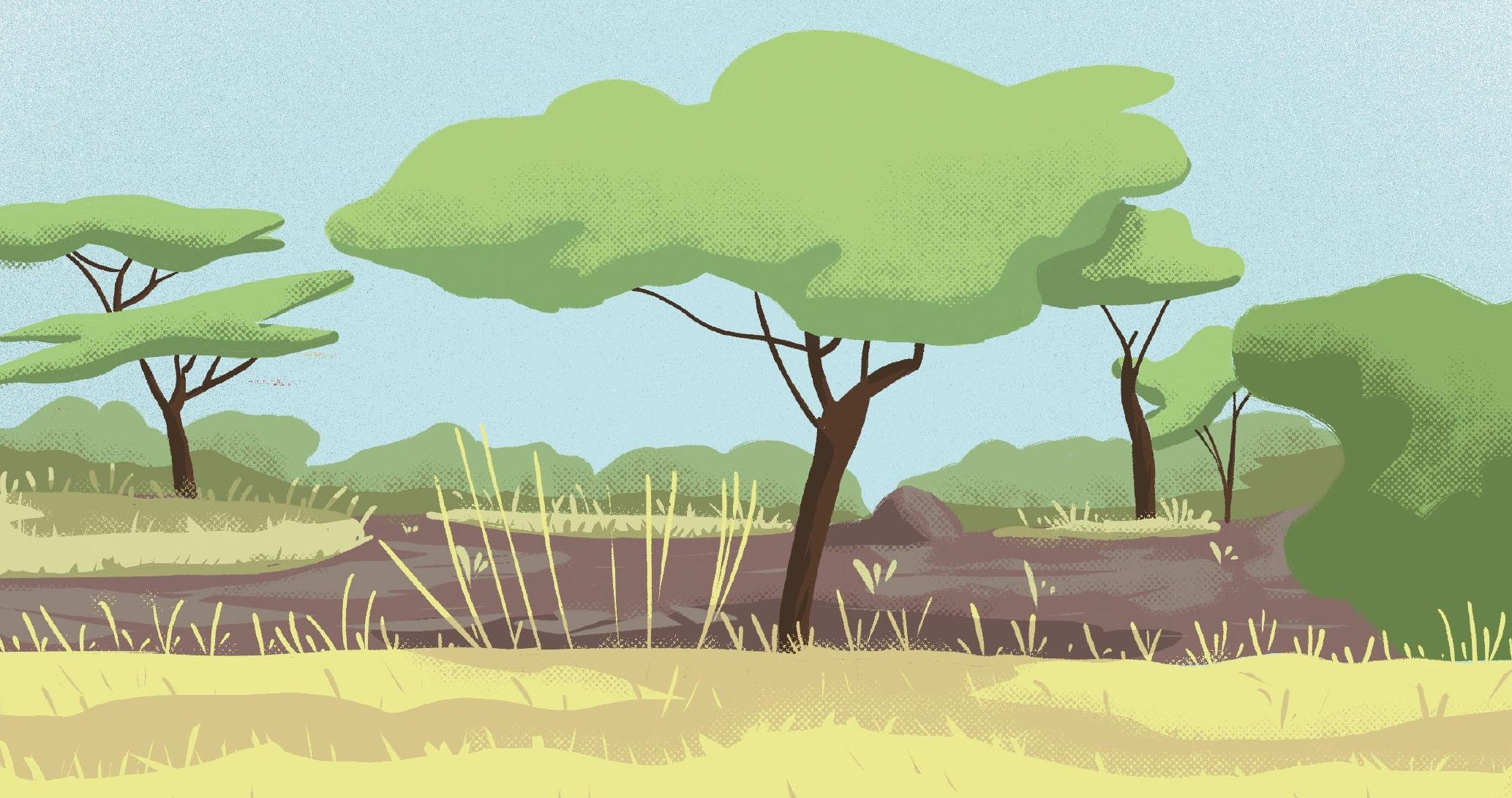

Avec le soutien de:

T. Schweizerische Eidgenossenschaft

Confédération suisse

Confederazione Svizzera

Confederaziun svizra
Département fédéral de l'économie,

de la formation et de la recherche DEFR

Office fédéral de l'agriculture OFAG 